\title{
The interface between assisted reproductive technologies and genetics: technical, social, ethical and legal issues
}

Sirpa Soini $^{1,2}$, Dolores Ibarreta ${ }^{\dagger, 3}$, Violetta Anastasiadou ${ }^{4}$, Ségolène Aymé ${ }^{5}$, Suzanne Braga ${ }^{6}$, Martina Cornel ${ }^{7}$, Domenico A. Coviello ${ }^{8}$, Gerry Evers-Kiebooms ${ }^{9}$, Joep Geraedts ${ }^{10}$, Luca Gianaroli ${ }^{11}$, Joyce Harper ${ }^{12}$, György Kosztolanyi ${ }^{13}$, Kersti Lundin ${ }^{14}$, Emilio Rodrigues-Cerezo ${ }^{\dagger, 3}$, Karen Sermon ${ }^{15}$, Jorge Sequeiros ${ }^{16}$, Lisbeth Tranebjaerg ${ }^{17}$ and Helena Kääriäinen ${ }^{*} 18$ on behalf of ESHG and ESHRE

${ }^{1}$ Department of Medical Genetics, University of Turku, Turku, Finland; ${ }^{2}$ Faculty of Law, Medical and Biolaw, University of Helsinki, Helsinki, Finland; ${ }^{3}$ European Commission's Joint Research Centre (Institute of Prospective Technological Studies), Seville, Spain; ${ }^{4}$ Archbishop Hospital and Cyprus Institute of Neurology and Genetics, Nicosia, Cyprus; ${ }^{5}$ INSERM SC11, Paris, France; ${ }^{6}$ SGMG/SSGM, Bern, Switzerland; ${ }^{7}$ VU University Medical Centre, Amsterdam, The Netherlands; ${ }^{8}$ Laboratorio di Genetica Medica, Ospedale Maggiore Policlinico, Mangiagalli e Regina Elena, Milano, Italy; ${ }^{9}$ Psychosocial Genetics Unit, Department of Human Genetics, University of Leuven, Leuven, Belgium;

${ }^{10}$ Department of Clinical Genetics, University Hospital Maastricht, Maastricht, The Netherlands; ${ }^{11}$ SISMeR, Centre for Reproductive Medicine, Bologna, Italy; ${ }^{12} U C L$ Centre for PGD, London, UK; ${ }^{13}$ Department of Medical Genetics, University of Pécs, Pécs, Hungary; ${ }^{14}$ SU/Sahlgrenska, Göteborg, Sweden; ${ }^{15}$ Centre for Medical Genetics, Dutch-speaking Brussels Free University (Vrije Universiteit Brussel, VUB), Brussels, Belgium; ${ }^{16}$ Department of Human Genetics, University of Porto, Porto, Portugal; ${ }^{17}$ Department of Audiology, H:S Bispebjerg Hospital and Wilhem Johannsen Centre of Functional Genomics, University of Copenhagen, Copenhagen, Denmark; ${ }^{18}$ Department of Medical Genetics, University of Turku, Turku, Finland

The interface between assisted reproductive technologies (ART) and genetics comprises several sensitive and important issues that affect infertile couples, families with severe genetic diseases, potential children, professionals in ART and genetics, health care, researchers and the society in general. Genetic causes have a considerable involvement in infertility. Genetic conditions may also be transmitted to the offspring and hence create transgenerational infertility or other serious health problems. Several studies also suggest a slightly elevated risk of birth defects in children born following ART. Preimplantation genetic diagnosis (PGD) has become widely practiced throughout the world for various medical indications, but its limits are being debated. The attitudes towards ART and PGD vary substantially within Europe. The purpose of the present paper was to outline a framework for development of guidelines to be issued jointly by European Society of Human Genetics and European Society of Human Reproduction and Embryology for the interface between genetics and ART. Technical, social, ethical and legal issues of ART and genetics will be reviewed. European Journal of Human Genetics (2006) 14, 588-645. doi:10.1038/sj.ejhg.5201598

Keywords: assisted reproductive technology; birth defects; counselling; genetic diseases; infertility; preimplantation genetic diagnosis

${ }^{*}$ Correspondence: Professor H Kääriäinen, Department of Medical Genetics, University of Turku, Kiinamyllykatu 10, FIN-20520 Turku, Finland. E-mail: hkaaria@utu.fi

Disclaimer: The views expressed in this study do not necessarily reflect those of the European Commission (EC). 


\section{Introduction}

Nearly one million babies have been born worldwide as the result of assisted reproductive techniques (ART) since the birth of the first baby conceived using in vitro fertilisation (IVF) techniques in $1978 .{ }^{1}$

Infertility is regarded as a health problem ${ }^{2}$ and affects approximately every 6th couple in the Western countries. It is not, however, regarded as a public health issue in every country. ${ }^{3}$ The number of infertile couples is increasing, not least because of advanced maternal age. Also, the quality of gametes has deteriorated, for instance, owing to lifestyle habits and environmental factors.

The goal of infertility services (counselling, diagnostics, treatments) is to help people with fertility problems or genetic conditions by finding solutions to their reproductive plans, for example, by ART, which is now important also for normally fertile couples whose children might inherit a serious genetic disease or even for those who wish to save a sibling's life. Counselling is an essential part of all the treatments.

Genetic causes have a considerable involvement in infertility. Well-known examples are some chromosomal translocations or sex-chromosomal abnormalities and Ychromosome deletions. Advanced maternal age has increased sporadic chromosomal anomalies with conception. Many of the reasons and their nature are, nevertheless, this far unknown. Genetic conditions may be transmitted to the offspring and hence create transgenerational infertility or other serious health problems. The use of donated gametes and embryos needs attention with respect of what genetic tests should be performed before their use.

Preimplantation genetic diagnosis (PGD), in most cases an analysis of the one or two biopsied cells of a 3-day-old embryo, was developed in $1989^{4}$ in an effort to avoid the transfer of affected embryos from couples who carried serious genetic disorders, such as haemophilia, cystic fibrosis (CF) or chromosomal abnormalities. Using PGD, unaffected embryos only can be selected for transfer before pregnancy starts and thus the need for a selective abortion after prenatal diagnosis (PND) can be avoided. ${ }^{5}$

There seems to be a general consensus among professionals that the use of PGD is acceptable for medical indications if a high risk of a serious genetic disorder exists. However, PGD has been used not only to diagnose and avoid genetic disorders but also to select for certain characteristics, such as matching tissue type to an existing sibling for therapeutic purpose. In addition, the demands to use PGD for fully nonmedical purposes are increasing (ie, sex selection of embryos solely for social or cultural reasons ${ }^{6}$ ). This extended use of PGD is controversial.

Preimplantation genetic screening (PGS), previously also called 'aneuploidy screening', 'low-risk PGD' or 'PGD-AS', is performed for different indications than PGD by using different methods. PGS is carried out for infertile couples undergoing IVF in order to restrict from transfer those embryos clearly having numerical chromosomal abnormality, with a hope that it will improve poor pregnancy outcomes. Although the use of PGS is steadily increasing, ${ }^{7}$ the importance and place of this technique is still being debated. ${ }^{8,9}$ This discussion is particularly relevant where this genetic method is applied at IVF clinics without the necessary genetic expertise.

PGD and PGS are still rather rare procedures owing to high technical demands, costs, relatively low pregnancy rates and strict licensing procedures in many countries. Very few studies have performed an integrated analysis of technological, patient-related, ethical and economic aspects of PGD and PGS ${ }^{10}$.

In contrast to these hopeful improvements, there are a number of reports on adverse outcomes in children born as the result of ART. Numerically, multiple gestations are clearly the major risk to the future child's health. ${ }^{11}$ There is also a growing concern for structural anomalies and longterm health effects (eg, Bonduelle et al, ${ }^{12}$ Hansen et al ${ }^{13}$ and Klemetti et $a l^{14}$ ). Several studies on the safety of the techniques have been published, but they have been short term or too small to give any certainty. However, large reviews of these studies suggest a slightly elevated risk of birth defects in children born following ART (eg, Hampton, 2004). ${ }^{15}$ Whether this is related to the ART procedure as such, or the 'disease of infertility', is not known. ${ }^{16}$ Many of the new ART techniques, such as biopsy in PGD and PGS and microinjection in intracytoplasmic sperm injection (ICSI) as well as culture media, are feared to be detrimental for the embryo development.

The ART-related legal and ethical issues have been debated at many levels both nationally and internationally. The attitudes towards ART, PGD and PGS vary substantially not only in different parts of the world but also within Europe, owing to scientific, cultural and religious differences. Some techniques are wholly or partly prohibited by law or access to them is limited to married couples or only for specific medical indications. However, as infertility does not respect cultural or religious boundaries, where necessary, couples seek medical fertility treatments outside their own countries. Similarly, couples whose children are at risk of inheriting some severe genetic disease might go to a country where PGD is allowed. Additionally, crossborder transfer of gametes might be needed when treating couples from different ethnic background, and appropriate gametes cannot be found in the treating country. All this demands wider perspective when considering regulation and guidelines.

Several of the existing or possible applications of ART, in particular PGD, are at the interface between reproductive medicine and clinical genetics. As ART was originally used to improve infertility treatments, it is now becoming used for genetic reasons. The development of reproductive sciences and genetics has given a new dimension to ART: as aptly stated in a United Nations Educational, Scientific 
and Cultural Organisation's (UNESCO) report on PGD and Germ-Line Intervention of 2003, 'IVF aims at having a child, PGD aims at having a healthy child and PGD/human leukocyte antigen (HLA) testing aims at having a healthy and helpful child'.

The Public and Professional Policy Committee (PPPC) of the European Society of Human Genetics (ESHG) found it necessary to create professional recommendations on how to use IVF techniques safely and reliably from the genetic point of view. It also held important to issue guidelines on acceptable (genetic) goals of IVF treatment and on how these expensive treatments should be prioritised in the European healthcare systems.

Accordingly, the purpose of the present paper was to outline a framework for development of guidelines for the interface between genetics and ART.

\section{Methodology}

The approach to the topic needed collaboration with other groups involved, especially infertility and reproductive genetics professionals of the European Society of Human Reproduction and Embryology (ESHRE). In addition, collaboration with the Institute for Prospective Technological Studies (IPTS), one of the seven scientific institutes of the European Commission's Joint Research Centre (JRC), was considered as essential, because its mission is to provide European policy makers with technoeconomic analysis to support the policy-making process.

The method used in creating this background document was at first to examine relevant articles and literature and to collect existing laws, practices and professional guidelines mainly from the European countries. The legal framework is presented in Appendix A. These documents were submitted for consultation to approximately 150 high-level experts, such as gynaecologists; geneticists; representatives of patients' organisations for infertile couples and hereditary diseases; as well as to psychologists, ethicists, lawyers, health politicians, social scientists and others.

A group of 50 experts representing the aforementioned different disciplines were later invited to Sevilla on March 31-April 1, 2005 to discuss the issue further. The results of the Sevilla workshops have been adapted to this document.

PPPC drafted the recommendations in collaboration with ESHRE and submitted them for acceptance by ESHG and ESHRE. The aim was to reach recommendations that both ESHG and ESHRE could adapt and convey to the professional societies. The recommendations will be published in European Journal of Human Genetics and in Human Reproduction.

\section{Goals of services}

Possible applications of ART

Fertility treatment aims at achieving a successful pregnancy (= live birth of one healthy child) in the most natural way possible. ${ }^{17}$ Infertility has been described as 'a failure to conceive after at least one year of unprotected coitus'. ${ }^{18}$ The definition of the concepts of infertility, subfertility and sterility has however raised discussion. Subfertility means in general decreased fertility, whereas sterility means total infertility. The European Classification of Infertility Taskforce (ECIT) has been formed by ESHRE to develop a set of infertility-related codes (descriptions, interventions and outcomes) appropriate for computerisation (http://www.ecit.info).

ART is used also when a naturally conceived pregnancy might carry serious risks to the offspring. Factors critical to the success of the treatment are maternal age, embryo quality and number of embryos transferred. The older the woman, the lower is the success rate, the riskier is the pregnancy for both mother and child and the more likely the embryos are to contain sporadic chromosome anomalies.

ART is applied to a variety of indications, most but not all of which are medical. These indications can be divided into the following categories, which, however, are partly overlapping:

Non-genetic or multifactorial medical causes:

- genital, or genital tract anomalies,

- sequelae after previous diseases, traumas or operations,

- toxic agents (chemotherapy, environmental toxins, several pharmaceuticals),

- infections,

- endometriosis, varicocele, etc

- endocrine/hormonal causes,

- sexual dysfunction (vaginismus, anejaculation. etc).

Genetic causes:

- monogenic causes of infertility,

- chromosomal causes (including Y-deletions),

- risk of conveying a genetic disease to a child.

Other reasons:

- premature menopause or postmenopause,

- same-sex couples,

- single females,

- treatment of a sibling (HLA matching),

- HIV discordant couples,

- therapeutic cloning.

Some of the above applications are nonmedical and/or controversial and may not be acceptable to all. However, most of them have already been applied.

\section{IVF clinics and collaboration with genetic clinics}

Reproductive medicine and genetics have long been overseen separately and with very different degrees of care. ${ }^{19}$ While the need for genetic services is increasing at IVF clinics, they are not always appropriately provided.

Family history may indicate a presence of a genetic condition underlying infertility and therefore careful 
family history should be collected routinely. ${ }^{20-22}$ The potential transmission of a genetic disorder to their offspring is a major problem for many at-risk couples when planning a pregnancy. Therefore, some fertile couples seek IVF treatments in order to avail themselves of PGD, for instance, if they regard termination of pregnancy after implantation as ethically unacceptable.

However, the use and interpretation of genetic tests demand expertise and special counselling skills (see below on Counselling), because the results of genetic tests may have serious implications for an individual and his/her family members.

For this reason, ART requires a multidisciplinary teamwork and adequate training of all the professionals involved. Although professionals in genetics have the best qualifications for specific genetic counselling of the couples, also gynaecologists have a duty to give counselling on the possible genetic consequences of ordinary ART techniques, PGS and other relevant issues in case the problem of the couple is infertility without any known genetic indications or consequences.

A European multicentre survey of 20 centres revealed that although many of the activities (IVF, embryo culture and biopsy) take place in IVF units, others (counselling and diagnosis) are at the responsibility of genetic diagnostic centres: in the PGS procedure, the intake of the patients happened predominantly via an IVF unit, whereas for PGD, the patients were often referred via a genetic clinic. According to the survey, the number of monogenic diseases for which PGD was offered varied considerably. In comparison to PND, PGD appeared to be much more

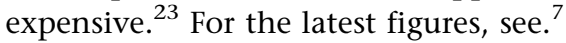

The ESHRE PGD Consortium was established in 1997 to survey the practice of PGD worldwide. Since then, it has published four reports that give an overview on PGD from European centres as well as centres in the US, Asia, Australia and Israel. It will continue this work and in the future focus on centres that take care of the complete PGD cycle. According to the latest report, new indications in PGD and PGS appear with every new report. Noteworthy is the increasing number of PGS cycles performed for male indications and for previous aneuploid pregnancies. The overall pregnancy rate (positive heart beat) per oocyte retrieval was $16 \%$. This relatively low pregnancy rate was anticipated most likely to be a result of the low proportion of embryos suitable for transfer. ${ }^{7}$ Theoretically, it could also be owing to possible embryo damage from the procedure.

Only large PGD centres have enough time and resources to research and develop the tests needed for PGD, ${ }^{24}$ as it is very time-consuming (several weeks or months for each disease) at the single-cell level. Depending on the nature of responsible mutations, many genetic diagnoses that may be available at standard conditions cannot be reproduced at a single-cell level through the available technologies either.

\section{Licenses and surveillance}

In general, all medical professionals work under specific license and overseeing provisions. According to the International Federation of Fertility Societies (IFFS) Surveillance 04, many countries have set up standards for ART clinics and perform surveillance by requiring periodic reports from clinics in order to obtain or maintain a licence. ${ }^{25}$ These procedures are stipulated either by laws or governmental guidelines usually involving a licensing body. In some countries, different clinics have different levels of licences depending on the complexity of the techniques used and the disorders being tested.

\section{Informed consent}

All medical interventions and counselling should be voluntary, and require the informed consent of the patients and donors. Information provision and consent procedures that enable applicants to make informed choices are crucial components of assisted reproduction services. It is important to discern the information component and the consent component of informed consent. ${ }^{26,27}$ It is especially important when genetic tests are recommended, because they may have far-reaching repercussions for the patients, and sometimes for immediate and extended family, and because some uncertainty about the results still exist. ${ }^{28}$

Information provision means that the patient understands the risks, discomforts and benefits of the procedure to be performed and is aware of various alternatives, including the alternative of not performing the procedure. ${ }^{29}$ There is a need to present accurate data of the risks involved, without which the consent may be invalid. Accumulated data increasingly suggest an elevated risk of birth defects after ART (see below). Even where the data are still uncertain, possible risks of ART should thus be addressed when obtaining an informed consent before starting treatment. ${ }^{30-34}$

However, patients seem to interpret the information given within a personal frame of reference and recall it selectively. ${ }^{35}$ Medical professionals are therefore suggested to evaluate patients' understanding, for instance, by asking them to describe the procedure, its purpose, and its risks and discomforts in their own words. ${ }^{29}$

The demand for informed consent and its contents is generally valued and expressly stated in most laws, even though it has been reported of some practical variations in Europe. ${ }^{23}$ Several professional and institutional organisations provide guidance on this issue.

\section{Genetic studies of IVF couples Need for genetic testing}

The reasons behind infertility are manifold, and often remain unsolved. There are no general guidelines to what extent the causes of infertility should be sought and 
practices vary. Sometimes the cause is not found owing to inadequate diagnostic procedures. Testing will, however, often help with the diagnosis, will give information for the treatment and evaluation of the risks to the offspring and, moreover, will enable informed decision-making.

In the absence of general practice guidelines, clinics apply different methods and policies. ESHRE has addressed the issue of 'optimal use of infertility diagnostic test and treatments' in the Capri workshop. ${ }^{20}$ The Italian community of professionals, supported by some international societies, has created guidelines for the appropriate use of genetic tests in infertile couples in 2002. ${ }^{36}$ European Molecular Genetics Quality Network (EMQN) has provided elaborated, disease-specific guidelines (www.emgn.org).

It might be considered advantageous to be able to perform genetic counselling and offer a selection of genetic tests before all IVF treatments, because many genetic causes of infertility still remain unrecognised. In practice, however, providing genetic services in each case is unrealistic, although improvement in this field is needed in any case. As a compromise, it has been recommended to solve reproductive, medical and family history always before initiating the treatments ( $\mathrm{K}$ Aittomäki, 2005, personal communication) and to perform genetic investigations, chromosome and gene analysis in selected cases.

In the case of a family history of a severe (hereditary) disease, but where the couple does not want to use PGD, genetic counselling should be offered before any type of ART, and especially before the use of donor gametes. Patients should be told that despite the testing procedures, absolutely safe germ cells do not exist, whether from a donor or a partner, as only a limited number of hereditary diseases can be detected and other factors also may affect the outcome.

\section{Genetic diagnosis of male infertility}

Approximately 15-20\% of Western males are affected by infertility. Although some reasons can be diagnosed, often no clear cause can be found, and the condition is labelled as 'idiopathic infertility'. The most likely explanation for this is our poor understanding of the basic mechanisms regulating the genetic networks causing human infertility. ${ }^{37}$

In men presenting with defects in sperm, only karyotype and Y-chromosome microdeletion analyses are usually being performed, which will not reveal the majority of genetic abnormalities. ${ }^{38,39}$ Additionally CF transmembrane conductance regulator (CFTR) mutations in cases of congenital bilateral or unilateral absence of vas deferens (CBAVD, CUABV) are often tested. By these analyses, it is possible to identify a genetic cause of severe male infertility in $10-20 \%$ of cases. ${ }^{36,40}$

Karyotype analysis and Y-chromosome microdeletion screening have been recommended to be performed in men with nonobstructive azoospermia, because as many as $10-15 \%$ of these patients have an identifiable abnorm- ality. ${ }^{39,41,42}$ Aittomäki et al $^{22}$ suggest that these tests should also be carried out in men with oligozoospermia below $5 \times 10^{6} / \mathrm{ml}$. Although it has been suggested that karyotype analysis should, before ART, be performed even in normozoospermic men, because some aberrations (such as $47, \mathrm{XYY}$ ) can be found, ${ }^{36}$ this is not a general recommendation. Y-deletions are very rarely found in men with a sperm count above $5 \times 10^{6} / \mathrm{ml}$, but below this count, the deletions are found in $8.2 \%$ of men. ${ }^{43}$ The deletions arise usually de novo, but are transmitted to all male offspring in ICSI. $^{22}$

New findings have shown that not every steroid sulphatase (STS) deletion in Y-chromosome causes male infertility. ${ }^{37}$ The existence of a spermatogenesis-controlling factor(s), called azoospermia factor (AZF), in the long arm of the Y-chromosome was first based on identification of large deletions of this chromosomal area in azoospermic or oligozoospermic men. ${ }^{44}$ Later microdeletions (Yq) were found to be another common cause of male infertility ${ }^{36}$ and might result in azoospermia or severe oligozoospermia. At present, it is known that AZFa, AZFb and AZFc deletions are caused by intrachromosomal recombination events between large homologous repetitive sequences blocks located in Yq11, AZFc being recognised as the most frequently known genetic lesion causing male infertility. ${ }^{45}$ Owing to a possibility of three microdeletions intervals with 'complete' or 'partial' appearance, an estimation of the extension of male's AZF deletion is recommended to be confirmed by investigating testicular pathology, because only complete AZFa and AZFb deletions are associated with a specific testicular pathology, whereas partial AZFc deletions may have no impact on male infertility. ${ }^{37}$

The most common chromosomal aberrations associated especially with severe oligo- and azoospermia are sex chromosome aneuploidies and chromosomal translocations. ${ }^{46}$ Consequently, occurrence of aneuploid embryos will lower the success rate of the IVF treatment, and offspring have a risk of an unbalanced translocation or an aneuploidy. ${ }^{4-49}$ PGS may be useful for some of these couples in making the IVF treatment more successful by choosing the embryos with the best possible developmental potential. ${ }^{22}$ In aneuploidy of the sex chromosomes or Klinefelter syndrome (KS), there are few, if any, spermatozoa in the ejaculate. Couples must either be treated following testicular sperm retrieval (if successful) combined with ICSI or use donor sperm to have children. In general, men with KS produce a higher number of sperm with aneuploidy, particularly of sex chromosomes, with respect to normozoospermic fertile controls and nongenetic idiopathic severely oligozoospermic men. The total amount of aneuploid sperm has been recently quantified as $20 \%$. Men with mosaic KS produce about 5\% of aneuploid sperm. ${ }^{50}$ Testicular sperm can be found in almost one-half of the patients with even nonmosaic KS, and most have normal karyotypes. ${ }^{51}$ Various mosaic karyotypes may cause 
oligozoospermia and can also be treated with IVF using ICSI. $^{5}$

As has been shown by fluorescence in situ hybridisation (FISH) analysis, men with a normal lymphocyte karyotype may still have aneuploidy in their spermatozoa. ${ }^{52-54}$ The frequency of chromosomal abnormalities in spermatozoa of chromosomally normal men is about 10\% (involving 7\% of structural aberrations and $3 \%$ of aneuploidies). The FISH analysis of sperm from infertile men provides essential data on the chromosomal status of sperm before use in IVF and ICSI. When immature sperm retrieved by TESE (testicular sperm extraction) is used, chromosome anomalies in the embryos are more common than when using ejaculated sperm. ${ }^{55}$ World Health Organisation (WHO) recommends that methods need to be developed to detect aneuploid spermatozoa so that they can be excluded from sperm preparations used for ICSI. ${ }^{18}$

Congenital bilateral absence of the vas deferens (CBAVD) is a form of infertility with autosomal recessive genetic background in otherwise healthy males. ${ }^{56}$ The most common cause is CFTR gene mutations. Striking genotype differences are observed in CF and in CBAVD. ${ }^{56}$ The majority of males with $\mathrm{CF}$ have obstructive azoospermia owing to CBAVD. ${ }^{57}$ CBAVD or CUAVD may also occur as the only manifestation of CF. ${ }^{58,59}$ This occurs as a result of a combination of a common or severe CF mutation with a mild CF mutation or with a specific intronic variant. As genetic testing is not able to identify all causative mutations, it follows that a negative test does not exclude the existence of an unknown mutation. However, when a patient tests positive, that is, has CBAVD owing to CFTR mutations, there is a risk of both male and female offspring to have CF and for male offspring to have CBAVD. The risk for the offspring depends on whether or not the spouse is a carrier, since one mutated allele will always be inherited from the affected male. ${ }^{22}$

Even with the current state of knowledge, counselling of the couples with CBAVD remains very difficult. ${ }^{56}$ The genetics of CBAVD is more complex than in $\mathrm{CF}$, as (i) genetic analysis is able to prove but not to exclude the diagnosis of a genital form of $\mathrm{CF}$, and (ii) the risk of CF or CBAVD in the offspring may be unpredictable when rare mutations are identified in the male or the female (see Claustres ${ }^{56}$ for thorough analysis and risk calculations).

The importance of screening for kidney abnormalities in males with CBAVD has been pointed out, as unilateral renal agenesis is sometimes associated with CBAVD (in the absence of CFTR mutations) and renal malformations of varying severity might be transmitted to the offspring. ${ }^{60-62}$

Screening for androgen receptor gene mutations in azoospermic and severely oligozoospermic men is still debatable, given the quite low frequency (about 2\%) of mutations found in these patients. ${ }^{36,40}$

As is known since 1983, males with a normal karyotype may show synaptic anomalies that usually cause chromo- somally abnormal sperm. These are detectable only through the study of meiosis in testicular biopsies. It has been suggested that meiotic studies should be used more often in the study of infertile males. ${ }^{63}$ Synaptic defects are especially frequent in males with a severe oligozoospermia, or with previous IVF failures, even if normozoospermic. In Spain, meiotic studies in testicular biopsies are included in the protocol of study of infertile males. ${ }^{64}$

PGD has been regarded as useful for couples whose infertility is owing to some male factors, such as meiotic anomalies $^{65}$ or CF and CBAVD.

A British article highlights the need for formal training in andrology among those clinicians who are managing infertility in the male, because infertility appears to be treated mainly by gynaecologists who have often had no teaching in the subject of andrology. ${ }^{66}$

The practice of chromosomal testing in connection with ART varies between countries. To mention some practices, in Norway, for instance, chromosomal analysis before ICSI is offered to all couples, in Sweden, only to men with nonobstructive oligo- or azoospermia, and in Finland, the testing is offered to men with nonobstructive oligo- or azoospermia and to their spouses. ${ }^{22}$ In case of agenesis of the vas deferens, mutations in the CFTR gene are generally studied, in both spouses when needed, to avoid transmitting CF to the offspring. ${ }^{67}$ Some centres in Italy offer sperm aneuploidy evaluation with FISH in patients with repeated implantation failure and recurrent abortion, but this application is still held as experimental. ${ }^{68}$

To summarise, special consideration is needed when treating infertile men, since infertility may be caused by abnormalities that may cause infertility and/or potential serious diseases to the offspring. ${ }^{36,40,69}$ Counselling and more thorough genetic testing should be carried out before any ART treatments, to inform patients of the risks and to find appropriate treatment in each case.

\section{Genetic causes of female infertility}

Also, a number of causes behind female infertility may lie in chromosomal aberrations and gene mutations. The possibility of single or multiple gene defects in common clinical conditions, such as polycystic ovarian syndrome, or premature ovarian failure, has been described. In case of female infertility, several tests during the diagnostic workup can be relevant before ART, such as karyotype analysis, CFTR gene and fragile-X. ${ }^{36}$ In addition, other genetic causes like mutations in LH and FSH receptor genes as well as structural abnormalities of the female genital organs may cause female infertility. Repeated early miscarriages owing to a balanced translocation, either in the female or the male, may be connected to infertility. Often, the treatment is based on donated oocytes or embryos and the genetic risks thus need not to be considered. However, in case of a congenital malformation with a very abnormal or missing uterus, the only treatment might be ART with the 
female's own oocytes and a surrogate mother. In such a situation, there could be possible genetic risk of inheriting female infertility. Nevertheless, no case has been shown among the children born, though.

Indications set by American Society for Reproductive Medicine (ASRM) for considering the use of donor oocytes include women with hypergonadotropic hypogonadism (premature ovarian failure); women with advanced maternal age; women who have a diminished ovarian reserve; women who are known to be affected by or are carrier of significant defects or have a particular family history; women with poor oocyte; and/or embryo quality or multiple failures during prior attempts to conceive via ART. ${ }^{70}$

Tests to be carried out for couples who receive donated gametes or embryos

ASRM's guidelines on evaluation of oocyte recipients include obtaining medical and reproductive history, physical examination, laboratory tests, psychological evaluation and assessment of the uterine cavity. Also the partner should undergo several tests and an evaluation process. ${ }^{70}$ General guidelines would also be needed in Europe, even though some clinics follow the ASRM's guidelines and a few countries have their own. ${ }^{71}$ For instance, testing the male partner (or sperm donor) for carrier status of some relatively common autosomal recessive diseases in the population concerned could be required to minimise the risk of such diseases in the offspring.

\section{Is success rate an issue?}

It has been claimed that the practice of publishing success rates of identified clinics has impacted on the range and availability of treatments. ${ }^{3}$ It may also have undesirable impact on the methods used, and, for instance, result in multiple pregnancies. Presenting success rates to the public requires responsibility and generally accepted standards to avoid unjustified expectations and comparisons between centres.

Some indications of infertility include an extremely low success rate of pregnancy. Therefore, the patients may be selected on the basis of potential success rate. Moreover, embryo biopsy during PGD has been suspected to decrease success rates of ART, whereas in some situations, it may increase possibilities for a healthy life in the future child.

At the moment, success rates are presented in many ways, for example, for some, a positive heart beat of the embryo means success, whereas others may consider success only the birth of a healthy child. Success rates reflect different components of the treatment, such as expressions 'per transfer', 'per oocyte pick-up' or 'per cycle started'. Other variables include that the probability of success is higher in the first new cycles; younger women are more fertile; multiple pregnancies increase reported success rates; cancellation rates have a negative impact on success rates; and the number of embryos transferred will also impact on the reported result. ${ }^{3}$ Lately, many Scandinavian centres perform single-embryo transfer and score success rates in terms of clinical pregnancy rates per oocyte pick-up (including both fresh and frozen embryo transfers $^{72}$ ). There is evidence that a transfer of more than one embryo at a time does not improve the likelihood of pregnancy, but only increases the likelihood of multiple pregnancies. ${ }^{73}$ The provision of 'delivery of a single, term gestation, live baby per cycle initiated' has been introduced as a new standard of success for IVF clinics. ${ }^{17}$ Also, preterm singleton live births are proposed to be noted then. ${ }^{74}$

\section{PGD and screening PGD vs PND}

PND is a diagnostic or presymptomatic test carried out on a developing fetus through amniocentesis, chorionic villus sampling (CVS), foetal blood sampling, collecting foetal material in maternal circulation or ultrasound. PND is used to detect a fetus with a chromosomal aberration, congenital malformation or disease, or that is at risk for a disease and thus offers the parents the option to terminate the pregnancy in order to prevent the birth of offspring with genetic and/or congenital anomalies. Couples who have not experienced prenatal testing before do not generally opt for PGD, but first try to conceive spontaneously, and ask for PND. Those who have experienced one or more terminations of pregnancy following PND are more prone to choose PGD. ${ }^{75}$

PGD was introduced at the beginning of the 1990s as an alternative to PND to avoid termination of pregnancy for couples with a high risk of their offspring being affected by a sex-linked genetic disease. At that time, embryos obtained in vitro were tested using molecular techniques to ascertain the absence of a Y-bearing sequence, and only female embryos would be transferred. Since then, techniques for molecular and cytogenetic analysis at the singlecell level, including assessment of first and second polar bodies from oocytes or blastomeres from cleavage-stage embryos, have evolved considerably. ${ }^{24}$ The list of diseases for which PGD has been used is slowly growing.

\section{Techniques for genetic analysis}

The two main techniques of obtaining nuclear material for genetic analysis are the aspiration of one or two polar bodies from oocytes or the removal of one or two blastomeres from early embryos (cleavage stage biopsy). ${ }^{76,77}$ The most commonly used biopsy method is cleavage stage biopsy. ${ }^{7}$ A third, less used method, is trophectoderm biopsy from blastocysts. ${ }^{78}$

Polar body biopsy The stem cells (oogonia) of the future oocytes enter the process of first meiosis during early oogenesis. The chromosomes are duplicated ('replication') and chromosomal material from the paternally and 
maternally derived chromosomes is exchanged (the process of recombination or crossing-over). The primary oocyte is at this stage diploid with duplicated chromosomes, and remains fixed in prophase until triggered by the preovulatory LH surge to complete the first meiotic division. The first polar body is extruded as a by-product of that division, which normally occurs inside the follicle, before the oocyte is ovulated. The first polar body thus contains the counterparts of the chromosomes of the developing oocyte. As the oocyte is surrounded by the zona pellucida ( $\mathrm{ZP})$, the polar body remains 'trapped' in the space between the oocyte and the ZP. Following fertilisation and activation by the sperm, the oocyte enters the second meiosis, when the duplicated chromatids of the now haploid oocyte separate: one set of chromatids remains in the oocyte, whereas the other is expelled with the second polar body. Owing to the recombination of chromosomal material during meiosis, the polar bodies and the oocyte contain unique genetic material, but should contain the reciprocal chromosomal constitution. ${ }^{79}$ For PGS for aneuploidy, both polar bodies can be removed simultaneously from pronuclear zygotes, whereas in PGD for single-gene disorders, they should be removed sequentially. ${ }^{80,81}$

Polar body biopsy maintains embryo integrity as only byproducts of meiosis are used for analysis. ${ }^{82}$ The polar bodies can be analysed at the chromosome and at the monogenic level. The advantage is that the selection process can be carried out at a very early stage of fertilisation (eg, early pronuclear stage) when syngamy has not yet occurred. Polar body biopsy is used in Germany, for instance, where embryo biopsy for PGD on cleavage stage embryos is forbidden. ${ }^{83}$

Polar body biopsy is a safe technique, if the drawbacks are kept in mind and the risks are correctly estimated. ${ }^{81}$ The main drawback is that polar body analysis can only detect maternally transmitted genetic or chromosomal abnormalities, whereas paternally derived defects and those originating after fertilisation during the first embryonic divisions (postzygotic) cannot be diagnosed. ${ }^{82}$ Another significant disadvantage of polar body diagnosis, as compared to blastomere biopsy, is that in the case of Xlinked or autosomal recessive disorders, the discarded oocytes include those that could have developed into a child unaffected by the disease, depending on the genetic constitution of the sperm. Furthermore, the same oocyte/ embryo is manipulated three times, once for removal of the first polar body, second time for ICSI and then for removal of the second PB. This is very demanding. An important limitation is also the low quality of polar body chromosomal spreading, which can significantly limit the accuracy and the reliability of FISH analysis on this material.

In a large series of first and second polar body analyses for single-gene disorders, a genetic disorder was correctly detected in $98 \%$ of oocytes tested. ${ }^{84}$
Cleavage stage biopsy Cleavage stage biopsy, also called blastomere biopsy, is the most commonly used biopsy technique. Embryos are grown in vitro until they reach their third division (eight-cell stage), which normally occurs on the third day after insemination. ${ }^{24}$ At this stage, the embryos are biopsied to obtain one or two individual blastomeres for analysis. During analysis, the embryos are kept in culture and continue dividing until transfer of the unaffected embryo is performed. ${ }^{5}$

All the cells in a human embryo, at four- or eight-cell stage, are believed by many to be totipotent, that is, none of the cells is yet committed to a specific developmental path. However, it is important to realise that all blastomeres at the four- to12-cell stage are not identical, but express different regulatory proteins. ${ }^{85}$ The developmental consequences of PGD in respect of functional polarisation of blastomeres need more evaluation.

There is no consensus on the number of blastomeres that can be removed safely during cleavage stage embryo biopsy. The decision whether to remove one or two cells is based on many factors including the embryo cell number and the accuracy and reliability of the diagnostic test used. If removal of two cells is contemplated, it is recommended that only embryos with six or more cells are used. ${ }^{77}$

PGD at the cleavage stage has the advantage of testing disorders of both maternal and paternal origin, and those originating after fertilisation. Data from the clinical outcome of biopsied embryos have demonstrated that approximately one-quarter of the cycles end in a pregnancy, but because more than one embryo are transferred per cycle, the implantation rate is lower. ${ }^{7,82}$

There is substantial evidence for significant chromosomal mosaicism in cleavage-stage embryos. Therefore, the biopsied cells may not be representative of the whole embryo. ${ }^{9,86}$

Blastocyst biopsy The blastocyst stage is the latest stage at which an embryo can be biopsied. At this stage, 5-6 days after fertilisation in the human, the embryos contain approximately 150 cells, consisting of inner mass cells and trophectoderm cells. Removal of trophectoderm cells during blastocyst biopsy is achieved by herniation through the ZP followed by laser or mechanical excision. The advantage is that more cells can be obtained; the disadvantages being that usually less than $50 \%$ of embryos reach that stage in culture, and that there is little time left for the diagnosis, as embryos should be transferred before day 5 or $6 .^{24}$ The clinical application of this technique is recent and only limited data has been reported. An Australian study reports on the advantages of testing five or six cells, leaving the inner cell mass intact and enabling the embryos to be electively transferred one at a time, without diminishing the chance of pregnancy compared with cleavage-stage biopsy and testing. ${ }^{78}$ However, one US study suggests that compared to day 3 embryo transfer, 
blastocyst transfer appears significantly to increase the incidence of gestations with monozygotic twinning. ${ }^{87}$ The applicability of blastocyst biopsy on a large scale needs validation. $^{80}$

Analysing methods Two methods have thus far been used for genetic analysis: FISH, the most frequently used for the analysis of chromosomes, and the polymerase chain reaction (PCR) for the analysis of genes in cases of monogenic diseases. ${ }^{24}$ The primary use of FISH was to determine the sex chromosome content of the embryos from couples at risk of various sex-linked disorders, such as haemophilia, but has since been extended to PGD for chromosomal abnormalities and PGS. Amplification of a specific region of DNA by PCR allows for the analysis of disease-causing changes in DNA or markers linked to the disease (eg, microsatellites, SNPs). ${ }^{5}$ Comparative genomic hybridisation (CGH) combined with PGD is a relatively new and still experimental technique. The advantage of $\mathrm{CGH}$ is that the whole chromosome complement is analysed, although polyploidy and balanced translocations cannot be detected. The disadvantage is that the whole procedure takes about $72 \mathrm{~h}$, which limits its use. ${ }^{24}$ Technical improvements are on their way to decrease the time necessary to reach a diagnosis and to improve the resolution through the use of microarrays.

The main indications for PGD

Monogenic disorders and chromosomal rearrangements Couples have an increased risk of conceiving a child with a genetic disease, if they are carriers of a monogenic disease or of chromosomal aberrations. ${ }^{24}$ These diseases fall into the following main categories:

- autosomal recessive (eg, spinal muscular atrophy, TaySachs, CF) with a 1:4 risk;

- $X$-linked recessive (eg, haemophilia A and $\mathrm{B}$, Duchenne muscular dystrophy) with a 1:2 risk in males;

- autosomal dominant (early onset, for example, myotonic dystrophy, achondroplasia or late onset, for example, Huntington's disease (HD)) with a 1:2 risk;

- chromosome rearrangements (eg, reciprocal or Robertsonian translocations; inversions, etc) risk varying with the type;

- mitochondrial, risk uncertain.

The most common indications for PGD for autosomal recessive diseases have been $\mathrm{CF}, \beta$-thalassamia and spinal muscular atrophy. For autosomal dominant disorders, the most common indications are myotonic dystrophy, HD, amyloid polyneuropathy and Charcot-Marie-Tooth disease. PGD for X-linked diseases has been performed for fragile-X, Duchenne muscular dystrophy and haemophilia A. ${ }^{7}$

The number of PGD for reciprocal and Robertsonian translocations has been on the rise, especially because many of the patients who carry such a translocation are infertile or experience repeated spontaneous miscarriages. Robertsonian translocations carry reproductive risks that are dependent on the chromosomes involved and the sex of the carrier. ${ }^{88}$ PGD is especially valuable in those cases where the translocation causes infertility, because PGD is the only way that patients can avoid repetitive abortions. For fertile couples, careful risk assessment and genetic counselling should precede consideration for PGD.

Concerning PGD for reciprocal and Robertsonian translocations when the carrier is a male partner, a preexamination could consist of the chromosomal sperm analysis and the subsequent determination of the meiotic segregation pattern of translocated chromosomes. This assay gives an indication of both the frequency and the types of imbalances in the sperm of the patient, and thus could help with a decision about the relevance of PGD (F Pellestor, personal communication). However, it should be kept in mind that there is not a perfect correlation between the frequencies in sperm and after fertilisation.

PGD for an autosomal dominant late-onset disease with full penetrance of the mutation and the example of HD Some critics argue that testing for late-onset disease is unjustified, because the child will probably have several decades of unimpaired living and the disease may become treatable. This critique, however, is debatable in case of $\mathrm{HD}$, for example, since it is a serious, even lethal, disorder and has complete penetrance of the underlying mutation. A person found to have a mutation would inevitably get the symptoms in the future. Symptoms may appear from the late 20s, but more usually in the fourth or fifth decade. Furthermore, the prospect of the fate of the children who carry HD often imposes an extremely severe burden. ${ }^{27,89-91}$ $\mathrm{HD}$ is currently untreatable. A predictive test allows asymptomatic at-risk adults to know whether they have the Huntington mutation. The use of PGD for asymptomatic individuals with the Huntington mutation and excluding embryos with the mutation is generally regarded as acceptable. ${ }^{76}$ New molecular tools have been developed improving the diagnosis for HD using PGD. ${ }^{92,93}$

Theoretically, nondisclosure PGD could be a solution for some families when the prospective parent at risk for the late-onset disease does not want a predictive test to be informed about his or her own carrier status, but wants to have a child without the mutant gene, for example, in HD. Embryos could directly be tested for the presence of the mutation without revealing any of the details of the cycle or diagnosis to the prospective parents. ${ }^{94}$ This method is controversial and not generally approved by professionals. ${ }^{26,92,95}$ Nondisclosure tests put the practitioners in an ethically difficult position, for example, having to undertake PGD cycles even when the results of previous cycles preclude the patient being a carrier, or having to do mock transfers if no embryos are available. Therefore, the ESHRE ethics task force ${ }^{76}$ discourages nondisclosure 
testing. For at-risk persons who do not want to know their own status, they encourage preimplantation exclusion testing using linked DNA markers to determine whether the HD allele of the embryo from the at-risk parent is originated from the affected or nonaffected grandparent. The mutation itself is not analysed. ${ }^{92,93}$ However, exclusion PGD is considered as ethically dubious by some because embryos with the allele from the affected grandparent will be excluded for transfer, although only in half of the cases the allele will be affected. If the linkage analysis indicates inheritance from the grandparent with $\mathrm{HD}$, the embryo has the same $50 \%$ risk of being affected with HD as the intervening parent. ${ }^{91}$ Notwithstanding their respect for the prospective parents' autonomy, some professionals have problems with the use of exclusion preimplantation testing as well as nondisclosure PGD. The reason is that in both situations an invasive IVF and PGD procedure - with some associated risks - is used while at least half of the couples have no risk at all for transmitting the mutation and could know this with certainty should they use a predictive test. (The same consideration applies to prenatal exclusion testing for HD.)

Offering PGD for patients showing symptoms of HD is presently considered controversial, although $\mathrm{HD}$ is a regular indication for $\mathrm{PND}$, which may result in terminating the pregnancy. For the time being, the decision as to whether or not to allow PGD for a symptomatic parent should be settled on a case-by-case basis with respect for on the one hand, the future child's circumstances, if a parent is to die during childhood, and on the other hand, the parents' autonomy and reproductive freedom. ${ }^{91}$

An extension of PGD for the detection of susceptibility genes for common late-onset diseases and their hereditary subgroups Contrary to the monogenic late-onset diseases discussed in in the previous section, in which the mutated gene inevitable leads to the disease, susceptibility genes increase the risk of developing the disease, but their effect is also being modified by other genes and other factors. It is a dilemma whether it is ethically acceptable to test and select embryos because of an increased risk instead of a certainty - of developing a particular disease later in life. One problem with susceptibility testing relates to the type of test result that could be generated and the validity of the risk figures, as much uncertainty remains. ${ }^{96}$ For the hereditary forms of breast cancer (owing to BRCA1 and BRCA2) and for some hereditary forms of colorectal cancer (eg, familial adenomatous polyposis (FAP) and HNPCC), the absolute risk figures are relatively high, but there are preventive measures. Controversy surrounds the use of PGD in this situation. Polymorphisms increasing the relative risk for common multifactorial diseases are so common that it is hard to imagine that they could be an indication for PGD, because it would mean that an 'indication' for such a test would exist in most families.

\section{Indications for PGS for aneuploidy}

PGS (for aneuploidy) is a method used at present to identify the most chromosomally normal embryo for transfer in an IVF/ICSI cycle. Usually five to nine pairs of chromosomes are examined.

The use of PGS has increased tremendously during recent years in IVF cases without any previously known familial risk of affected offspring, with the aim to improve IVF results (i) in women of advanced maternal age, or (ii) in whom the embryos have repeatedly failed to implant, and (iii) in women who have recurrent miscarriages (not owing to constitutional chromosomal aberrations).

Aneuploidy resulting from nondisjunction increases with maternal age, whereas polyploidy and mosaicism appear irrespective maternal age, and are associated with poor embryo morphology. ${ }^{24,86}$ Maternal age as such is also suggested to increase the incidence of mosaicism. ${ }^{97}$ Various studies on spontaneous abortions have shown that more than half are associated with chromosomal abnormalities. Using a set of specific fluorescent-labelled DNA probes, the most common chromosomal anomalies found are trisomies: trisomy 21 (Down's syndrome), trisomies 13, 16, 18 and 22; and numerical abnormalities of the sex chromosomes $\mathrm{X}$ and Y. ${ }^{98}$ PGS can be enhanced by new technologies, such as $\mathrm{CGH}$, to enable full karyotyping of single cells. $^{99}$

Strategies to improve prognosis of IVF/ICSI include, inter alia, microscopic evaluation of the morphological quality, the dynamics of the cleavage pattern up to the blastocyst stage of the in vitro-cultured human embryo in vitro and, lately, PGS to exclude aneuploid embryos. ${ }^{9}$

PGS may lead to confusion between embryo viability screening and screening for chromosomal disorders during pregnancy. Some chromosomal aberrations (tris 21, XYY, etc) can also result in viable embryos. There is as yet no agreement about the indications for such screening and the legal status of the method varies between countries. ${ }^{100,101}$ Among the member centres of the ESHRE PGD Consortium who contributed data for the last data report, ${ }^{7} 21$ out of 38 performed PGS (K Sermon, personal communication)

Experience of the efficacy, reliability and safety of PGS is growing, but still limited. ${ }^{7,8,102}$ Therefore, some consider this procedure still as experimental. ${ }^{25}$ PGS raises concern, because of the risk of misdiagnosis in part owing to the high rate of mosaicism in cleavage-stage embryos ${ }^{9}$ and technical failures inherent in the FISH technique. Others suggest that PGS would be particularly beneficial for poor prognosis IVF patients. ${ }^{103}$ At present, the advantages of applying this technique on a larger scale have not been demonstrated and harder data are asked for. ${ }^{76}$ UNESCO/International Bioethics Commission (IBC) regards aneuploidy screening to be as ethically acceptable ${ }^{98}$ and also ESHRE and Preimplantation Genetic Diagnosis International Society (PGDIS) have included PGS in their guidelines. ${ }^{77,80}$ 
The first large-scale prospective randomised controlled clinical trial investigating in vitro blastocyst culture and PGS for the selection of the embryos in couples of advanced maternal age, that is, 37 years of age or more, was performed during 2000-2003. As a conclusion, the trial provided no arguments in favour of PGS for improving clinical outcome per initiated cycle in patients with advanced maternal age as such. On the other hand, patients having a transfer after PGS may have an advantage in countries with strict regulation on the number of embryos to transfer. ${ }^{9}$

\section{Differences between PGD and PGS}

PGD was designed for an a priori fertile couple that has a high genetic risk of having an affected child, whereas PGS is provided for an infertile couple to detect certain anomalies of the embryo, which might prevent a successful pregnancy. Indications are hence totally different.

High genetic risk patients are usually selected through genetic centres and might then undergo IVF treatment and PGD, whereas PGS patients become subject to PGS via IVF clinics. ${ }^{77}$

In PGD, the genetic defect is known and established in the parent(s) who carries the defect, whereas PGS is screening for aneuploidies, in case of a possibility of an increased but unspecified risk.

\section{PGD-HLA typing in families where an HLA-matched sibling is desired}

There are a few malignant and nonmalignant (usually genetic) diseases, which are treated by means of bone marrow transplantation. ${ }^{104}$ Allogeneic haematopoietic stem cell transplantation (HSCT) is the only presently known cure for most of these diseases. The method requires the availability of a HLA matched donor who may be an HLA-identical sibling or, alternatively, a volunteer-unrelated donor. The chance that a particular sibling is HLA identical is theoretically $25 \%$. Where there is failure to find a compatible relative as a donor, a search of the worldwide registers for HLA compatible donors can be made. A third possibility is that of obtaining compatible stem cells from cord blood. These are collected at birth and cryopreserved in cord blood banks on a voluntary or commercial basis. The overall success rate of a HSCT in a child with a sibling donor is substantially higher than performed with alternative donors. ${ }^{105}$

PGD with HLA tissue typing is an additional step to determine the tissue compatibility of unaffected embryos with an existing sibling. There are cases of the use of PGD for this purpose, and after birth, the new child's cord blood has been collected and stored, and subsequently used to replace the marrow in the affected sibling, thus curing the disease. Opinion is divided on this issue of having a 'saviour sibling'. A number of groups have addressed the issue, ${ }^{106-108}$ and the practice is on the rise (H Van de Velde, personal communication). Consequently, it has been suggested that application of PGD in combination with HLA typing is a promising therapeutic tool for an affected sibling. 109

Before the existence of PGD, natural conception followed by PND, and possibly termination of pregnancy, was the only alternative, when trying to find a HLA-matching future sibling. ${ }^{105,110}$

\section{No unaffected or diagnosed embryos available for transfer}

It often happens that no unaffected embryos are available for transfer. In such cases, transfer of affected embryos is not recommended. ${ }^{77}$ It may also happen that a diagnosis of some embryos cannot be reached. If PGD was performed to detect monogenic diseases, transfer of undiagnosed embryos is not recommended. In contrast, transfer may be regarded acceptable after PGS and after PGD for certain chromosome rearrangements where they would give rise to nonviable pregnancies. ${ }^{77}$ However, transfer is not generally recommended, unless no other options exist and the couple is informed. ${ }^{80}$ Professionals performing PGD told in Sevilla that none of them transfer embryos that are not diagnosed.

A difficult situation occurs in particular, if none of the embryos, healthy per se, tested mainly for HLA matching has the right tissue type. Similarly, potential selection against healthy embryos carrying some autosomal recessive disorder, such as $\mathrm{CF}$, to avoid transgenerational risk, is not considered ethically justified by many. These issues require further discussion.

\section{Results and misdiagnosis}

It has been estimated than more than 6000 clinical cycles of PGD/PGS have been performed worldwide, with a current estimate of 1000 cycles annually. ${ }^{111}$ In particular, the use of PGS is on the rise ${ }^{7}$ and its possible but nonproven potential to improve IVF results is gaining more approval. ${ }^{9,103}$

The PGD pregnancy rates tend to be somewhat lower than for IVF in general. ${ }^{7}$ Results from 10 years data collection in the Netherlands did not report misdiagnoses and none of the babies had congenital abnormalities, and as a conclusion, PGD was suggested to be a reliable and successful method, with pregnancy rates similar to those of IVF or ICSI. ${ }^{112}$

A systematic New Zealand review of the quantifiable harms and benefits of PGD observed that the incidence and nature of obstetric and neonatal complications after PGD were comparable to those reported after IVF alone, and related mainly to the risks associated with multiple gestations. ${ }^{113}$ The incidence of major birth abnormalities was about $3.8 \%$, which again is similar to that reported after IVF alone. The review concluded that PGD is a 
promising approach, but it is important not to overstate its potential.

According to the fourth ESHRE PGD consortium data collection published in $2005,^{7}$ the clinical reports showed three misdiagnoses out of 136 foetal sacs tested, making the total misdiagnosis rate $2.2 \%$. One misdiagnosis occurred in PGS after FISH $(45, \mathrm{X}$ seen on PND) and two after PCR for monogenic diseases (amyloid polyneuropathy, born and $\mathrm{CF}$, born). Consequently, the misdiagnosis rate after FISH (1:114) was $0.9 \%$, whereas the rate after PCR (2:22) was $9.1 \%$. In previous years, the total rate has been 2-3\% (ESHRE data III, 2002).

Most PGD centres worldwide recommend the use of amniocentesis or CVS in women who become pregnant after PGD in order to safeguard against diagnostic error or a serious unscreened-for foetal abnormality. There is good evidence that these tests are reliable, although they increase the miscarriage rate by $0.5-1 \%$ above the baseline risk, which is estimated at $12 \%$ for the general population.

Mosaicism is said to be a common characteristic in human embryos generated in vitro, which may lead to misdiagnosis. ${ }^{9,82}$ The consequences of chromosomal mosaicism for human embryonic development are unknown. Therefore, detecting and discarding mosaic embryos may imply an important loss of potentially viable normal embryos. $^{9}$

For potential adverse effects, see below.

\section{Patient's attitudes}

A study performed in the UK and Spain on the experiences of PND and PGD by couples who have been exposed to both forms of diagnostics suggested that the experience of PND and subsequent termination of pregnancy can be an unwelcome memory and hence needs an alternative approach. ${ }^{114}$ According to the study, PGD was acceptable to most patients and offered a valuable alternative to PND, but undergoing IVF itself was associated with stress and anxiety: $41 \%$ of patients found the PGD cycle extremely stressful, especially the time waiting after embryo transfer for a pregnancy result and time waiting after the initial consultation before a treatment cycle. The patients regarded as the main advantage of PGD that only unaffected embryos were transferred to the uterus and thus therapeutic termination could be avoided. The low success rate was considered the main disadvantage. More than $90 \%$ of couples used and were satisfied with genetic counselling. In all, 77\% would choose PGD again in a further pregnancy attempt. An Australian study ${ }^{115}$ also concluded that the patients found PGD to be a highly acceptable treatment and morally less problematic than abortion. The recommendation of performing back-up PND once the pregnancy has started and the possibility of an abortion in the event of a misdiagnosis raised most concern in nearly half of the patients. The issues surrounding the transfer of embryos, restrictions to PGD and the destruction of embryos raised some different thoughts. ${ }^{115}$

Factors predicting couples to start with PGD are the number of previous spontaneous or induced abortions, and the absence of acceptable alternatives and openness about the treatment. ${ }^{75}$ Couples tend to feel it as a duty to first apply PGD to avoid termination of pregnancy and they want to avoid recurrent abortions. Couples usually underestimate the burden of PGD beforehand. ${ }^{75}$

\section{Sex selection for medical reasons}

Many countries and organisations (UNESCO, COE and ASRM) accept sex selection for genetic reasons to prevent the birth of a child with a serious condition. Usually this occurs in situations wherein the specific mutation at the origin of an X-linked recessive disease cannot be detected.

A more controversial indication for sex selection is the prevention of the birth of a daughter who is carrier of the mutant X-linked recessive gene. Indeed, when the future father has a recessive X-linked condition, all his daughters will be carriers, but sons healthy. Thus, the couple might want to have only male offspring to avoid the birth of a carrier daughter and the problems that it brings along. It is clear that in this situation sex selection is used to prevent the implantation of a carrier embryo.

The situation described above has a lot in common with the situation whereby direct mutation analysis is possible, and whereby neither female carrier embryos nor affected male embryos are implanted (because the parents only want unaffected boys or daughters who do not carry the mutation).

The evaluation of the situation is even more complex for some X-linked diseases, because female carriers of the mutant X-linked gene may also have an increased risk of symptoms, although less severe than males carrying the same mutation.

Sex selection for social or family-balancing reasons Sex selection for other than health purposes has lead to vivid debate recently. ${ }^{116-120}$ The mere fact that ESHRE included sex selection in its report in the first place has caused disapproval. ${ }^{121}$ However, the attitudes towards preconception sex selection for social or family-balancing reasons vary among cultures, which is noteworthy in the pluralistic Europe. Still, the European Convention on Human Rights and Biomedicine, ${ }^{122}$ Article 14, explicitly bans sex selection for other than health purposes by stating that, 'The use of techniques of medically assisted procreation shall not be allowed for the purpose of choosing a future child's sex, except where serious hereditary sexrelated disease is to be avoided.' However, some ethicists question the general ban and fail to see the moral wrongness of social sexing or family balancing (eg, $\mathrm{Dahl}^{120}$ ), some referring also to the concept of 'reproductive liberty', the ignorance of which requires justifica- 
tion. ${ }^{117,118}$ Some argue that if family balancing were allowed, fears of sexism would lack ground, because both sexes were presented in the family. ${ }^{123}$ However, public opinion has been reported to be against this. ${ }^{123,124}$

\section{Regulation}

PGD is totally banned in some countries, whereas most are content to limit the indications for PGD to serious disorders or conditions. This includes the detection of the sex of the embryo in order to avoid serious hereditary Xlinked disease. According to a recent comparative study, there is symmetry in both the substantive requirements (ie, severity, only health indications) and procedural safeguards (ie, informed consent, counselling, confidentiality, oversight and accreditation) surrounding reproductive genetic testing. The degree of severity or probability of the risk has not been defined further, though. Under the survey, common feature is that the final decision is medical. ${ }^{6}$

Germany offers an example of a strict regulation, as there the Embryo Law is interpreted as to prohibit other PGD than polar body diagnosis, However, this interpretation has been debated. ${ }^{79,125}$ According to a recent survey, public attitude is more liberal towards PGD than public policy. ${ }^{125}$ At present, German patients travel to Belgium or to Spain, for instance, to get the treatment they desire. ${ }^{126}$

Some have argued in favour of tight regulation of PGD, because they think that otherwise this new technique might be brought into disrepute owing to its application, for instance, to sex selection. ${ }^{100}$ These demands have been supported by concern that PGD may be seen as eugenic, either through its aim to reduce the number of people with a genetic disorder or because it makes possible selection of embryos on the basis of nonpathological characteristics, leading to full-blown free-market eugenics. ${ }^{127}$ However, ethicists often answer to these doubts by a question 'what's wrong with eugenics' (see later section 'What is wrong with eugenics?'). The fact that someone may use a specific technique elsewhere for purposes that are illegal, unethical or unsafe is not necessarily indicative of this being a slippery slope to perdition down which we all are inevitably doomed to slide.

The Human Genetics Commission (HGC) in the UK, for instance, has given the following recommendations to the HFEA: PGD should be limited to the detection of specific and serious conditions; PGD should not be used for trait selection or in such a way that it could give rise to eugenic outcomes; consistency is needed between conditions considered as appropriate for PGD and for PND. PGD to detect carrier status for an autosomal recessive condition should, where possible, be avoided. Guidance regarding PGD to select and implant embryos that are affected by a genetic condition has not yet been formulated. ${ }^{124}$

Guidelines and recommendations of the ASRM, ESHRE, HGC, HFEA, PGDIS, UNESCO/IBC and WHO are now amending the lack of generally accepted rules concerning PGD. ESHRE and PGDIS, for instance, have published their best practice guidelines in 2004 .

\section{Selection of donors based on genetic information What type of tests are carried out?}

When donors are chosen for oocyte or sperm donation, many clinics have the practice of asking family history, usually focusing on first-degree relatives, and routinely performing some medical tests (ie, hepatitis, HIV and venereal diseases) and genetic tests at least in cases where family history indicates a risk of hereditary disease.

Upon WHO's recommendation sperm donors should be screened for hereditary as well as infectious diseases. ${ }^{18}$ This was, however, not fully accepted by the workshop participants in Sevilla, who mostly viewed that the genetic testing of donors should not lead to selection of better genes for donor-conceived children compared to genes presenting among the general population.

There are no common European guidelines on testing the donors. In the United States, the ASRM has issued guidelines on gamete and embryo donation, which include exhaustive lists of risks and tests as well as minimum genetic screening criteria for gamete donors (also specified by ethnic groups ${ }^{70}$ ). Some European centres (eg, one centre in Belgium) follow ASRM's guidelines for gamete and embryo donation.

Although acknowledging that all reasonable tests should be performed to prevent transmission of a genetic disorder, the HFEA, for instance, notes that genetic testing should be limited to the determination of a carrier status for inherited recessive disorders in which abnormal test results carry no significant direct health implications for a prospective donor, either sperm or oocyte donors. Certain tests (CF, Tay-Sachs, thalassaemia and sickle cell disease) are recommended depending on the population the donor belongs to. ${ }^{71}$ The HFEA states that centres are expected to ensure that where prospective donors are genetically tested, they have the same level of support and counselling as recipients. $^{128}$

A large international commercial distributor of gametes (Cryos in Denmark) checks the karyotype (46, XY). Upon consideration case by case and following its Medical Standard Operation Procedure, Cryos will examine genetic diseases of the donor candidate (sickle cell disease, thalassemia, Tay-Sachs disease, CF, etc, the list is increasing). The donor's complete medical history (specifically regarding possible heritable conditions) is recorded, and the data are entered in a registry of donors. In Cryos's opinion, recipients generally want as extensive genetic disease screening as possible, whereas donors, on the contrary, are usually against genetic disease screening, as it would be traumatic for them to receive positive results and consequent rejection. Donor rejection should only be 
enforced when known risk of a genetic disease is greater than that in the general population (www.cryos.dk).

According to the 'IFFS Surveillance 04' of practices in different countries, genetic screening by history in sperm or oocyte donors is widely practiced. ${ }^{25}$

In the European Union, several tests will be obligatory in the future when procuring reproductive cells, if technical requirements of the Tissue Directive come into force as proposed (see Appendix A). The use of reproductive cells shall meet certain criteria. Donor selection criteria and laboratory testing is not needed in the case of partner donation for direct use, that is, when cells are donated within a couple and used without banking. The proposed laboratory tests shall basically include HIV, hepatitis B and C. Donors in connection with high incidence areas shall be tested for HTLV-I antibody. Additional testing (eg, RhD, malaria, CMV, Tcruzi) shall be subject to the characteristics of the tissue or cells donated or donor's travel and exposure history. In partner donation the clinician shall determine and document, based on the patient's medical history and therapeutic indications, the justification for the donation and its safety for the recipient and any child(ren) that might result. In case of nonpartner donation, age, health and medical history of the donor shall be ascertained by a questionnaire and personal interview. Genetic screening for autosomal recessive genes known to be prevalent in the donor's ethnic background and assessment of the risk of transmission of inherited conditions known to be present in the family shall be carried out as well.

\section{What type of family history?}

Generally agreed principles on the extent and quality of the family history asked from a prospective donor do not exist. A three-generation pedigree would provide enhanced information for planning relevant testing. ${ }^{129}$ Family history may contain monogenic, chromosomal or multifactorial diseases including malformations in many generations. There are no means to check whether the given family history is true. In genetic counselling it is well known that, in some populations, diseases are not very openly discussed within the extended family and thus family history may not be fully known. Many clinics do not accept donors if hereditary (including multifactorial) diseases have occurred among first-degree relatives. See also donor- specific requirements of EU Tissue Directive (Appendix A).

\section{Choosing characteristics}

Many clinics enable some selection regarding the appearance of the donor, such as skin colour, height, hair, eye colour, based on his/her resemblance with the prospective parent(s). One generally accepted indication for selection of a donor is the couple's desire to have full siblings, in other words, if donor gamete has already been used to assist conception for the couple's first child, the same donor can be used to the following assisted conceptions in order to try to have some similar characteristics.

The selection mechanism could be very delicate and very comprehensive, and is applied in particular in the USA. In Europe, the attitudes are cautious with respect to choosing characteristics.

\section{What type of counselling is provided to counter- selected people?}

If an individual is counter-selected and told that his/her gametes cannot be accepted for donation owing to hereditary diseases in the family, the message might be that he/she should not have own children either. Therefore, detailed genetic counselling should accompany counter-selection to avoid unnecessary misinterpretations and to give accurate information on the disease, reproductive choices in the future as well as to provide psychological support and counselling to family members.

\section{Carriers? Homozygosity? Limitations to use the same donor?}

Not all carriers can be detected by family history alone. The donor himself/herself may learn about his/her severe, for instance, late onset, disease tens of years after the gamete was used. The question may arise as to whether genetic screening should be applied when choosing gamete/ embryo donors and whether repeated use of the same donor in a small community, narrowing of the gene pool, might increase risk for certain autosomal recessive conditions in future generations.

The couple may consider a sister or brother as a donor. However, in case of genetic infertility like Fragile X, CF or chromosomal translocation, it is possible that he or she is also a carrier, which should be tested to avoid the risk of a serious disease in the offspring. However, this could create a conflict: brother or sister might like to donate gametes, but may not like to know his/her carrier status.

According to the IFFS Surveillance 04, countries tend to limit the use of the same donor's gametes from five to the maximum of 10 children. ${ }^{25}$ However, according to an opinion on the basis of relevant studies, these limits do not seem to be based on any valid population genetics argument. ${ }^{130}$ However, after an incident in the Netherlands, where 18 children were conceived from sperm of one donor, who later developed a serious hereditary disease, the Netherlands decided to reduce the previous limit of 25 offspring, and now the donors' opinions are asked. The practice so far shows that donors usually prefer to have approximately five children. This may also relate to whether the donation is anonymous or nonanonymous.

Knowledge of having a large number of children or halfsiblings may also be a psychological problem for some of the children and donors (www.cryos.dk). In Cryos' opinion, the inbreeding risk criterion is not the total number of children born, but the number of children created via 
donor insemination (DI) per capita in each community. In cosmopolitan communities, a larger number of offspring from the same donor would not create such consequences as in smaller communities. Legal limits differ greatly from, for instance, 5 in South Africa (total population approx. $45000000)$ to 1 per 32.000 in the USA. If the limit is set too low, it will result in a price increase and/or a reduction in the availability of DI, leading to reproductive tourism and the Grey Market (www.cryos.dk). Several studies have calculated the risk of consanguinity and have concluded that the risk is very small. ${ }^{131}$

It has been argued that when limiting the number of children per donor, it should be noted whether all or part of the children have been born to the same mother, as well as the geographic origin of the recipients. It is quite different to have had five children with five women from different countries than with five women from the same middle-sized town.

\section{Embryo donation}

Embryo donation has become an acceptable practice in many countries and may be used by a couple if both partners lack gametes or have gametic failures, or after several unsuccessful attempts of ART. ${ }^{132}$ It may also be considered in parents at high risk of having a child with a genetic disorder.

Embryo donation requires special attention, as donated embryos usually originate from the parents who have been subject to IVF treatment themselves. Consequently, it cannot be excluded that these supernumerary embryos are at increased risk of carrying a known or unknown genetic defect relating to parental infertility. ${ }^{132}$ The Genetics Commission of the French Federation of CECOS has recommended that embryos with a well-defined genetic risk for the future child should not be made available for donation. The recipient couples should be clearly informed of the risks. ${ }^{132}$

\section{Counselling in the relation of genetics and art Definition and purpose of the counselling}

Counselling means the provision of objective information from the counsellor and its interpretation by a patient' ${ }^{133}$ The purpose of counselling is, among other things, to enable patients to make informed decisions, and also find and accept other reproductive options, such as adoption, gamete donation and refraining from offspring. Counselling has also an important goal in helping patients to develop coping strategies to sustain and accompany persons in adverse circumstances or after difficult or traumatic experience. Counselling aims at empowering counselees for decision-making that reflects their values and not those of the counsellor. In genetic counselling situations, counselling often extends to family members and relatives.
Each act of counselling consists of a contract between counsellor and counselee, and therefore it is of great importance to clarify the contract, to formulate it as precisely as possible and also to revise it regularly, because the attitudes and goals of the patients may change during the course of time, and other solutions than ART may become equally or more attractive (eg, adoption). Also, new techniques or cures may have become available.

\section{Reproductive counselling}

Reproductive counselling means consultation with a fertility professional during which the patient is given information on different forms of fertility treatment, their advantages and disadvantages as well as risks, and any diagnostic tests that need to be conducted to identify the cause of infertility. If needed, patients should be referred to a psychologist or a psychiatrist.

Ample time should be devoted to reproductive counselling, as it is an essential part of planning of ART. Fertility professionals should have education on reproductive counselling in their training. For different counselling situations see ESHRE Guidelines for Counselling in Infertility (Guidelines for Counselling in Infertility Contents. ESHRE Monogr, 2002:1. Oxford Journals).

\section{Genetic counselling}

Genetic counselling is a communication process that deals with the occurrence, or risk of occurrence, of a genetic disorder in the family. The process involves an attempt by appropriately trained person(s) to help the individual or the family to (i) understand the medical facts of the disorder; (ii) appreciate how heredity contributes to the disorder and the risk of recurrence in specified relatives; (iii) understand the options of dealing with the disorder; (iv) choose the course of action which seems appropriate to them in the view of their risk and their family goals and act in accordance with that decision; and (v) make the best possible adjustment to the disorder in an affected family member and/or to the risk of recurrence of that disorder. ${ }^{134}$

As genetic counselling is extremely important, it is worrying that one-third of the genetic testing centres surveyed (EMQN report) were not linked to clinical genetics services. ${ }^{135}$ The importance of an appropriate, nondirective genetic counselling is emphasised, for instance, in Article 11 of the International Declaration of Human Genetic Data of 2003. ESHG has addressed genetic counselling in its previous review 'Provision of genetic services in Europe: Current Practices and issues'. ${ }^{134}$

A multidisciplinary expert group in the field of law, philosophy, ethics and medicine, invited by the European Commission, published in 2004 their '25 Recommendations on the ethical, legal and social implications of genetic testing'. In this document genetic counselling is considered an essential requirement for genetic tests and the importance of qualified professionals, education and 
standards is stressed. The need for Europe-wide general standards for fundamental principles of genetic counselling by medical professionals is thus acknowledged.

Patients value counselling and demand accurate information to avoid unnecessary fears as well as false optimism. ${ }^{114}$ Counselling has been noted to provide help in areas of psychological assistance, technical explanations and discussing relationships. ${ }^{136}$ At best, genetic counsellors are sensitive also for the burden of an infertility diagnosis and the emotional impact - including anxiety and ethical questions - of different treatment options. If needed, patients should be referred to a psychologist or a psychiatrist for emotional support or formal psychotherapy in which these emotional issues can adequately been addressed.

\section{Autonomy}

The ideal of modern genetic counselling is not only to avoid a directive approach (whenever suitable) but also to concentrate on the medical, psychological and social circumstances so that couples can make decisions that are appropriate for them.

In the preparatory phase, the genetic counsellor should first of all let the couple express their desires and expectations. Some patients may not want to make an autonomous decision, and some of them have already made their decision and really want the counsellor to validate it, etc.

Autonomy is problematic, though, because patients may not have the capacity to deal with excess information. Moreover, the specific context of ART and genetics needs specific modified ethics doing justice to the responsibilities and autonomy of professionals involved.

Traditional ethics of clinical genetics, in particular the emphasis on respect for reproductive autonomy and on nondirectiveness (ND), has been developed in the general context of genetic counselling in various situations. In the context of ART, however, the doctor involved has his own responsibility to avoid serious harms to future children (and his own professional autonomy), and because he is directly involved in reproductive decision-making/reproduction, he cannot ignore the consequences of his acts (professional duty of care). In view of this, it has been suggested to be justified

(a) to give access to IVF/ICSI to couples at high risk of having an affected child only on the condition that they accept PGD (in order to eliminate/reduce this risk) and

(b) to accept a shift in the locus of decision-making after PGD from the patient to the doctor. It is indeed the doctor who should decide which embryo(s) will be implanted. (The situation is, from a moral point of view, different in comparison with the traditional context of PND, where the mother has decision-making authority ${ }^{26}$ ).

\section{Challenges of counselling}

Counselling is challenging because counsellors and counselees are shown to value different information and hence understand and remember it differently. The high degree of uncertainty relating to genetic counselling is in direct contrast to the needs of clients. ${ }^{137}$ Thought should also be given to the potential conflict between legal requirements to disclose all information and the need of some individuals to avoid information ('a right not to know'). One solution proposed to avoid this conflict is to inform the patient in the initial stage of counselling that he or she can decide whether or not to be told about the subject under discussion in more detail. ${ }^{137}$

The increase of crossborder-assisted reproduction services disturbs the principles of counselling owing to cultural and language problems and might create a need to agree on uniform goals and practices of the counselling, including the problem of directiveness $v s$ ND. In addition, uniform practices of the consent process should be agreed upon.

\section{ND vs neutrality}

Nondirective genetic counselling has been defined as the provision of accurate, full, and unbiased information in an empathic relationship that offers guidance and helps people work through their own decisions' ${ }^{29}$ Applied to genetic counselling, 'the non-directiveness describes procedures that aim at promoting the autonomy and selfdirectedness of the client'. ${ }^{138}$ It was in 1942 that Carl Rogers presented the term ND to describe his personal approach to psychotherapy and renamed it later as clientcentred therapy in $1951 .^{139,140}$ The origins of the term lay hence in psychoanalysis and psychotherapy.

$\mathrm{ND}$ is an active strategy requiring quality counselling skills and assumes that most clients have the ability to make their own decisions. ${ }^{138}$ Because genetic counselling inevitably entails tensions between conflicting values, a professional should be aware of them. ${ }^{96}$ The ND in the sense of not influencing the clients may be considered as negative and may remove opportunities for reflection from clients who might find broader social and emotional issues helpful in decision-making. ${ }^{96}$

The term neutrality should not be used as a synonym of ND, because it lacks an essential component of genetic counselling, namely the empathy and involvement of the counsellor. $^{141}$

\section{Shared decision-making}

The nondirective decision-making approach may sometimes prove unattainable, especially in situations where there is significant ambivalence about the decision. 'Shared decision-making' can then provide a complementary approach when trying to balance the tensions between evidence-based guidance and the need to respect patient choice. ${ }^{142}$ In particular, in PGD, where the ambivalence concerning the decision may be high, a model of shared 
decision-making is probably more appropriate. ${ }^{115}$ This approach may balance chances for success and advantages with risk, burden and other disadvantages. ${ }^{75}$

\section{Risk communication/language of uncertainty}

Probability information is essential in genetic counselling. The meaning or interpretation of verbal uncertainties can easily be influenced by characteristics of the context (eg, desirability of an event, its perceived seriousness, its baserate). The way information is presented (eg, negative $v s$ positive framing; loss $v s$ gain framing; numerical $v s$ verbal risk information; relative $v s$ absolute risks; etc) can have significant effects to the results. These different presentation models leave space for manipulation of the goal of risk communication. ${ }^{143}$ People seem to prefer to receive probabilities in a numerical mode, even though they would handle uncertainty verbally. ${ }^{141}$ The vagueness of verbal terms does not make verbal expression of uncertainty inadequate in genetic counselling.

\section{Understanding}

There is a need to focus more on what the patients really understand. It is doubtful whether the couples internalise the risks of treatments, the potential of disappointments, etc. Counsellors and counselees are shown to value different information. A third of the information that the researchers regarded as key points were not recalled by the counselees some weeks after the consultation. ${ }^{35}$ A French survey examined what pregnant women understood from information given during prenatal testing for Down's syndrome. The findings showed that roughly half of the participants had misunderstood or ignored the information presented. ${ }^{144}$

\section{Counselling and PGD}

Genetic counselling in the context of PGD has to take care of both communication and coping strategies. Women and men considering the procedures of PGD have almost always experienced difficult situations and may not have been offered adequate counselling to cope with their situation. Being told that one is carrying a gene or a chromosomal aberration that may cause a serious condition for oneself or one's offspring is a traumatic experience to most people. It may disrupt personal identity and provoke self-doubts or feelings of inappropriateness and guilt. Nevertheless, parents should be told that all people are carriers of several mutations, which only seldom become obvious.

Multiple pregnancy losses are equally disruptive, disempowering and traumatic experiences. Moreover, other family members may need to be informed of their risks. All this may adversely affect the couple's relationship or the relationships within families. Only infrequently will this suffering be communicated spontaneously by one of the partners or by the couple. Therefore, it is important to asses carefully the couples' psychological state, the stability of their relationship and their coping strategies as well as support structures and social networks before offering PGD, as the procedure in itself is likely to put a considerable strain on the couple, not least because of the low success rate. Couple experiencing a failed PGD cycle also should be offered professional support to help in developing coping strategies. Genetic counsellors have therefore an important diagnostic responsibility to differentiate between 'normal' and 'pathological' grief reactions in patients, partners and couples. If necessary, patients should be offered appropriate help or be referred to a psychiatrist/psychologist with experience in the field.

Genetic counselling before PGD for late-onset disease should be given by a clinical geneticist or a counsellor who has experience with predictive testing for the specific lateonset disease and who is prepared to discuss the pros and the cons of all options for prenatal and preimplantation testing with the prospective parents.

ESHRE's best practice guidelines for PGD and PGS of 2005 provide specific guidance on counselling, informed consent, patient inclusion/exclusion criteria, various techniques and diagnosis, etc. ${ }^{77}$ The guidelines recommend the following steps in the PGD procedure for all couples at high genetic risk owing to structural chromosome abnormalities or monogenic diseases: a clinical geneticist or genetic counsellor first counsel potential parents to discuss the use of PGD for their particular disorder; thereafter, a clinical fertility specialist should see and evaluate the couple as for routine IVF. ${ }^{77}$ ESHRE advises to test only embryos of couples who were ready for the results and accept all the implications of the test. ${ }^{76}$

Indications for PGD and PND are often rather similar, but the efficiency of the methods as well as the consequences of the test and treatment may be different. If couples are considering PND owing to a risk of certain disease, they should be informed also of the possible availability of PGD. With respect to PGD, couples should be informed of residual risks and the uncertainty, which may relate to the PGD techniques and be offered the possibility of PND for confirmation. Couples who do not want to experience pregnancy under uncertainty and wait till PND with a possibility of a pregnancy termination may prefer PGD. However, PND is used by some couples who want information about their fetus, but who will not contemplate a termination. PGD is being used for a growing list of conditions where PND would rarely be used, for example, late-onset cancer syndromes, or not used at all, for example, HLA matching.

Prediagnosis genetic counselling before PGD A genetic counselling session should be characterised by openness for discussion. The counsellor should give the couple a general understanding of the principles of ART (eg, ovarian stimulation and IVF/ICS and potential risks). Limitations of 
the PGD method should be brought up. Important points to be expressly communicated are the aim, the type and the reliability of the test; the possibility of obtaining unexpected or false results; explanation of nature and severity of the inherited disorder and its recurrence risk; the theoretical risks involved in the test (eg, possible longterm negative effects, which are at present unknown); possible psychophysical repercussions; reproductive options and alternatives to PGD. The risk that embryos may not be suitable for biopsy, may not survive biopsy or may not be able to be diagnosed after biopsy ${ }^{76}$ or may not be suitable for transfer owing to genetic status or poor embryo quality also has to be discussed.

Postdiagnosis genetic counselling after PGD Appropriate genetic counselling includes also postdiagnosis counselling to verify that the information given is comprehensible and understood; to interpret the results and evaluate and discuss the consequences and available options; to provide discussion for decision-making; to inform about possible psychological impact; and ensure adequate means of support, in particular if the treatments fails. This implies that a psychiatrist or psychologist with interest and experience in the field should be part of the team and that the centre knows to whom patients can be referred to.

\section{Research on counselling}

Marteau et $a l^{145}$ studied the association between the outcomes of pregnancies diagnosed with $\mathrm{KS}$ and the specialty of the health professional providing pre- and postdiagnostic counselling. According to the study, there is an association between whether or not a woman terminates a pregnancy affected by an unfamiliar fetal anomaly and the professional background of the health professional providing postdiagnostic counselling. The study raises the possibility that decisions made after the diagnosis of a foetal abnormality may reflect the knowledge or values of health professionals: the affected pregnancy was more likely to continue when postdiagnosis counselling involved only a geneticist. In addition, remarkable differences between European countries were observed. ${ }^{145}$ More research is needed on counselling and in particular on framing effects of different ways of presenting risks; heuristic and biases in risk perceptions; emotional and motivational factors that influence risk perception and interpretation; and means for avoiding undesired effects. ${ }^{141,143}$ In addition, conversational or discourse analysis of genetic counselling in the field of ART might reveal unrecognised problems and tensions in these situations.

\section{Potential adverse effects of ART Introduction}

The question about possible adverse effects of ART to the child or to the mother is still controversial. ${ }^{13}$ Risks may relate either to the techniques (eg, biopsy for PGD or microinjection in ICSI), drugs used during the treatment, culture media during in vitro processes or underlying cause of infertility per se, ${ }^{146}$ maternal age, etc.

Many studies on adverse outcomes have been performed, but the methods and patient materials are often not comparable and conclusions have been diverse. While mentioning, for example, the technique used and maternal age as background information, most studies have not included the initial reason for using ART. Moreover, the target groups have been too small to give accurate data of rare conditions. For methodological challenges see later section 'Methodological challenges of research of the effects of ART'.

Despite the ambiguity of the studies, recent reviews of studies conclude, however, that children born following ART are at increased risk of birth defects compared with spontaneous conceptions. ${ }^{13,15}$

\section{Multiple gestations}

Multiple gestations are a major problem with ART and risky for both the woman and the fetus. ${ }^{11}$ Risks to the mother include inter alia hypertensive disorders, pre-eclampsia, thromboembolism, urinary tract infection, anaemia and vaginal-uterine haemorrhage (placental abruption, placenta previa), and fluid overload in association with parental tocolysis. ${ }^{147}$ Risk of stillbirth and early postnatal deaths are increased. Obstetric, neonatal and long-term consequences of multiple gestations for the health of ART children are enormous, resulting mostly from premature birth and low birth weight. ${ }^{147}$ Cerebral palsy is one of the most significant neurological impairments associated with multiple births, and increases in line with the number of fetuses. $^{147}$

ESHRE has set the reduction of the multiple pregnancy rates as a high priority for assisted reproduction programmes in $2000 .^{20}$ Moreover, Scandinavian experts have debated for new strategies to avoid multiple pregnancies and suggested that (i) clinics should endeavour to implement one-embryo transfers; (ii) two-embryo transfer should be used only for those women who are at low risk of multiple pregnancy; and (iii) ART results should be presented as 'birth per embryo transferred' and this term should be given the status of the criterion of ART excellence'. ${ }^{148}$ If a singleton pregnancy is the goal of the infertility treatment, then a multiple gestation could be viewed as a complication of the treatment. ${ }^{149}$

One of the most promising practices to reduce twin pregnancies has been elective single-embryo transfer (eSET). ${ }^{72,73,150,151}$ Cryopreservation is essential in conjunction with this application. ${ }^{72}$ An important issue is how to select patients suitable for eSET and embryos with a high putative implantation potential. The typical patient suitable for eSET is young (aged $<36$ years) and in her first or second IVF/ICSI trial. Embryo selection is performed using 
one or a combination of embryo characteristics. ${ }^{72,73,151,152}$ In Sweden, where a practise of single-embryo transfer was put into effect in January 2003, the rate of SET is now approaching $70 \%$, with the rate of multiple pregnancies going below $10 \%{ }^{72}$ Although PGS does not seem beneficial in an environment of transfer of unrestricted numbers of embryos, it may prove to be of more value in singleembryo transfer. ${ }^{9}$

Multiple gestations may not be totally preventable, because some techniques (eg, assisted hatching) have been associated with monozygotic twinning. ${ }^{153,154}$

\section{Other risks to the woman}

Among the patients who become pregnant after assisted conception, around $4 \%$ of the pregnancies will be ectopic. The embryos migrate to the ostial ends of the tubes after transfer, or they may inadvertently be placed there when they are transferred. Heterotopic pregnancy (a multiple pregnancy with one embryo in uterus and one in the tube) is extremely rare with natural conception, but the rate may be as high as $1 \%$ in assisted conception. Careful ultrasound monitoring after assisted conception detects these risks. ${ }^{155}$

Oocyte procurement also produces a risk to the woman, because she has to undergo superovulation and oocyte retrieval, which involve the risks of ovarian hyperstimulation syndrome. In particular PGD demands many oocytes. The risk of hyperstimulation could be avoided by using in vitro maturation (IVM) of oocytes when treating infertility. $^{156}$

The discussion of possible long-term effects of the hormones and other medication to woman's health has initiated some studies to examine the connection between ovulation-inducing drugs and gynaecologic cancers and breast cancer. A review article ${ }^{31}$ has analysed these studies and while not finding reasons for concern demands, however, prospective, larger-cohort, multicentre studies with longer follow-up periods. The article notes further that gynaecologic cancers may be overdiagnosed among infertile women, because of the careful follow-up during treatments or because an already existing cancer might be stimulated by the hormonal changes. According to a recent French study, infertility treatments do not increase the incidence of a breast cancer. ${ }^{157}$ More research is needed for ovarian cancer risk, despite some reassuring results. ${ }^{158}$

\section{Adverse effects to the child}

Diverse studies Several studies and large reviews have tried to evaluate the risk of adverse effects of ART to the child, but the results are partly controversial. As mentioned earlier in section 'Multiple gestations', multiple gestations constitute clearly the major risk to the child. Many of the other causes behind adverse outcomes are unknown, although studies have suggested correlations with ART and in particular ICSI.
Hansen et al $^{159}$ concluded that infants conceived with the use of ICSI or IVF have a twice as high risk of a major birth defect as naturally conceived infants, even though the absolute numbers are quite low.

It is not clear why also IVF singletons seem to do worse than their naturally conceived counterparts in terms of perinatal outcomes. ${ }^{160}$ Data on obstetric outcome and neurological sequelae, ${ }^{161}$ obtained by crosslinkage with the Danish national registries on 9557 born IVF/ICSI children, 642 survivors of a vanishing co-twin, 5237 singletons from single gestations and 3678 twins from twin gestations originating from clinical pregnancies detected by transvaginal sonography in gestational week eight, showed significantly increased risk of preterm birth, low birth weight and a tendency towards increased risk of cerebral palsy in survivors of a vanishing twin (A Pinborg, personal communication). Thus, vanishing twins and poorer outcomes in the surviving co-twin might explain some of the difference between IVF and naturally conceived singletons and may stimulate towards a general policy of singleembryo transfer. A comparison of medical records on malformations in 1139 infants consisting of 736 singletons, 200 sets of twins and one set of triplets born after ICSI with all births in Sweden using data from the Swedish Medical Birth Registry and the Registry of Congenital Malformations, ${ }^{162}$ showed that the increased rate of congenital malformations mainly was a result of a high rate of multiple births. These data illustrate that factors (double-embryo transfer) associated with ART rather than the techniques themselves may lead to poorer perinatal outcomes, and increased prevalence of malformations.

One Finnish study concluded that neonatal outcome after IVF is worse than in the general population with similar maternal age, parity and social standing, mainly owing to the large proportion of multifetal births. The higher prevalence of heart malformations did not, however, solely arise from multiplicity but also from other unknown causes. ${ }^{163}$

A thorough US examination ${ }^{164}$ of all relevant articles (2444 articles, out of which 169 were found eligible for review) concluded that the evidence generally was suggestive of no association between ART and the rates of the serious malformations. According to the review, there is: (i) enough evidence that ART is associated with some adverse neonatal outcomes (low birth weight, perinatal mortality, premature birth also in singleton births); (ii) suggestive evidence of an association with ART and some congenital conditions (Angelman, Beckwith-Wiedemann); and (iii) suggestive evidence of no association with paediatric cancer and adverse psychosocial and developmental outcomes.

A new international multicentre cohort study ${ }^{12}$ was designed to examine both birth defects and mental development of children born after ICSI, IVF and natural conception, consisting altogether of 1500 children from 
several European countries, who were followed up to age 5. The study concluded that singleton IVF and ICSI children were more likely to need healthcare resources than singleton babies born after natural conception. Assessment in general was reassuring, excluding higher rates of congenital abnormalities among ICSI children.

A large Danish study observed equal frequencies of childhood cancers, mental diseases, congenital syndromes and developmental disturbances in 442349 singleton nonIVF and 6052 IVF children, ${ }^{165}$ and did not find support for previous suggestion of an association between ART and retinoblastoma. ${ }^{166}$ Lidegaard and colleagues observed, however, an $80 \%$ increased risk of cerebral palsy among IVF children.

A Swedish population-based study on possible excess of congenital malformations came to the conclusion that there is an increased risk for congenital malformations after IVF, regardless of the technique used, and is mainly owing to parental characteristics. ${ }^{62}$

Epigenetics Evidence has been presented from animal systems that in vitro embryo culture and embryo manipulation affect epigenetic mechanisms such as DNA methylation and imprinting. ${ }^{167}$ The safety of ART at the epigenetic level has not been well studied. Epigenetics refers to the phenomena where modifications of DNA methylation and/or chromatin structure underlie changes in gene expression and phenotype characteristics. Epigenetics cover a broad range of effects: DNA methylation, imprinting, RNA silencing, covalent modifications of histones and remodelling by other chromatin-associated complexes. Disturbance of epigenetic reprogramming may influence gene expression and phenotype characteristics. Moreover, epigenetic changes that occur shortly after fertilisation, before specification of the germ line, will involve both somatic cells and germline cells, and may lead to inheritance of an epigenetic trait resulting in transgenerational phenotypes. An important property that distinguishes epigenetic modifications from genetic modifications or mutations is their potential reversibility. ${ }^{167}$

Environmental factors like superovulation and culture medium may interfere with overall methylation reprogramming and embryonic development. A higher percentage of aberrant genome-wide methylation patterns were observed in two-cell embryos from superovulated female mice and when specific synthetic media were used. ${ }^{168}$ DNA methylation plays also an important role in the mechanism of genomic imprinting.

Imprinting Imprinted genes exhibit a parent-of-origin specific pattern of expression. Imprinted genes play key roles in embryonic growth and behavioural development and they are also involved in carcinogenesis. ${ }^{167}$ Such genes have been shown to be targets of molecular defects in particular genetic syndromes such as Beckwith-
Wiedemann (BWS) and Angelman syndrome. Several recent studies suggest a possible link between ART and genomic imprinting disorders. ${ }^{169-175}$ For instance, the data resulting from studies of Marques et al ${ }^{175}$ suggest an association between abnormal genetic imprinting and hypospermatogenesis, and that spermatozoa from oligozoospermic patients carry a raised risk of transmission of imprinting errors. ${ }^{175}$ Moreover, a recent investigation suggests that superovulation may be associated with defects of genetic imprinting. ${ }^{16}$

BWS is an overgrowth disorder resulting from mutations or epimutations affecting imprinted genes on chromosome 11p15.5. Angelman syndrome is characterised by severe mental and motor retardation, lack of speech and a happy appearance and is linked with a loss of function of the maternal allele of UBE3A on chromosome 15 . The mechanism is often imprinting. ${ }^{176}$

Subfertility per se may be associated with an increased risk of conceiving a child with an imprinting defect, meaning that some couples may have a genetic defect which predisposes to subfertility, and which also increases the risk of an imprinting defect in the child. Moreover, superovulation rather than ICSI may further increase the risk of conceiving a child with an imprinting defect (B Horsthemke in an interview, see). ${ }^{16,164}$

Nevertheless, a recent large-scale national follow-up study in Danish children born after IVF did not reveal any increased risk of imprinting diseases. ${ }^{165}$ Accordingly, Danish register data do not support reports of an increased risk of imprinting diseases after IVF.

ART includes the isolation, handling and culture of gametes and early embryos at times when imprinted genes are likely to be particularly vulnerable to external influences. Amino-acid concentrations, folate concentrations and serum in the culture media can affect gene expression. Additionally, it has been noted that the process of extended culture in mice (eg, permitting extended embryo development before transfer) can cause imprinting problems leading to abnormal development. ${ }^{172,177}$ Poor culture conditions may also influence the human embryo, but this has not been studied. Consequently, a need for studies on the effects of the culture media was stressed in the workshop in Sevilla.

Evidence of sex-specific differences in imprint acquisition suggests that male and female germ cells may be susceptible to perturbations in imprinted genes at specific prenatal and postnatal stages. Imprints acquired first during gametogenesis must be maintained during preimplantation development when reprogramming of the overall genome occurs. The understanding of genomic imprinting has been developed, including the mechanisms and timing of imprint erasure, acquisition and maintenance during germ cell development and early embryogenesis, as well as the implications of this research for future epigenetic studies in reproduction and ART. ${ }^{178}$ 
One study found a clear sex-related growth difference in human blastocysts originating from ICSI, but not in blastocysts from IVF. The mechanism responsible for the findings remains unknown so far, but according to the study group's hypothesis the ICSI procedure might interfere with the process of imprinted X-inactivation. ${ }^{179}$

\section{ICSI}

Risk factors ICSI is a widely used microinjection technique, practised since 1992, and has a high success rate, particularly in cases with a male infertility factor. However, there has been concern of genetic, congenital and developmental abnormalities in children born after transfer of ICSI embryos (eg, Golombok, ${ }^{180}$ Braude and Rowell ${ }^{181}$ and Bondulle et $\mathrm{al}^{182}$ ). The use of ICSI technique may overcome natural barriers of conception. See above page 5 for more information on male infertility and a risk of conveying infertility and other diseases to the offspring. For imprinting, see previous subsection.

PGD procedures using PCR require ICSI for technical reasons to avoid DNA contamination, whereas conventional IVF may be utilised for PGD where FISH is used. Some opinions are in favour of adding PGD to the ICSI procedure in case testicular spermatozoa is used owing to the risk of aneuploidy presenting in the offspring $\left(\mathrm{WHO}^{18}\right.$, p 387).

In France, the French National Consultative Ethics Committee for Health and Life Sciences (Le Comité consultatif d'éthique national pour les sciences de la vie et de la santé, CCNE) ${ }^{183,184}$ has addressed the issue of ICSI and referred to the considerable experience of the AZ-VUB in Brussels. ${ }^{185}$ According to CCNE, the following information was obtained regarding the studied risks, which fall into two main categories:

(a) Risks linked to the ICSI method itself: For example, there is the risk of introducing foreign material into the oocyte (toxin, virus, DNA, particles, etc) or of a trauma to the oocyte caused by the perforation. The literature does not so far provide any publication confirming or refuting such fears for humans. A recent study on monkeys has, however, mentioned the possibility of lesions to the meiotic spindle. Another study evidenced the incorporation of a fragment of foreign DNA in the embryonic genome after ICSI. ${ }^{183}$

(b) Risks linked to parental factors: Male-factor infertility differs from female-factor infertility in that there is a more considerable involvement of chromosomal abnormality in its origins. Possible transmission of infertility to the child and a risk of congenital disorders exist. $^{183}$

Adverse outcomes of ICSI First 5-year follow-up studies on ICSI children's physical health ${ }^{182}$ and psychological wellbeing and cognitive development, ${ }^{186}$ published in 2004 , were mostly reassuring in stating that ICSI has not affected the children's well-being.

1. Chromosomal aberrations: Available data so far have shown that there is a small but definite increased risk of chromosomal abnormality (1.6\%) to children born after being conceived by ICSI. ${ }^{181}$ Several studies of Bonduelle and colleagues and others have shown that in ICSI children the incidence of de novo and inherited chromosomal aberrations is approximately three times higher than in the general population. ${ }^{159,187}$ Some other studies have shown a higher incidence of chromosomal abnormalities in TESE spermatozoa. ${ }^{55,69}$

2. Major malformations: A 5-year follow-up study of ICSI children found increased incidence of major congenital malformations, particularly in boys. ${ }^{12}$ It was recognised that at least $30 \%$ of congenital anomalies are missed at birth and so the higher rates of anomalies at 5 years was not considered surprising. The higher rates of genitourinary defects were suggested to reflect paternal genetic factors rather than the procedure itself. Källen et $a l^{62}$ found excess of hypospadias after ICSI compared to other IVF. For imprinting, see subsection 'Imprinting'. Also, increased risk of musculoskeletal defects and mental developmental delays has been detected. ${ }^{159}$

3. Growth and cognitive development: The potential impact of ICSI on the cognitive development of children has raised some concern. ${ }^{180}$ However, according to a newly published psychological follow-up study of 5-year-old ICSI children, ICSI does not appear to affect the psychological well-being or cognitive development at age 5 , even though some lower scores in certain performance tests, compared to a control group of naturally conceived children, were found. ${ }^{186}$

4. Several issues, which need to be further addressed in relation to different aspects of ICSI outcome have been listed, including the role of prenatal testing during ICSI pregnancies: the significance of malformations among terminated pregnancies and stillbirths; the outcome of ICSI pregnancies in cases where nonejaculated sperm has been used; the incidence of abnormalities in children after replacement of frozen-thawed ICSI embryos; and the long-term follow-up of ICSI children. ${ }^{187}$

PGD Blastomeres at the four- to 12-cell stage are not identical, but instead express different regulatory proteins. Certain patterns of fragmentation can result in the partial and nearly total loss of the regulatory proteins and the development potential of the biopsied embryo may thus be disturbed. ${ }^{85}$ Thus, removal of one or two cells from the embryo might theoretically involve a risk of impaired development of the embryo and a potential risk to the offspring.

However, one study, published in 2004, has reviewed the 12-year experience of three of the world's largest PGD centres 
and analysed the clinical outcome of PGD in USA and Italy. ${ }^{111}$ The study comprised 754 babies as a result of 4748 PGD attempts. The incidence of birth defects rate compared to that of the general population was almost equal.

The aforementioned New Zealand study noted that there are suggestions of an increased incidence of rare epigenetic disorders in babies born as a result of reproductive techniques such as IVF and ICSI, which could apply equally to babies born after PGD. ${ }^{113}$

The report of the first 5 years experience of PGD suggested that PGD is a safe and feasible technique. ${ }^{188}$ It rose, however, a question whether there is a causal relationship between biopsy procedure and the occurrence of monozygosity.

Polar body diagnosis has not been observed to have detrimental effects on children born after the procedure. ${ }^{81}$

\section{Other techniques related to ART}

- Cryopreservation might affect gene expression or lead to other molecular effects such as 'telomere shortening and replicative senescence, damage to plasma and nuclear membranes, and inappropriate chromatin condensation'. ${ }^{177}$ It has also been shown that an increased chromosomal aneuploidy rate can be found in frozenthawed embryos. ${ }^{189}$ However, Wennerholm concluded in a thorough review that cryopreservation of embryos has no apparent negative impact on perinatal outcome and early infant development. The available data do not indicate an elevated congenital malformation rate. ${ }^{162}$ The possibility of risks of cryopreservation of GV-stage or metaphase-II-stage oocytes for health, developmental potential and for predisposition to epigenetic or genetic abnormalities is still being debated (eg, Boiso et al ${ }^{190}$ and Chen et $a l^{191}$ ).

- IVM: Some patients may benefit from natural cycle IVF with in vitro matured human oocytes. ${ }^{156,192}$ However, research on the potential risks of in vitro maturation of human oocytes for the health of the oocyte and embryo is also still at its early stage. When oocytes are retrieved from the ovary for IVM before the maternal imprinting has been completed, a theoretical risk for congenital abnormalities and genetic disease owing to imprinting errors exists. Also, in vitro culture of human oocytes has been associated with the premature separation of homologous chromatids, which can lead to trisomy formation (F Pellestor, personal communication). For instance, in Italy, oocyte freezing has been imposed by the law as the only possible way to overcome the need of repeated ovarian stimulations. However, oocyte freezing is an experimental technique and possible risks are not adequately studied (some countries have banned its clinical use at the moment).

- Assisted hatching (or any manipulation of the ZP) has been associated with a higher incidence of monozygotic twinning ${ }^{154}$ and an increased risk of twins carried in the same amniotic sac, which can lead to malformation, disparities in growth and pregnancy complications. ${ }^{153}$

- Ooplasm transfer: In practice, oocyte donation is the only way to avoid passing mitochondrial disease from mother to child. The indications for preimplantation genetic treatment of mitochondrial disorders seem very remote, but one such approach, ooplasm transfer, has been discussed and even tried as a means to avoid mitochondrial disease in the fetus when the mother is a carrier of a heteroplasmic mitochondrial mutation. ${ }^{193}$ Moreover, ooplasm transfer has been used for women whose fertilised ova do not develop normally, presumably owing to a deficiency in their mitochondria. To remedy this problem at the time of fertilisation, the oocyte is injected with donor cytoplasm. The donor mitochondria could be passed on to future generations through the resulting child. The use of cytoplasmic transfer had by the year 2001 led to 30 children born worldwide, but unexpected results appeared (in two out of 30 pregnancies the embryo's karyotype was $45, \mathrm{X}$ ) and the possible risks to the physiology of the early embryo are not known. ${ }^{194,195}$ This treatment has now been disallowed in the USA by the FDA because of safety concerns. An alternative approach might be nuclear transfer into an enucleated oocyte with normal mitochondria, as abnormal mitochondria (ie, mutations) tend to overcome the normal population of the cell (J Egozcue, personal communication). Whether ooplasm transfer should be regarded as 'a genetic manipulation of an embryo', which is illegal in many countries, remains unsolved.

- Gamete retrieval before cancer treatment: In case gametes or testicular or ovarian tissue have been removed before cancer treatment and are subsequently used for IVF, there may be a risk of passing on mutated genes leading to hereditary cancer. Therefore, the possible hereditary aetiology of the cancer should be investigated.

\section{Follow-up studies}

Bonduelle et $a^{196-198}$, in particular, have performed many international follow-up studies of ICSI, but owing to the relative novelty of the technique, ongoing follow-up is needed. The ESHRE PGD Consortium will in the future focus on the data from centres that take care of the complete PGD cycle, from patient intake to transfer and intends to extend the follow-up to the babies. ${ }^{7}$

Many countries and professional organisations have stressed the need for systematic, long-term follow-up studies on the children born after ART. The problem with follow-up studies is, however, that only a minority of parents want to inform their children about the use of ART. If follow-up studies are conducted with these children issues regarding informed consent and privacy will raise. Parents should be encouraged to take part in follow-up studies of health and development of their offspring. 
Methodological challenges of research of the effects of ART

The research is challenged by multiple biases, such as confounding effects (twins vs singletons; maternal and paternal infertility; age); multiple steps (ovarian stimulation, gamete manipulation, IVF, ICSI); and techniques (ejaculated $v s$ nonejaculated sperm; IVF vs ICSI; early embryos vs cultured blastocysts). In addition, reliable results would need larger samples to demonstrate a potential increase of rare diseases (eg, the incidence of Prader-Willi or Angelman syndrome is $1 / 15000,{ }^{167}$ but ART births represent only a small percentage of all births. Moreover, future lifetime effects may be difficult to connect to ART. Transgenerational effects are so far mostly unknown and very difficult to show. Prospective, large cohort, lifelong, multigenerational multicentre studies would be extremely important.

\section{Quality and safety of procedures}

\section{Need for quality assessment for the ART process}

So far, there have not been common European rules and regulations to guarantee minimum standards. The risk of transmission of diseases and other adverse effects has raised concern, resulting in legislation and guidance at European level. EC Directive 98/79/EC on in vitro diagnostic medical devices defines the essential requirements that devices must meet before being placed on the market and obliges mainly the manufacturers. The first European initiative to common standards for procurements, preservation, processing and distribution of organs, tissues and cells was published by the Council of Europe in 2002 and was revised in 2004 (Council of Europe, 2004), but it does not concern gametes.

The quality and safety of the procedures of gamete retrieval, preservation and processing will hopefully be improved now that the European Union has in 2004 accepted the 'Tissue Directive' 2004/23/EL that will set standards of quality and safety for the donation, procurement, testing, processing, preservation, storage and distribution of gametes, when also its technical parts become approved. For further details, see Appendix A.

Quality in the ART requires involvement of clinics, laboratories and treatment procedures. Personnel should be competent, committed, well informed and educated. Attention should be paid to internal and external controls, documentation and validation. Quality of culture media is very important. A high-quality IVF clinic offers proper and optimal treatment for the patient, which increases the chance of having good quality embryos for analysis and treatment, with good and stable results.

Workshop participants in Sevilla were unanimous that European clinics should be certified or accreditated and licensing systems should be developed by professional self-regulation. Minimum quality standards should be set. Transparency and patients' rights should be secured.

\section{Professional guidelines}

The ESHG is an international professional society founded in 1967, which promotes research in basic and applied human and medical genetics and facilitates contact between all persons who share these aims. The ESHG has issued several policy reviews and recommendations, for instance, it has examined professional and scientific views on the social, ethical and legal issues that impact on the provision of genetic services in Europe, and was worried about equal accessibility and effectiveness of genetic services, quality assessment of services, professional education, multidisciplinarity and division of tasks, as well as networking. ${ }^{134}$

The main aim of the ESHRE is to promote interest in, and understanding of, reproductive biology and medicine. It does this through facilitating research and subsequent dissemination of research findings in human reproduction and embryology to the general public, scientists, clinicians and patient associations; it also works to inform politicians and policy makers throughout Europe. On a more applied level, it aims to promote improvements in clinical practice through organising teaching, training and continuing medical education activities, developing and maintaining data registries and implementing methods to improve safety and quality assurance in clinical and laboratory procedures. It has issued, for instance, best practise guidelines for clinical PGD and PGS. ${ }^{77}$ ESHRE has important subgroups, for example, the ECIT (www.ecit.org), which is planning a European-wide network for electronic data collection from ART clinics.

The IFFS is an international democratic body whose first objective is to create links among countries, peoples and cultures in the field of Human Reproduction. The IFFS aims at stimulating the quality of care and the spreading of knowledge and awareness in the field. It also wants to contribute to the standardisation of terminology and evaluation of diagnostic and therapeutic procedures in the field of reproduction. IFFS organises World Congresses, workshops and issues consensus papers and official statements in order to help national societies in their specific objectives. It has conducted a survey of the current status of ART procedures around the world. ${ }^{25}$

The PGDIS was established in 2002 and has published guidelines for good practice in PGD in October 2004. The guidelines contain consensus points of general application that promote quality laboratory practice, enabling PGD centres to offer a good clinical outcome to their patients. A variety of aspects related to a safe working system have been taken into consideration, based on the assumption that a quality programme depends on everybody's cooperation (PGDIS). 
The EMQN started in 1998 to promote quality in molecular genetic testing through the provision of external quality assessment schemes and the organisation of best practise meetings and subsequent publishing of best practise guidelines. It is an independent nonprofit organisation with a large network and has received funding from the EU under the framework FP4. Best practise guidelines on testing for individual diseases are available at the EMQN website (www.emqn.org). ${ }^{199}$ The EMQN is based at the National Genetics Reference Laboratory (Manchester), St Mary's Hospital, Manchester, The United Kingdom.

The International Clearinghouse for Birth Defects Research and Surveillance (ICBDSR) and European Registration of Congenital Anomalies (EUROCAT) follow closely ART issues, but they have not issued opinions or policies.

Liability Questions of medical responsibility usually arise in situations which involve risk taking. ${ }^{200}$ A child may be born affected after the use of ART for several reasons. To begin with, some adverse effects on the child may be connected to ART (see Chapter 8). The question may arise, whether someone is liable for a birth of an affected child in such cases. In this contex, it is extremely important that professionals pay attention to informed consent procedure, and present the anticipated risks before decision-making.

Secondly, in case of a negligent medical action, the birth of an affected child may be regarded as damage, for which parents may present claims of 'wrongful birth' against a genetic counsellor or doctor. The parents may claim that, as a result of the fault, they have been deprived of the opportunity to eliminate or terminate the pregnancy and they are burdened with a sick or handicapped child. The counsellor may have failed to inform of the risk of a genetic illness in a child; to inform about the available new techniques (eg, PGD) and/or of risks and uncertainty of the outcomes; or to carry out tests and interpret the results correctly, which would have disclosed abnormality in the fetus. ${ }^{201,202}$ Both false-positive and false-negative test result as well as false interpretation of the consequences of the result might be equally serious and lead to, for example, unintended family planning decisions, such as giving birth to an affected child or a termination of pregnancy based on misinterpretation of the results.

Also, a child might bring a claim in respect of its 'wrongful life' on the basis of his/her impaired existence or 'prenatal injury' thorough the negligence of the medical expert. Such court cases have been taken place especially in the USA. A high proportion of such cases result from laboratory errors, which are in general clearly recognisable as negligent. The claims are usually rejected on the grounds that it is not better to be dead or aborted than alive with deficiencies. $^{202}$

In the continental legal tradition, the general rules of liability require both fault (negligence) and a causal connection between the fault and the consequence. In case of negligence, one ought to have acted otherwise. The fault is in causal connection, if in its absence the damage could have been prevented. Lesions to meiotic spindle during ICSI could be considered malpractice, for instance. In case of a wrongful birth or wrongful life, the physician cannot be held responsible for causing the illness, handicap, etc; the damage to which he contributed is the birth of the affected child, to the extent of having disabled the pregnancy or its termination. Different arguments can be reasoned when considering the compensation of the damage in such cases. ${ }^{201}$ The legal tradition is different in the Anglo-Saxon world, though.

If the clinical procedures, including informed consent, have been appropriate, diligent and in accordance with professional standards, and the parents have made a voluntary autonomous decision on starting the treatments and pregnancy, grounds for successful wrongful birth actions should not exist. It has been argued that the nondirective method of clinical geneticists may protect professionals from overinvolvement with clients and perhaps also from litigation. ${ }^{96}$

\section{Research frameworks}

\section{Need for long-term monitoring}

When new techniques have emerged in the field of infertility treatment, they have often been taken into routine clinical use without appropriate research with case-control studies, etc. The chance that even the oldest and best established methods of IVF would create problems in the children in older age, for example, owing to imprinting errors, is not known, as the oldest children are not more than 27 years old. Animal studies on this subject are not feasible because of the huge differences in species in this area. The experience and knowledge about the safety of the techniques have accumulated just by using them. It has been possible to investigate the techniques only retrospectively by research groups and professional organisations like ESHRE. As the couples coming to infertility treatment are often very well aware of the possible techniques to be applied, it would be extremely difficult at present to collect control material for the existing, but not thoroughly investigated techniques in use.

Consequently, there is a clear need for more experience and data, as well as basic research in many aspects of IVF, in particular follow-up studies of long-term effects on artificially conceived children. Moreover, empirical data on the psychosocial consequences of ART, counselling and of infertility/subfertility, is lacking. Long-term monitoring of developmental and psychological outcomes for parents and offspring, and their inter-relations is hence needed.

Long-term studies are complicated by the lack of register and monitoring systems, partly owing to the parents' wish to keep the nature of conception secret from their children, 
who subsequently are not aware of the use of artificial means resulting in their birth. Also, personal data protection requirements set limits to such research requiring separate consent procedures.

In this line, 'The European IVF Monitoring (EIM) Programme' was initiated in 1999 with the aim to start a collaborative IVF data collection programme for all European countries. The data includes regional information for Europe on direct clinical results, but also on side effects, follow-up of children's well-being and also on the availability and the structure of services in the different countries. ESHRE collects, audits and publishes the data on an annual basis. Thus, the reports allow for comparisons between different countries in Europe and other regions of the world.

\section{Research needs on some of the techniques}

Among the techniques that seem particularly to require closer monitoring is ICSI, which is a widely used and clinically accepted micromanipulation technique in many countries. $^{25}$ The effect of ICSI treatment on the risk for congenital malformation should continue to be monitored. Further animal studies are needed to evaluate the safety of some procedures. Some stress the need to develop methods to detect aneuploid spermatozoa so that these can be excluded for ICSI (WHO ${ }^{18}, \mathrm{p}$ 387). The collection of information on congenital abnormalities of IVF and ICSI births needs to be more rigorous including data from neonatalogists, epidemiologists, statisticians and child development specialists (President's Council on Bioethics $(\mathrm{PCBE})^{203}$, p 73).

Reports have raised a question on whether abnormalities seen in ART are epigenetic rather than genetic, ${ }^{204}$ or whether in fact they relate to the aetiology of the infertility. ${ }^{16}$ Research is hence needed to find out the role of ART in abnormalities, that is, imprinting errors, and what specific step of ART is responsible for the connection, if any.

Also, the safety of PGD needs to be confirmed in even larger series. PGD is fairly accurate, but the possibility of misdiagnosis does exist and it has been reported. ${ }^{7}$ Further research is needed on the sensitivity, specificity and predictive value of PGD for the detection of aneuploidy (WHO, p 389). In detection of single gene defects where amplification of specific gene sequences is carried out by PCR, accurate and reliable diagnosis can be hampered by amplification failure, contamination and allele drop out, and therefore new PCR strategies are continuously being evaluated. ${ }^{205}$ The problem of mosaicism might require further research as results show that only a minority $(<35 \%)$ of human embryos derived from IVF have a normal chromosome complement in all the cells and the nucleus of one cell may not be representative of another. The effects of removing some blastomeres from the early embryo would also need closer evaluation. ${ }^{85}$ Initial evaluation has shown that PGD has no adverse consequences on early development, ${ }^{81,206}$ although it has been shown that in PGD after ICSI, a significantly higher rate of inherited chromosomal anomalies related to a higher rate of constitutional chromosomal anomalies is found, mainly in the fathers. ${ }^{197}$ An interesting research question would be whether constitutional chromosome anomalies increase the risk of de novo chromosomal anomalies. The hypothesis of a higher risk of postzygotic events as a consequence of the ICSI procedure leading to a higher proportion of chromosomal mosaicism requires further investigation. It can not be excluded that the technique itself plays a role in the formation of those abnormalities. ${ }^{207}$ In any case, ICSI is considered a highly invasive technique, and its use should be limited to strict indications. Attention may also be paid to consider circumventing the need to use ICSI when using PCR for PGD (M Pembrey, personal communication).

The latest techniques involving oocyte freezing and oocyte culture starting from ovarian biopsy are still experimental and their results should be systematically collected and followed. This applies to testicular biopsies as well.

Assisted hatching is a rather widely used micromanipulation technique, but conclusive data attesting to its usefulness are lacking. In the case of cytoplasmic transfer, foreign mitochondrial DNA seems to be maintained in the infant, which has caused some concern, although abnormalities attributable to this foreign DNA have yet to be identified. $^{25}$

The possible safety of analysing the first and/or second polar body instead of one or two cells of the embryo in the cleavage stage or later has not been proven in large studies.

\section{Further research on societal issues}

Many of the aspects of ART are not known and should hence be systematically studied. What are the ways of selecting oocyte, sperm and embryo donors? Are genetic criteria applied, and if they are, are they based on the real understanding of genetic risks or misconceptions and prejudices? Do individuals with a genetic cause for their infertility choose such ART that prevent them from transmitting the infertility to their progeny or can we foresee that genetic infertility will become more common in the future? What type of information and counselling is offered to couples and how it is understood? Which professionals are in charge in different stages? What is the impact of counselling (or the lack of it) to the decisionmaking process?

Further research on the selection of patients and on the cost-benefit ratio are needed for the evaluation of SET. ${ }^{208}$

A European wide study of ELSI (ethical, legal and social implications) of ART is needed to detect social drivers within the community as patients and services move cross borders. 


\section{Funding of the research}

Some of these issues have clear implications on public health as children born using ART constitute already a considerable part of the new generations. It is estimated that in some European countries, $5 \%$ of all births are owing to ART. ${ }^{18}$ Thus, public funding on national and EU level should be made available for studies like ELSI, for instance. At the beginning of 2005, the Commission will present its proposal on FP7, including its suggestions for thematic research priorities.

Cautious attitudes towards an embryo research prevent funding, even though it would result in more information of the first moments of human life and serve the safety of the techniques, as preclinical studies are essential for examination of the safety before introducing the techniques in clinical practise.

Collaboration between different professional groups is important to avoid parallel work. An encouraging example is cooperation between the ESHRE and the New Zealand's respective group. ${ }^{113}$

The European Group on Ethics in Science and New Developments (EGE) has in its opinion of 14 November 2000, 'Ethical aspects of human stem cell research and use', considered that stem cell research can be funded by the Framework Programme of research of the European Union, if it complies with ethical and legal requirements as defined in the programme. In the context of European pluralism, EGE leaves the issue of allowing or prohibiting the embryo research for each member state. The EGE also sees that stem cell research based on alternative sources (spare embryos, foetal tissues and adult stem cells) requires a specific Community research budget. It recommends that the EU should insist that the results of such research are widely disseminated and not hidden for reasons of commercial interest.

\section{Regulation of embryo research}

Research of embryos, children and pregnant women is strictly regulated in many countries and is also addressed in international conventions. Research on embryos is highly controversial in some countries. Nevertheless, great improvements have been achieved by research on preimplantation embryos, for example, with variations in culture media. Also much is learned about chromosomal abnormalities. $^{25}$ Embryo research may hence be considered essential for improvement of ART, but there are sensitive issues, such as procurement of research embryos; whether to use surplus embryos or embryos created on purpose; destruction of embryos; and the possibility to implant an embryo that has been a subject to research. The time limit for how long embryo culture and research in vitro is allowed needs to be defined. Generally countries seem to have adopted a 14-day rule. ${ }^{25}$

Research on embryos usually requires informed consent of the couple or donors and many countries also require a licence from a specific governing body and/or an ethical body.

Article 18 of the Convention on Human Rights and Biomedicine $^{122}$ concerning research on embryos in vitro requires adequate protection of the embryo, if the research is nationally allowed in the first place. The convention hence leaves this issue of allowing or prohibiting embryo research for the member countries to decide upon. However, according to the Convention the creation of human embryos for research purposes is absolutely prohibited, and thus only surplus embryos are allowed to be used for research.

There is an ongoing debate in many countries about the ethics of creating embryos for research purposes. Many ethicists think that both the research use on spare embryos and the creation of embryos for research purposes can be acceptable, as the moral status of these embryos is the same and the embryos are instrumentally used in both cases. ${ }^{209}$

Most European countries have prohibited the creation of human embryos for research purposes, excluding Belgium, Sweden and the UK. The following European countries allow research on stem cells from surplus embryos: Belgium, Denmark, Estonia, Finland, France, Greece, Hungary, Latvia, the Netherlands, Slovenia, Spain, Sweden, Switzerland, and the UK. ${ }^{210-213}$

A new additional protocol to the Convention on Human Rights and Biomedicine concerning biomedical research was introduced in June 2004. The protocol covers a full range of research activities in the health field involving interventions on human beings. It does not apply to research on embryos in vitro, but it does apply to research on fetuses and embryos in vivo. General provisions include that research may only be undertaken if there is no alternative of comparable effectiveness.

The CIOMS's International Ethical Guidelines for biomedical research of 2002 recommend that research protocols on pregnant women should include a plan for monitoring the outcome of the pregnancy with regard to the health of the woman and the short term as well as a long-term health of a child. ${ }^{214}$

The Charter on the Fundamental rights of the European Union was approved by the European Council in Biarritz on 14 October 2000 and was adapted to the EU Constitutional Convention in June 2004. The provisions prohibit different kinds of practices possibly related to embryo research, namely 'eugenic practices, in particular those aiming at the selection of persons and the reproductive cloning of human beings'.

\section{Public health dimension/public policy Public health care}

The concept of health includes also reproductive and sexual health according to WHO. ${ }^{2}$ The problems relating to justice and equal access to medical services are often raised 
in this context. A fair healthcare system can be regarded to provide equal access to an adequate level of health care with a reasonable reimbursement system provided by the society. It has been argued that medical reasons have priority on nonmedical reasons and nonmedical reasons of a personal nature do not qualify public funding. ${ }^{215}$ In Europe, genetic services are mainly paid by the public healthcare system, ${ }^{134}$ while public funding of infertility treatments is diversified. There are also health insurance systems, such as in Germany and Switzerland, for instance. In addition, both genetic and ART services may also be provided by the private sector at the person's own expense.

According to European Organisation of Rare Diseases (EURORDIS), ${ }^{216} 80 \%$ of rare diseases have identified genetic origins (www.eurordis.org) and concern between 3 and $4 \%$ of births. The medical community knows relatively little about rare diseases. An accurate diagnosis, if made at all, is often made very late, and support is generally poorly provided by the public health system, including lack of effective treatment. Some of the rare diseases can be prevented by PGD.

Increased funding of IVF treatment has been considered as a key factor in reducing costs related to multiple pregnancy through increased use of eSET, single-embryo transfer. $^{151}$

\section{Access to health care, reimbursement and regulatory challenges in the EU}

According to the Treaty of European Union, health services are left to national regulation, and therefore harmonisation of ART is not possible. Tissue Directive of 2004 sets requirements for safety of some of the procedures. The access to health care is affected by many factors, such as preconditions for treatment; availability and acceptability of different services and techniques; and costs and reimbursement from the public funds.

A common European approach to the right of health care and to the individual patients' rights is becoming more and more apparent. Patient's rights fall into two categories: the so-called individual rights and social rights, the latter including protection of health and access to health care. ${ }^{217}$

The right of all European citizens to health protection can be derived from the Treaty of European Union, whereas access to health care has not been explicitly mentioned in the Treaty. The Treaty enables citizens to seek health services in other member states. In several cases, the European Court of Justice has ruled that the provisions of free movement of goods and services shall not be contravened. The free movement of services concerns also health services meaning that a clinic may operate in another member state on a temporary basis, even though such operation would not be allowed by the country in question. If certain action is legal in the community level and in one member state, another member state may not prevent such action on the basis that it is illegal there. This means, for instance, that a country prohibiting abortion cannot deny access of a so-called abortion ship to its area. In case of permanent establishment of healthcare services, however, the regulation of the member state in question is to be applied. Respectively, citizens are allowed to seek PGD and other ART services in another state.

The reimbursement issues in this respect are more complicated. The cost of infertility treatment may vary considerably from one European country to another. ${ }^{126}$ The European Court of Justice has in its practice created principles concerning the reimbursement. In the case of Decker and Kohll (1998), the Court held that the home country had an obligation to reimburse a medical device and an ambulatory service obtained in another member state on the basis that it otherwise contravenes the provision of free movement. In the case of Vanbraekel (2001), the Court decided that reimbursement of hospital services shall be based on the insurance coverage in the treating country, not on the patient's home country to guarantee the free provision of services. Establishing prior national authorisation for obtaining medical services abroad are allowed, but in the case Geraets-Smits and Peerbooms (2001), the Court decided that the expression 'normal treatment' as a national precondition for obtaining authorisation shall mean normal treatment according to the state of international medical science and medical standards generally accepted at the international level. Authorisation can be refused on the ground of lack of medical necessity only if the same or equally effective treatment can be obtained without undue delay at an establishment having a contractual arrangement with the insured person's sickness insurance fund. In respect of 'undue delay', only the individual patient's medical condition should be taken into account. The case GeraetsSmits and Peerbooms is interpreted to pave the way towards harmonisation of the right to health services in Europe. ${ }^{217}$ In the case Müller-Fauré/van Riet (2003), the Court confirmed this jurisprudence. Until now, there are no known cases relating to infertility treatments in the European Court of Justice.

A consultation process performed by the Commission revealed that the Member States have different interpretations of the jurisprudence. Consequently, the situation of patients varies accordingly. Patient mobility is considered negligible according to the report (Commission staff working paper, 2003, report on the application of internal market rules to health services). To meet this controversy, the Commission has proposed a Directive on Services in the Internal Market. The Directive aims to remove, on the basis of the case law of the European Court of Justice, unjustifiable and in particular discriminatory restrictions on the freedom of establishment and the freedom to provide services for a variety of activities, including health services. It does not aim to harmonise Member States' regulation or modes of delivery of health or social services. 
The proposed Directive does not in any way interfere with the way Member States organise and finance their health and social systems. It is for Member States to decide to what extent and under what conditions private operators such as private hospitals receive funding from the public budget or the social security system (Commissions webpage, internal market, FAQ). This directive has raised many concerns about provision of health services. Nevertheless, a recent motion in 2005 has suggested leaving healthcare services outside the scope of the proposed Directive on Services.

The Commission has carried out a follow-up on patient mobility and healthcare developments in the European Union (COM (2004) 301 final) for a high-level reflection process. On 15 July 2004, the European Commissioner for Health and Consumer Protection, David Byrne, launched a reflection process on EU health policy. This process will help shape the future EU health strategy. The reflection paper 'Enabling Good Health for all' outlines Commissioner Byrne's view of the key principles that ought to guide the development of EU health policy over the coming years.

While dissimilarity in national regulation and practices among the Member States as well as crossborder treatments might stimulate an initiative for drafting uniform standards, best practice guidelines, etc, the task might be too difficult owing to European pluralism. However, the nature and consequences of ART are such that they invoke public concern and at times stimulate government intervention in some areas. The key issues are protection of human dignity; respect for the unborn child and the parental selfdetermination in the countries; freedom of research, freedom of contract and movement between European countries; and the efficiency of medical care. Consequently, international guidelines should be based on broad principles, respecting the various national social, legal and religious aspects. ${ }^{218}$

Recently, issues connected with gamete/embryo donation have been subject to vivid discussion. The conflict of interests between recipients, physicians, donors, society in general and the future children are not fully recognised in many societies. Legal regulatory intervention leads to a decrease in supply, which in turn leads to reproductive tourism and the 'Grey Market', which is made up of (unauthorised) sperm banks or private persons offering their services at a lower or nonexistent level of screening, with the risk of sexually transmitted diseases and possible legal complications (www.cryos.dk). The worldwide web provides a number of services available to those who wish to circumvent limitations of public regulation.

If society wishes to prevent or reduce free market forces, positive eugenics, low supply, higher costs, reproductive tourism and Grey Market, it should observe these mechanisms. A large international provider of donated sperm, Cryos, with a practical view on the present situation, considers it medically and ethically appropriate to have defined and agreed minimum screening standards. If the society wants to prevent positive eugenics, it should set limits with respect to the degree of genetic testing. If the recipients want a higher level of genetic testing they must do it themselves (carrier status) or by prenatal screening (www.cryos.dk).

Economic factors play a central role in the decisionmaking process concerning, for instance, diagnostic workup of the infertility, PGS, etc, as access in public sector may be limited, and a considerable part of ART is performed in the private sector. Out of an ethical point of view, one could question how to deal with economical considerations. Also, the principles of patient autonomy are, in practice, easily jeopardised by social, family and economic pressures on the woman, especially where there is a risk of remaining without a child; a severe disease in a family; or if an abnormality in the embryo or fetus is discovered. Some argue that genetic counselling should clearly be separated from public health policies. ${ }^{96}$ On the other hand, doctors are nowadays seen to have a dual responsibility ${ }^{219}$ and are expected to pay attention to social implications as well.

Crossborder treatments The main reasons for crossborder flow of gametes and patients seeking treatment abroad are limitations in access to and availability of ART including: (1) shortage of local donors (usually owing to regulations about donor identity); (2) too specific selection criteria for donors or patients for IVF; (3) restrictive regulation and practices; (4) long waiting times; (5) high costs; and (6) lack of services or expertise. It is anticipated that new European member countries will be attractive to many couples owing to easier access and a lower price for many treatments.

Crossborder flow of patients Changes in legislation or other restrictions may increase reproductive tourism. An example is the Italian law of 2004 on ART, which falls into the category 'very restrictive'. Donation of embryos and gametes is banned, as well as cryopreservation. A maximum of three oocytes can be fertilised and every embryo has to be transferred regardless of its quality or the age of the woman. PGD is absolutely prohibited. Hundreds of couples at high genetic risk have started to seek PGD abroad. ${ }^{126,220}$ Similar tendencies have been observed in other countries where PGD is banned. Also, local restrictions concerning oocyte donation and compensation for donors have invited patients to Spain, where in 2004, almost one-third of ART couples were foreign (German, Italian, British and Irish, Swiss, Portuguese and some from South America and Asia, El Pais, 24 January 2005).

Crossborder flow of sperm and oocytes In general, various countries throughout the world have difficulties 
to produce enough donors and donor gametes to meet the demand owing to restrictive regulations (especially nonanonymity, low payment, limitation of offspring). A fresh example of expected changes in the crossborder need of gametes is stated by Cryos, which is said to have recruited 50 additional sperm donors to cover the expected shortage of donor sperm when rules on donor identity changed in April 2005 in the UK. Semen donated before April 2005 from anonymous donors may be used for treatment until April 2006 in the UK.

Removal of donor anonymity has reduced sperm donations a great deal initially, for instance, in Sweden, the Netherlands and the UK. However (at least in Sweden, where anonymity was removed in 1985), a new generation of donors have come forward, who are willing to donate also nonanonymously. Also, need for sperm donation is somewhat reduced owing to the possibility today of having biological children from IVF/ICSI. The EU Tissue Directive introduces a requirement that tissues and cells must be traceable throughout the Europe, even though it is not meant to be a tool for a child to detect its biological parent. It is not yet clear how traceability will be provided, but nevertheless, the information remains existing.

\section{Perception of normality}

The concept of genetic counselling in connection with family planning has been questioned in so far as it increasingly involves the systematic selection of fetuses, and hence approaches children as consumer objects subject to quality control. The increasing need for genetic counselling can be seen as being based on the increasing number of disorders, which can be diagnosed. There is a risk that before long, the definition of fetal imperfection will come to mean any condition that can be diagnosed.

One often used argument against different screening and tests on the fetus, the embryo or sperm before birth is that selection unfairly discriminates and will lead to disrespect of people with disorders, that is, their dignity and human value would thus be questioned.

With respect to this issue, it is noteworthy that only the rare Mendelian disorders can be regarded as truly monogenic. The majority of genetic disorders result from multifactorial traits, which are believed to be the result of not only the direct effects of one or several genes but also owing to combinations of genetic and environmental factors. Furthermore, even in the case of monogenic diseases, symptoms may vary depending on how the gene is expressed. This makes decisions about the selection of the embryos or fetuses based on the intended child's future health extremely difficult. As mentioned above, the increased use of preimplantation diagnostic methods may lead to tendency to expect, and require perfect babies. Pregnancy and birth always involve uncertainty and unpredictability; a child might have a genetic problem, be damaged in uterus or at birth. The question may thus arise as to whether the future parents have capability to cope despite intense prepregnancy planning. Wrongful life and wrongful birth claims could become more common. ${ }^{202}$

In trying to create guidelines and a list of indications or diseases, there is, besides the problems of fixed lists as such, the problem of concepts. Not even the best determinations can satisfactorily resolve the problems relating to terms, such as 'essentially', 'adverse', 'severe', etc. Moreover, a severe condition may also, in the progress of medicine, later become treatable and hence be alleviated or even cured.

\section{Ethical questions \\ ART from an ethical point of view}

The faith and potential destruction of the surplus embryos resulting from ART appeals to the moral sensitivity of many people. Some arguments against ART oppose any form of technical interference in the 'natural process' of procreation. The latest arguments concern the potential weakening of the gene pool and increasing infertility in the society. In particular, PGD brings together three areas of biotechnology each of which have engendered their own ethical debates: IVF, genetic testing and PND (for the purpose of selective continuation of pregnancy, or for the establishment of an unaffected pregnancy). In making the case for the development of PGD, the contribution of PGD to the services for families threatened by genetic disease needs to be set in the broader perspective of all PND techniques and the associated genetic services. ${ }^{221}$

The central ethical questions relating to PGD are the moral and legal status of the embryo and its potential eugenic dimension. ${ }^{127,222}$ For some critics, PGD is seen as 'eugenic', because it may enable 'frivolous choices', that is, facilitate the selection of children with certain desirable physical characteristics or intelligence. ${ }^{127}$ Technologies are developing rapidly and our knowledge of genetics and the causes of genetic diseases, or even characteristics, increases all the time, which easily leads to demands for new applications.

\section{What is wrong with eugenics?}

Some of the consulted ethicists have posed the question 'What is wrong with eugenics?' and declined to see the moral wrongness of aiming to reduce the number of people with a genetic disorder. The argument of slippery slope is rather vague. The possibility of abuse is not a sufficient argument to outlaw the use of a technique. Practices that some consider as eugenics are not viewed similarly by others (eg, thalassemia carrier screening in Cyprus before a marriage licence).

The WHO has issued the following working definition of eugenics: 'A coercive policy intended to further a reproductive goal, against the rights, freedoms, and choices of individual'. ${ }^{29}$ Upon the WHO's statement, 'Under the 
above definition, knowledge-based, goal-oriented individual or family choices to have a healthy baby do not constitute eugenics', and furthermore, 'Eugenics is directed against the whole populations, whereas the work of today's clinical geneticists is directed towards individuals and families'.

On the other hand, it has been suggested that selection for nondisease genes should be allowed, even if this maintains or increases social inequality, and pose a principle of 'procreative beneficence', in which couples select the child of the possible children they could have, who is expected to have the best life, or at least as good a life as the others, based on relevant available information. The arguments used for this is that some nondisease genes affect the likelihood of leading the best life. ${ }^{223}$ This approach is rather controversial. It can be argued that creating perfect children cannot be the goal of ART and would mean practising 'euphenics', that is, the improvement of the phenotype by biological means.

Even an extensive screening of donors might de facto lead to euphenics, with or without the aim of enhancing the gene pool. One could argue, based on equality, that genetic screening of donors should not exceed what is normal in the general population before natural reproduction.

\section{Choosing characteristics}

It has been reported that parents with a certain genetic condition, such as deafness or shortness of stature, have demanded PGD for selection of embryos carrying the same mutation as them, so that the child would better integrate into the family. The IBC of UNESCO, for instance, considers this approach unethical, because it does not take into account the many lifelong and irreversible disadvantages that will burden the future person.

An UK survey by the HGC in 2001 has found public support for using genetic information to detect disabling conditions before birth, but with clear opposition to sex selection or to selection of mental and physical characteristics of children (Ref. www.servicefirst.gov.uk/2001/panel/ hgc/index.htm). For more details on sex selection, see section 'Sex selection for medical reasons'.

\section{PGD-HLA}

Choosing an embryo that may provide stem cells for an existing person, 'a saviour child', leads to ethical discussions regarding instrumentalisation and the best interests of the future child, concern over reduced genetic diversity, unnecessary destruction of embryos and moral disapproval of society. ${ }^{223,224}$ Compared with many other persons, who are conceived by accident or without any conscious thought at all, a saviour child, however, already has a reason to exist. ${ }^{105}$ Although some regard the creating of children as saviour children as acceptable, other arguments do not support the use of PGD for the selection of embryos for 'nonmedical' reasons. ${ }^{26,27}$ In particular, the faith of the embryos that are healthy per se, but not HLA compatible, needs consideration. ${ }^{225}$

This question has drawn a lot of public attention, especially in the United Kingdom over some controversial decisions made by the HFEA. ${ }^{226}$ There is considerable ethical debate concerning this issue among professional as well (eg, BMJ Autumn 2004). No clear professional guidelines exist specifically related to the use of PGD for the benefit of an existing person. A common objection is that these children would not be valued for their own existence. Some professionals suggest that in countries were PGD already is allowed, using PGD solely for choosing a HLAcompatible embryo to provide stem cells for treating an existing person should also be permitted. ${ }^{223,224}$ France, Denmark and Norway have adapted new statutes allowing PGD for HLA matching with a sibling in 2004, and Spain is preparing amendment in the law in 2005. Also, the HFEA in the UK has eased its previous strict policy. Moreover, the IBC has taken a positive attitude to HLA typing as an additional step to PGD, if it is primarily performed to avoid an affected embryo ('selection in two stages'). In contrast, the IBC considers it unethical to perform PGD with the only goal of HLA typing and selecting embryos fit for donorship 'since the embryo becomes instrumentalised for the benefit of others' ${ }^{98}$

A case report of a 6-year-old girl suffering from Fanconi anaemia, an autosomal recessive disorder, ${ }^{227}$ gives the first example of selection in two stages. PGD was directed to finding those embryos that did not have Fanconi anaemia in order to avoid another affected child, but at the same time HLA typing was used to select for those unaffected embryos that would be a match for the sibling affected by Fanconi anaemia. ${ }^{105}$

A possible criterion to determine acceptability of PGDHLA is a so-called 'postnatal' test: it is ethically acceptable to make a child for a certain reason, if it is acceptable to use an existing child for the same reason. Optimal conditions might include no cure without transplantation; high success rate of transplantation; considerable advantage of using an HLA-identical sibling compared with alternative donors; and slow progression of the disease, resulting in sufficient time to wait for the birth of the donor child. A more general discussion of this solution is needed in order to determine whether a similar approach would be justified for other conditions. ${ }^{105}$

\section{Select or protect? What is the obligation of the medical profession?}

Most societies grant physicians a considerable autonomy in deciding what is to be regarded as the best way of implementing a medical procedure. ${ }^{200}$ One of the most important norms for medical professionals to apply is the medical principle of beneficence. The principle of ND relates to genetic counselling. The future child's interest should be considered too. How to resolve these sometimes 
controversial aspects in medical profession? Especially, the principles of professional duty of care $v s$ patient autonomy may sometimes be hard to combine.

The WMA has addressed the issue of 'dual loyalty' of physicians in its new Medical Ethics Manual of 2005. Although the WMA International Code of Medical Ethics states that 'A physician shall owe his patients complete loyalty', WMA acknowledges that there may be exceptional situations where a physician might have to consider other interests as well, such as those of other patients, society, third party. This statement could be easily interpreted as to cover offspring of the patients as well. The physician's role is nowadays considered to include a more social conception. ${ }^{219}$ The ethical challenge is to decide when and how to protect the patients.

An important question in the context of reproductive genetics is whether and under which conditions medical professionals are allowed to withhold treatment in case of a high risk of having an affected child or to start treatment only if couples undergo PGD/PND? Furthermore, with respect to nonmaleficence, is a doctor allowed to deny transfer of affected embryos in case it would result in the birth of a seriously affected child? On the other hand, with respect to the patient autonomy, is it possible to let the parents decide on giving birth to a child that is known to carry some hereditary disease, which involves suffering and/or premature death? What about the decision-making authority if some milder chromosomal aberrations (XYY or $\mathrm{XXY}$ ) are found and people want to have these embryos transferred, because there are no other embryos available?

According to the ESHRE's statement, the clinic has the right to refuse participation in the reproductive project, if it considers the risk of the future child being affected as too high, despite PGD. ${ }^{76}$ This may happen, that is, in the case of PGD, when no unaffected or diagnosed embryos are available for transfer. Parents then might reconsider their intentions and require transfer of affected embryos, especially if they otherwise were faced with a situation of 'no child at all'. ESHRE PGD guidelines state that 'it is acceptable for health care providers to object conscientiously to transferring embryos that are likely to result in the birth of an affected child. In such cases, providers should consider referring the couple to colleagues who are prepared to offer transfer of such embryos' ${ }^{77}$

Some argue that the parents have an obligation to protect their future child from preventable impairments and should take this into consideration when planning a child. $^{19}$

It is a challenge for a genetic counsellor to find out what the couple really wants. The couple is under a lot of stress during the cycle and therefore may not be fully capable of evaluating the situation. Their desire for a child may overweigh a potential genetic disease in a child. ${ }^{115}$

One could also argue that it is not in the best interests of the national healthcare system and its resources to further birth of seriously ill children, nor does it accord with the medical principle of nonmaleficence. Moreover, the goal of genetic counsellors is not to guide their patients to certain decisions, but to help them to make their own decisions (ND). It has been argued that genetic counselling should clearly be separated from public health policies. ${ }^{96}$ However, as argued previously, the new understanding of the physician's role includes a social and third-party perspective as well. ${ }^{219}$

One ethical dilemma concerns patients with susceptible capability of parenthood owing to drug abuse, unstable psychological character or social conditions, risk of child abuse, or involving other risks to the well-being of a child. Furthermore, to offer ART to couples where a parent may have a terminal genetic condition and would not be around to parent the child (eg, HD) is also problematic. ASRM has addressed the issue of offspring welfare and stated that fertility specialists are allowed to consider the patients' child-rearing ability and the home conditions of the potential child, and they can select patients and withhold services, as long as such decisions are not discriminative and are based on empirical facts derived from careful inquiry. $^{228-230,70}$ This topic is highly sensitive and controversial, and although it is part of the HFE Act in the UK, it still is open to public consultation to revisit this part of the Act. There are always those who will limit the access based on individual properties of the people, but a valid method has not been found to evaluate who will be a good parent and who will not. Often those who have a high desire to have children are also likely to be good parents. ${ }^{231}$

\section{Psychological issues}

In Western society, parenthood is experienced as one of the most important role transitions in adult life for both women and men. Hence, the experience of sub- or infertility can therefore be characterised as a 'nonevent transition'. Infertility can be defined as a nonevent that alters the individuals perception of self and of the world that demands a change in assumptions or behaviour, and that may lead either to growth or to deterioration. ${ }^{232}$ The psychological impact of infertility as such cannot be underestimated especially in the context where children are being experienced as an emotional capital (see section 'Evolution of the emotional significance of children').

Apart from the psychological impact of the diagnosis of infertility, ART treatments pose emotional challenges for a couple attending treatment. The process of a cycle itself is stressful, for example, the frustration and disappointment of several unsuccessful attempts, the concern for the health of the future child and so on. The situation is uncontrollable and unpredictable. Furthermore, the presence of a genetic defect may first have been detected unexpectedly during the treatment process. It is not surprising that psychological support may be needed. 
Some fear that the donor-assisted conception may lead to dysfunctional patterns of parenting owing to the difficulties experienced by the mothers and fathers in their quest for a child, or that parents may feel or behave less positively when they are not biological parents of a child and may not fully accept the child as their own. A further issue is that the majority of adults and children conceived in this way remain unaware that the person they believe to be their father (or mother) is not their biological parent. In recent years, there has been growing unease about the secrecy that surrounds families created by DI. ${ }^{231}$

Studies of IVF families where the mother and the father are the biological parents of the child have generally found that the parents are well adjusted and have good relationships with their children. ${ }^{231}$ ART mothers did not differ from the adoptive or natural conception mothers in expressed warmth, and sensitivity towards the child nor in affection. ART mothers showed greater emotional involvement with their child, and enjoyed motherhood more than the natural conception mothers. Also, the ART fathers showed greater expressed warmth and emotional involvement than both the adoptive and natural conception fathers, and enjoyed fatherhood more than the natural conception fathers. ART children reported less criticism or rejection from both their mother and their father than the natural conception and adopted children. No differences were identified between the IVF and DI families for any of the variables relating to parenting or the psychological well-being of the child. This suggests that the absence of a genetic link between the father and the child does not interfere with the development of a positive relationship between them. ${ }^{231}$

With respect to PGD-HLA, the child may feel proud of its role in attempting to save its sibling's life. For instance, it could be considered more devastating for one's selfconcept to be told that he or she was an 'accident'. On the other hand, it could be argued that a heavy burden is placed on the donor child. The transplantation may fail and this may give the child a fundamental sense of unworthiness and deficiency and a feeling of not being able to live up to the expectations. The child may be required for further donations of blood, marrow or other organ's. Furthermore, the consent issues may be complicated. Consequently, the psychological impact of bone marrow donation among siblings should not be underestimated. $^{233}$

Children from DI may remain ignorant of their biological parents. Firstly, it has been traditionally considered as a family matter and left for parents to decide, whether they disclose the conception method to their children or not, even though the legislation would allow to detect donor identity. From a psychological point of view, it is not clear which of both positions (disclosure or nondisclosure) is better for the child and/or his parents. In clinical practice, however, it is important to confront clients with this question in order to enable them to gain insight in the meaning of the necessity to use donor material.

Secondly, some jurisdictions support anonymity of donors. This may be thought to violate a right to know one's genetic history. This is not unique, or even a new phenomenon, although, as adopted children may have the same situation and many children throughout the history have been conceived by other than their social father. Arguments relating to the emotional and social needs of a child are numerous, some in favour, some against the identification (eg, The Danish Council of Ethics ${ }^{234}$ ). Until recently, most countries' practices or laws kept the identity of both the sperm donor and the recipient confidential. However, at the time of writing this paper, many countries' decisions emphasise the child's best interest and allow the child access to some background information whether identifying or nonidentifying. Identifying information is provided, for example, in Sweden already since 1985, Switzerland in 2001, Germany, the Netherlands in 2004, Norway and UK in 2005.

\section{Scenarios for the future: main drivers Support groups needing PGD}

The quality and the amount of information to the patients on PGD and its indications, risks, etc should be increased.

In the UK, for instance, the Genetic Interest Group (GIG) is a national alliance of organisations with a membership of over 130 charities, which support children, families and individuals affected by genetic disorders, most of which are rare diseases. The GIG has strongly argued in favour of PGD, keeping PGD and research involving embryos 'central to our members' interest'. The GIG has supported the use of tissue typing in conjunction with genetic testing to enable a family to have a child who is both free of a genetic disorder and also able to be a stem cell donor to a sick sibling (GIG's submission to House of Commons Science and Technology Committee Inquiry May 2004).

\section{Future aspects of quality, safety and efficacy}

Efficacy, measured in terms of success rate, will be a main driver for the use and development of new and improved technologies. Optimal treatment implies good chance of pregnancy with a minimal risk for mother and child. Ironically, the social and psychological consequences of infertility for the couple have increased with the advancement of ART. Given the increased expectations, the higher economic and emotional cost for the couple and the greater physical demand on women, the failure of ART leads to greater psychological and social consequences for the couple than the unavailability of these technologies. ${ }^{235}$

IVM may in the future become more generally used, ${ }^{156}$ which would prevent the drawbacks related to superovulation, such as side effects and costs of the hormone therapy. 
As there is a clear link between certain genetic abnormalities and spontaneous abortions, PGS could become a solution to detect anomalies and to improve chances of successful pregnancies, especially in patients over 36 years of age.

The 24-colour FISH painting technique, known as MFISH or spectral karyotype, allows the simultaneous and distinct identification of all the chromosomes. Adaptation of CGH-array will probably modify the conception and practice of PGD and PGS. ${ }^{236}$

Some believe that CGH could boost success rate even further by allowing a complete analysis of all the chromosomes, ${ }^{99}$ but time constraints that involves embryo freezing and therefore reduces the potential of improved implantation is at present a considerable drawback of this technique. Faster techniques that obviate the need for embryo freezing are necessary to unleash the full potential of PGD. ${ }^{111}$

Besides efficacy, the standards for quality (and with it the safety) of the procedures will also drive the level of implementation of these medical procedures. International quality standards already apply to IVF laboratories, such as the ISO 15189:2003 for medical laboratories with particular requirements for quality and competence in $\mathrm{IVF}^{237}$ In Europe, the new Tissue Directive of 2004 sets minimum quality requirements (see Appendix A) and requires labs to be accredited, designated, authorised or licensed by a competent authority of the Member States not later than 7 April 2006. It is expected that 'tissue-specific' technical requirements will be included in a series of further directives in the near future. The Directive will bring a great deal of effort for hundreds of centres, but this effort will contribute substantially towards ensuring high standards of quality and safety. There is still a risk that many clinics may find new requirements hard to attain and hence availability of services might be reduced.

\section{Acceptability of genetic testing and screening}

Another important driver will be social acceptability. Generally, clinics only test or screen untreatable conditions and usually those that manifest early in life or affect children, such as spinal muscular atrophy and CF, but the trend is to enlarge the scope to diseases that may have a later onset, for example, HD. Some pressure groups have shown discontent to allowing screening for late-onset diseases or for significant disease traits, for example, FAP because they see it as a slippery slope to screening for genes with lower penetrance or those that merely increase risk, without being a guarantee that the disorder will develop, for example, mutations in cancer predisposition genes like BRCA1. ${ }^{238}$ However, others argue that having a child with inherited susceptibility to cancer could be a major source of suffering for parents and child. ${ }^{239}$

If genetic tests for nonmedical multifactorial traits such as strength, intelligence, sexual orientation or other factors become available, the impact of PGD might be important. However, such tests, with a few exceptions, are unlikely to become available. ${ }^{240,241}$ To begin with, the number of eight-cell stage embryos available for testing is limited. Secondly, PGD enables to analyse only one to two defects from single cells. Therefore, design babies are not feasible (Outi Hovatta, personel communication).

Concerns about the potential misuse of embryo screening should be addressed.

\section{Economic constraints will be heavier}

These techniques are costly and insurance coverage will be a key issue in their future development. One restriction to the availability of genetic diagnosis might arise from commercial patents, which could raise the costs too high or even totally refuse licensed use. Reimbursement takes place mostly at public level, but infertility is not included in private insurances as there is a reluctance to consider it a disability or a medical condition, but rather an elective procedure. $^{3}$ It is conceivable that health plans that do or will cover IVF might someday require PGD for selection against potentially costly diseases. ${ }^{203}$ On the other hand, widespread use of PGD, as long as not subsidised, might widen social inequalities as access to both PGD and IVF is restricted to those who can afford it.

Scenarios for genetic testing include social, technological, economic and political drivers. As medical use of genetics is seen in a positive light by citizens and patient groups, and as they are also constantly more aware of developments in that field, there is a trend that patients are acting as enlightened consumers, which may lead to demands for the use of the latest medical achievements and increased costs of health care. Four scenarios for genetic testing are presented by IPTS in view of high or low impact on genomics and favourable or poor economic outlook: (1) Rationing of New Technologies; (2) Centralised cost-benefit rationing; (3) High demand, Diverse Supply; and (4) Diverse Supply, Diverse Utility. ${ }^{135}$

\section{Possible prevention of infertility}

Trends indicate that the decline in birth rates in Europe is long lasting and shows no clear sign of levelling off. This reflects mostly the family planning decisions involving fewer children, but at the same time infertility is increasing. Couples face increasingly more trouble to get a child. Advanced maternal age, environmental factors, smoking habits, stress, etc all may affect fertility in Western countries (eg, Klonoff-Cohen ${ }^{242}$ ). Developed countries have experienced unprecedented declines in fertility rates over the last half of the 20th century with a prevalence of subfertility close to $10 \%$, mostly owing to genital track infections. It is possible that the need for infertility services will increase.

The proliferation of infertility might be somewhat retarded by promoting younger motherhood by public 
means, for example, supporting parents economically and socially, and in particular women to combine work and family. Giving more information about the negative influence of smoking, drinking, etc to fertility might bring some help. Perpetual attention should also be paid to prevent genital track infections, which often lead to infertility. Hence, the use of condoms should be promoted more intensively, even though their use is forbidden in many cultures.

\section{Evolution of cultural groups}

Cultural pressure can make a difference, mainly in PGD for sex determination. A distinction should be made between child sex selection and the so-called 'family balancing'. Sex selection is not circumscribed to developing areas; there is demographic evidence that choosing the sex of children in the US - largely by using sonography and abortion - is happening, but there is, however, no monitoring of such demographic effects. ${ }^{203}$ It is clear that the use of other technologies (ultrasonic screening and abortion) has lead to sex disparities in China and India. ${ }^{241}$ Although PGD is currently too expensive and inaccessible to be used on a wide scale for sex selection, and although PGD would affect only marginally the societal sex ratio balance, it is a potential driver that should be kept in mind.

\section{Evolution of adoption systems}

In many European countries, attitudes towards delivering an unwanted baby and then giving the baby for adoption are not very positive. Instead, early abortion is seen as more acceptable. These attitudes may change, to one direction or the other. Many infertile couples might opt for adoption if only the process were faster and easier. At the same time, some countries in the third world have adapted stricter policies concerning foreign adoption, and thus the amount of babies available for adoption may be decreasing. In consequence, adoption may become even more difficult than today.

\section{Evolution of the emotional significance of children}

In recent history, the significance of children has shifted from an 'economical' to an 'emotional' capital. Together with this shift in importance of children, the advent of oral contraceptives has created the 'illusion' of the possibility to create our own children, if we want and when we want. The development of IVF techniques - now further developed with PGD - only strengthen people in their belief that children can be created and that nothing stands in their way in doing so. This kind of reasoning and the strength of this belief are reflected in an increase of the psychological, emotional and relational burden created by the inability to conceive own children. In the near future, it will be important to further develop the availability of counsellors, psychologists or psychiatrists who can help patients to cope with the psychological, emotional and relational burden in order to help in IVF and genetic centres where PGD is being performed (P Enzlin, personal communication).

\section{Evolution of policies toward disabled people}

Some have argued that wider use of PGD or PND may lead to more discriminative attitudes towards disabled people. However, if the number of the disabled people decreases, this might theoretically lead to more resources for treatment and rehabilitation of the individuals with disabilities. Furthermore, it has been feared that ART might in fact lead to dysgenics, altering the gene pool of populations and expanding the need for infertility treatments. Nevertheless, it should be noted that PGD and PND can only take away the extra risk of the disease known to be running in the family. The population risk of genetic and congenital abnormalities remains and is not reduced by these tests. Besides, other (nongenetic) medical reasons and accidents cause the majority of disabilities.

\section{Developments in the field of stem cells}

Stem cell research is expected to be equally important for basic science, as well as for the understanding of how diseases develop, and for the development of safer and more effective drugs and other treatments. Although countries such as France, Spain and the UK have expressed their support for stem cell research, others, including Austria, Germany and Italy, have voiced opposition. These differences in opinions resulted in an 18-month freeze on EU-funded research in this area under the Sixth Framework Programme (FP6) expired at the end of last year without agreement between Member States on how to proceed. It has been left up to the Commission to steer a principled course in deciding which projects to fund. Incoming Research Commissioner Janez Potocnik has said that he would seek to set aside funds for such research under the upcoming FP7.

\section{Developments in the germline modification techniques}

At present, genetic modifications affecting the germ line are forbidden. If, however, such modification would turn out to be safe and efficient, they might be allowed and families with hereditary conditions might want to use such techniques. This would mean that, instead of PGD or PND or, on the other hand, donated gametes, genetically modified own gametes would be used.

\section{Development in donor attitudes}

One of the most significant factor decreasing donors is the past trend to remove donor anonymity in many countries.

Donated oocytes are scarce owing to the difficulties in procuring and preserving them. The procedure for this type of donation is surgically invasive and includes some risks. Donation of oocytes from women is usually not compensated. Some clinics offer 'egg-sharing' schemes, where a 
woman who needs fertility treatment receives it at a reduced price in return for donating some of her oocytes to another woman or couple. Synchronising the cycles of two women to get fresh oocytes is difficult, but would increase chances of success, since frozen oocytes do not have as good fertilisation potential as fresh do. Unpleasant situation occurs, if the donor does not become pregnant, whereas the recipient does. The use of oocytes and/or egg sharing is forbidden in some countries. The HFEA in the UK issued a public consultation ${ }^{128}$ on this and other issues, such as the regulation governing the import of gametes and the limits of oocytes per donor.

The EGE has in its opinion of 14 November 2000, 'Ethical aspects of human stem cell research and use', stressed the necessity to ensure that the demand for surplus embryos and oocyte donation does not increase the burden on women, as women who undergo fertility treatment are already subject to high psychological and physical strain (EGE 2000). In Spain, without specific regulation on oocyte donation, women receive between 600 and 900 euros to compensate time and effort provided. The oocyte donation programme has made Spain an attractive place for reproductive tourism, as mentioned earlier (El Pais, 25 January 2005).

Embryo donation, although it is widely regarded as a cost-effective use of a valuable resource, confronts a large emotional barrier to relinquishing couples that usually do not want to consider full siblings living with other families. Raising embryo donation as a possibility using educational programmes and media coverage are depicted as a potential positive influence on embryo availability. ${ }^{243}$ It is noteworthy, however, that supernumerary embryos available for donation may be at increased risk of carrying a known or unknown genetic defect related to parental infertility, as the main source of donated embryos are couples being subject to ART themselves. ${ }^{132}$

\section{Regulation}

National and international regulation may have a great impact to ART and stem cell research in the future, one way or the other. ART and research develop at a pace faster than legislation and to an unpredicted destination. Courts and authorities might be challenged with demands of licensing new interventions, but are not the right place to settle the basics of these issues. Excess bureaucracy and endless licensing policies will certainly lead to inefficiency and retard development in this field. Regulation should guarantee a satisfactory, flexible and reasonable research framework, whereas protecting research subjects and requiring good scientific results.

\section{Conclusions}

The interface between ART and genetics comprises several sensitive and important issues that affect infertile couples, families with genetic diseases, potential children, professionals in ART and genetics, health care, researchers and the society in general.

Many representatives of the European IVF clinics reported in Sevilla that almost half of their IVF patients are over 36 years old. Advanced maternal age increases not only infertility, but also the risk of sporadic chromosome anomalies and complications. Society should hence support younger motherhood in order to decrease the need for ART and complications to both mother and child.

Ample reproductive counselling should be available to all couples coming for treatments. In addition, proper counselling in all genetic testing services, including in the field of ART, is considered extremely important. At the moment, it does not seem to be provided in the best possible way. Any person involved in the procedure of counselling (whether reproductive or genetic) should be adequately trained. Moreover, a multidisciplinary approach and close cooperation between professionals would be extremely important. Centralisation of the most complex genetic counselling situations is needed.

Practice guidelines would be needed on adequate diagnostic procedures to solve aetiology of infertility in each couple. Unexplained infertility may lead to problems in pregnancy and for the future child. Moreover, the causes of possible morbidity in the child might be suggested to be a consequence of ART even when they actually relate to the cause of infertility. Thorough investigation of the reasons behind infertility is hence desirable.

The workshop participants in Sevilla had different opinions on the extent of genetic testing of donors, but felt generally that testing should not lead to selecting better genes for donor-conceived children compared to those presenting among the general population.

PGD has become widely practiced throughout the world for various indications, and it helps to restore the reproductive confidence. PGD can substantially decrease the eventual risks of passing a genetic undesired condition to the offspring. Nevertheless, its extension to some new and nonmedical indications has raised ethical concerns. PGD is an invasive and expensive technique with a rather low success rate and involves psychological stress to the couples. One disadvantage in connection with PGD is the need to use IVF/ICSI, which might lead to other risks. The complications of multiple pregnancies should be avoided by transferring only one embryo. All the aspects and risks of PGD and other options should be weighed and balanced. PGD offers thus an alternative to PND, but cannot replace it.

Although widely used, PGS is still considered as an experimental procedure and its clinical utility is not fully proven. PGS may improve the success potential of a singleembryo transfer and thus help decreasing multiple pregnancies with high risks to both mother and child. In addition, need for termination of pregnancy after PND might be reduced. 
The need to shift towards a single-embryo transfer has been stressed to avoid risks of multiple pregnancies. Potential other side effects, either related to medical procedures of ART or parental factors, need to be envisaged further, as the studies carried out so far cannot give adequate knowledge of the impact of the ART procedures. High-quality clinical research should be performed before introducing new techniques into clinical practice. More preclinical and follow-up studies are hence needed.

The information given to patients should be evidence based. The concept of success rate needs evaluation and standardisation.

Reproductive autonomy vs professional duty of care constitutes an ethical challenge for professionals. Their dual responsibility requires focusing also on the future child's interests.

Diverse levels of access and provision of services within the EU Member states has led to crossborder reproductive treatments. However, considering the pluralism in Europe and the fact that legal harmonisation is impossible and even undesirable, a general legal framework is not a solution. Instead, patients treated in different European countries should be entitled to access of the same standard of care through professional guidelines and systems of accreditation. Adequate counselling in connection with crossborder treatments should be secured as well.

Both ESHG and ESHRE feel that professional recommendations on the very sensitive interface between ART and genetics are urgently needed. Everything that is possible should not be carried out. It is clear, however, that any recommendations on this quickly developing field must be reviewed regularly.

\section{References}

1 Steptoe PC, Edwards RG: Birth after the preimplantation of a human embryo. Lancet 1978; 2: 366.

2 WHO: Reproductive Health Strategy 2004.

3 Dill S: Consumer perspectives in Current Practices and controversies in assisted reproduction. Report of a Who Meeting 2001. Geneva: WHO, 2002.

4 Handyside AH, Kontogianni EG, Hardy K, Winston RM: Pregnancies from biopsied human preimplantation embryos sexed by Y-specific DNA amplification. Nature 1990; 344: $768-$ 770.

5 Boyle KE, Vlahos N, Jarow JP: Assisted reproductive technology in the new millennium: part II. Urology 2004; 63: 217-224.

6 Knoppers BM, Isasi RM: Regulatory approaches to reproductive genetic testing. Hum Reprod 2004; 19: 2695-2701.

7 Sermon K, Moutou C, Harper J et al: ESHRE PGD Data Collection IV. Hum Reprod 2005; 20: 19-34, (Epub 2004 Nov 18).

8 Wilton L: Preimplantation genetic diagnosis for aneuploidy screening in early human embryos: a review. Prenat Diagn 2002; 22: $312-318$

9 Staessen C, Platteau P, Van Assche E et al: Comparison of blastocyst transfer with or without preimplantation genetic diagnosis for aneuploidy screening in couples with advanced maternal age: a prospective randomizes controlled trial. Hum Reprod Adv Access 2004.
10 Ingerslev HJ, Poulsen PB, Højgaard A et al: Proeimplantationsdiagnostik - En medicinsk teknologivurdering. Copenhagen, 2002, http://www.sst.dk/Applikationer/cemtv/publikationer/docs/ Praeimplantation/html/praeimplant.pdf (For English summary: http://www.sst.dk/Applikationer/cemtv/publikationer/docs/ Praeimplantation/html/sum.htm.

11 Adashi EY, Barri N, Berkowitz R et al: Infertility therapyassociated multiple pregnancies (births): an ongoing epidemic, report of the meeting. RBM (online) 2003; 7: 515-542.

12 Bonduelle M, Wennerholm U-B, Loft A et al: A multi-centre cohort study of the physical health of 5-year-old children conceived after intracytoplasmic sperm injenction, in vitro fertilization and natural conception. Hum Reprod 2005; 20: 413-419.

13 Hansen M, Bower C, Milne E, de Klerk N, Kurinczuk J: Assisted reproductive technologies and the risk of birth defects - a systematic review. Hum Reprod 2005; 20: 328-338.

14 Klemetti R, Gissler M, Sevón T, Koivurova S, Ritvanen A, Hemminki E: Children born after assisted fertilization have an increased rate of major congenital anomalies. Fertil Steril 2005; 84: 1300-1307.

15 Kurinczuk J, Hansen M, Bower C: The current birth defects in children born after assisted reproductive technologies. Curr Opin Obstet Gynecol 2004; 16: 201-209.

16 Ludwig M, Katalinic A, Gross S, Sutcliffe A, Varon R, Horsthemke $B$ : Increased prevalence of imprinting defects in patients with Angelman syndrome born to subfertile couples. I Med Genet 2005; 42: 289-291.

17 Min JK, Breheny SA, MacLachlan V, Healy DL: What is the most relevant standard of success in assisted reproduction? The singleton, term gestation, live birth rate per cycle initiatd: the BESST endpoint for assisted reproduction. Hum Reprod 2004; 19: $3-7$.

18 WHO: Current Practices and controversies in assisted reproduction. Report of a WHO Meeting 2001. WHO: Geneva, 2002.

19 Parens E, Knowles LP: The Hastings Center: reprogenetics and public policy, reflections and recommendations, July-August 2003.

20 ESHRE Capri Workshop Group. : Hum Reprod 2000; 15: 1856 1864.

21 ESHRE Capri Workshop Group: Optimal use of infertility diagnostic tests and treatments. Hum Reprod 2000; 15: 723-732.

22 Aittomaki K, Wennerholm UB, Bergh C, Selbing A, Hazekamp J, Nygren KG: Safety issues in assisted reproduction technology: should ICSI patients have genetic testing before treatment? A practical proposition to help patient information. Hum Reprod 2004; 19: 472-476.

23 Geraedts JP, Harper J, Braude P et al: Preimplantation genetic diagnosis (PGD), a collaborative activity of clinical genetic departments and IVF centres. Prenat Diag 2001; 21: 1086-1092.

24 Sermon K, Van Steirteghem A, Liebaers I: Preimplantation genetic diagnosis. Lancet 2004; 363: 1633-1641.

25 IFFS - International Federation of Fertility Societies: IFFS Surveillance 04. Fertil Steril 2004; 81 (Suppl 4) http://www.asrm. org/Professionals/Fertility\&Sterility/iffs_surveillance_2004.pdf.

26 De Wert G: Ethics of assisted reproduction; in Fauser B (ed): Reproductive Medicine. New York: Parthenon Publishing, 2002, pp 645-665.

27 De Wert G: Ethical Aspects of prenatal testing and preimplantation genetic diagnosis for late-onset neurogenetic disease: the case of Huntington's disease; in Evers-Kiebooms G, Zoeteweij m, Harper P (eds): Prenatal Testing for Late-onset Neurogenetic Diseases. BIOS Scientific Publishers Ltd: Oxford, 2002, pp 129-157.

28 Conti A, Delbon P, Sirignano A: Informed consent when taking genetic decisions. Med Law 2004; 23: 337-353.

29 WHO: Review of Ethical Issues in Medical Genetics. WHO: Geneva, 2003.

30 Houmard BS, Seifer DB: Infertility treatment and informed consent: current practises by reproductive endocrinologists. Obstet Gynecol 1999; 93: 252-257. 
31 Ayhan A, Salman MC, Celik H, Dursun P, Ozyuncyu O, Gultekin M: Association between fertility drugs and gyneologic cancers, breast cancer, and childhood cancers. Acta ObstetGynecol Scand 2004; 83: 1104-1111.

32 Schieve L, Rasmussen S, Reefhuis J: Risk of birth defects among children conceived with assisted reproductive technology: providing an epidemiologic context to the data. Fertil Steril 2005; 84: 1320-1324.

33 Sutcliffe AG: Congenital anomalies and assisted reproductive technology: more of the same. Fertil Steril 2005; 84: 1316-1317.

34 Olivennes F: Do children born after assisted reproductive technology have a higher incidence of birth defects? Fertil Steril 2005; 84: 1325-1326.

35 Michie S, McDonald V, Marteau TM: Genetic counselling: information given, recall and satisfaction. Patient Educ Counsel 1997; 32: 101-106.

36 Foresta C, Ferlin A, Gianaroli L, Dallapiccola B: Guidelines for the appropriate use of genetic tests in infertile couples. Eur J Hum Genet 2002; 10: 303-312.

37 Vogt P: Genomic heterogeneity and instability of the AZF locus on the human Y chromosome. Mol Cell Endocrinol 2004; 224: $1-9$.

38 Rockett JC: Meeting report: genomic and proteomic techniques applied to reproductive biology. Genome Biol 2001; 2: 4020.14020.

39 Schlegel PM: Causes of azoospermia and their management. Reprod Fertil Dev 2004; 16: 561-572.

40 Foresta C, Grolla A, Bartoloni L, Bettella A, Ferlin A: Genetic abnormalities among severely oligospermic men who are candidates for intracytoplasmic sperm injection. J Clin Endocrinol Metab 2005; 90: 152-156.

41 Bourrouillou G, Bujan L, Calvas P, Colombies P, Mansat A, Pontonnier F: Role and contribution of karyotyping in male infertility. Prog Urol 1992; 2: 189-895.

42 Seifer I, Amat S, Delgado-Viscogliosi P, Boucher D, Bignon YJ: Screening for microdeletions on the long arm of chromosome $Y$ in 53 infertile men. Int J Androl 1999; 22: 148-154.

43 Foresta C, Moro E, Ferlin A: Prognostic value of Y deletion analysis. The role of current methods. Hum Reprod 2001; 16: $1543-1547$.

44 Tiepolo L, Zuffardi O: Localization of factors controlling spermatogenesis in the nonfluorescent portion of the human Y chromosome long arm. Hum Genet 1976; 34: 119-124.

45 Vogt P: Basic science meets the clinic, introduction in Symposium of Genetic aspects of male (in)fertility. RBM (online) 2005; 10: $11-13$.

46 Johnson MD: Genetic risks of intracytoplasmic sperm injection in the treatment of male infertility: recommendations for genetic counseling and screening. Fertil Steril 1998; 70: $397-411$.

47 Bernardini L, Borini A, Preti S et al: Study of aneuploidy in normal and abnormal germ cells from semen of fertile and infertile men. Hum Reprod 1998; 13: 3406-3413.

48 Pang MG, Hoegerman SF, Cuticchia AJ et al: Detection of aneuploidy for chromosomes 4, 6, 7, 8, 9, 10, 11, 12, 13, 17, 18, $21, X$ and $Y$ by fluorescence in-situ hybridization in spermatozoa from nine patients with oligoasthenoteratozoospermia undergoing intracytoplasmic sperm injection. Hum Reprod 1999; 14: 1266-1273.

49 Burrello N, Vicari E, Shin P et al: Lower sperm aneuploidy frequency is associated with high pregnancy rates in ICSI programmes. Hum Reprod 2003; 18: 1371-1376.

50 Ferlin A, Garolla A, Foresta C: Chromosome abnormalities in sperm of individuals with constitutional sex chromosomal abnormalities. Cytogenet Gen Res 2005; 111 (3-4): 310-316.

51 Poulakis V, Witzsch U, Diehl W, de Vries R, Becht E, Trotnow S: Birth of two infants with normal karyotype after intracytoplasmic injection of sperm obtained by testicular extraction from two men with nonmosaic Klinefelter's syndrome. Fertil Steril 2001; 76: 1060-1062.
52 Rubio C, Gil-Salom M, Simon C et al: Incidence of sperm chromosomal abnormalities in a risk population: relationship with sperm quality and ICSI outcome. Hum Reprod 2001; 16: 2084-2092.

53 Arán B, Blanco J, Vidal $\mathrm{F}$ et al: Screening for abnormalities of chromosomes $\mathrm{X}$, Y and 18 and for diploidy in spermatozoa from infertile men included in an IVF-ICSI program. Fertil Steril 1999; 72: $696-701$.

54 Egozcue J, Blanco J, Anton E, Egozcue S, Sarrate Z, Vidal F: Genetic analysis of sperm and implications of severe male infertility. A review. Placenta 2003; 24: S62-S65.

55 Silber S, Escudero T, Lenahan K, Abdelhadi I, Kilani Z, Munne S: Chromosomal abnormalities in embryos derived from testicular sperm extraction. Fertil Steril 2003; 79: 30-38.

56 Claustres M: Molecular pathology of the CFTR locus in male. Symposium: genetic aspects of male (in)fertility. RBM (online) 2005; 10: 14-41.

57 Kaplan E, Shwachman H, Perlmutter AD, Rule A, Khaw KT, Holsclaw DS: Reproductive failure in males with cystic fibrosis. N Engl J Med 1968; 279: 65-69.

58 Rigot JM, Lafitte JJ, Dumur V et al: Cystic fibrosis and congenital absence of the vas deferens. N Engl J Med 1991; 325: 64-65.

59 Anguiano A, Oates RD, Amos JA et al: Congenital bilateral absence of the vas deferens. A primarily genital form of cystic fibrosis. JAMA 1992; 267: 1794-1797.

60 Weiske WH, Salzler N, Schroeder-Printzen I, Weidner W: Clinical findings in congenital absence of the vasa deferentia. Andrologia 2000; 32: 13-18.

61 McCallum T, Milunsky J, Munarriz R, Carson R, Sadeghi-Nejad $\mathrm{H}$, Oates R: Unilateral renal agenesis associated with congenital bilateral absence of the vas deferens: phenotypic findings and genetic considerations. Hum Reprod 2001; 16: 282-288.

62 Källén B, Finnström O, Nygren KG et al: In vitro fertilisation (IVF) in Sweden: risk for congenital malformations after different IVF methods. Birth Defects Res A Clin Mol Teratol 2005; 73 (3): 162-169.

63 Hultén MA, Barlow AL, Tease C: Meiotic studies in humans; in Rooney DE (ed): Human Cytogenetics: Constitutional Analysis, Third Edition. Oxford: Oxford University Press, 2001.

64 Egozcue J, Sarrate Z, Codina-Pascual M et al: Meiotic abnormalities in infertile males; in Martin RH (ed): Cytogenetic and Genome Research 2005; 111 (3-4): 337-342.

65 Arán B, Veiga A, Vidal F et al: Preimplantattion genetic diagnosis in patients with male meiotic abnormalities. Reprod Biomed (online) 2004; 8: 470-476.

66 Jequier AM: Clinical andrology - still a major problem in the treatment of infertility. Hum Reprod 2004; 19: 1245-1249.

67 Cuppens H, Cassiman JJ: CFTR mutations and polymorphisms in male infertility. Int J Androl 2004; 27: 251-256.

68 Bernardini LM, Costa M, Bottazzi C et al: Sperm aneuploidy and recurrent pregnancy loss. Reprod Reprod Biomed (online) 2004.

69 Palermo GD, Colombero LT, Hariprashad JJ, Schlegel PN, Rosenwaks Z: Chromosome analysis of epidymal and testicular sperm in azoospermic patients undergoing ICSI. Hum Reprod 2002; 17: 570-575.

70 Guidelines for gamete and embryo donation: Fertil Steril 2002; 77 (Suppl 5): S1-S16.

71 Human Fertilisation and Embryology Authority (HFEA), www.hfea.gov.uk. Code of Practise, 6th edn. 2004.

72 Thurin A, Hausken J, Hillensjo T et al: Elective single-embryo transfer versus double-embryo transfer in in vitro fertilization. $N$ Engl J Med 2004; 351: 2440-2442.

73 Martikainen H, Tiitinen A, Tomas C et al: Study Group: one versus two embryo transfer after IVF and ICSI: a randomized study. Hum Reprod 2001; 16: 1900-1903.

74 Bergh C, Wennerholm UB: What is the most relevant standard of success in assisted reproduction? Singleton live births should also include preterm births. Hum Reprod 2004; 19: 1943-1945.

75 De-Die Smulders C, Lulofs R, Muntjewerff N, Geraedts J, Land J: Profiles and motives of couples referred for preimplantation genetic diagnosis (PGD). Hum Reprod 2005, (submitted). 
76 Shenfield F, Pennings G, Devroye P, Sureau C, Tarlatzis B, Cohen J: ESHRE Taskforce 5: preimplantation genetic diagnosis. Hum Reprod 2003; 18: 649-651.

77 Thornhill AR, De Die-Smulders CE, Geraedts JP et al: ESHRE PGD Consortium 'Best practice guidelines for clinical preimplantation genetic diagnosis (PGD) and preimplantation genetic screening (PGS)'. Hum Reprod 2005; 20: 35-48, (Epub 2004 Nov 11).

78 De Boer KA, Catt JW, Jansen RPS, Leigh D, McArthur S: Moving to blastocyst biopsy for preimplantation genetic diagnosis and single embryo transfer at Sydney IVF. Fertil Steril 2004; 82: 295-298.

79 Ethikrat. (German national ethics council): Genetic diagnosis before and during pregnancy 2003.

80 PGDIS - Preimplantation Genetic Diagnosis International Society: PGDIS - Preimplantation Genetic Diagnosis International Society: guidelines for good practice in PGD. Reprod Biomedicine (online) 2004; 9: 430-434.

81 Ström CM, Levin R, Ström S, Masciangelo C, Kuliev A, Verlinsky Y: Neonatal outcome of preimplantation genetic diagnosis by polar body removal: the first 109 infants. Pediatrics 2000; 106: $650-653$.

82 Gianaroli L, Magli MC, Ferraretti AP: Preimplantation genetic diagnosis in Current Practices and controversies in assisted reproduction. Report of a WHO Meeting 2001. Geneva: WHO, 2002.

83 Ethikrat. (German national ethics council) www.ethikrat.org. Polar Body Diagnosis 2004.

84 Rechitsky S, Ström S, Verlinsky O et al: Accuracy of preimplantation diagnosis of single-gene disorders by polar body analysis of oocytes. J Assist Reprod Genet 1999; 16: 192-198.

85 Antczak M, VanBlerkom J: Temporal and spatial aspects of fragmentation in early human embryos: possible effects on developmental competence and association with the differential elimination of regulatory proteins from polarized domains. Hum Reprod 1999; 14: 429-447.

86 Ziebe S, Lundin K, Loft A, et al: CEMAS II and Study Group: FISH analysis for chromosomes 13, 16, 18, 21, 22, $\mathrm{X}$ and $\mathrm{Y}$ in all blastomeres of IVF pre-embryos from 144 randomly selected donated human oocytes and impact on pre-embryo morphology. Hum Reprod 2003; 18: 2575-2581.

87 Milki AA, Jun SH, Hinckley MD, Behr B, Giudice LC, Westphal LM: Incidence of monozygotic twinning with blastocyst transfer compared to cleavage-stage transfer. Fertil Steril 2003; 79: 503-506

88 Scriven PN, Flinter FA, Braude PR, Ogilvie CM: Robertsonian translocations - reproductive risks and indications for preimplantation genetic diagnosis. Hum Reprod 2001; 16: 2267-2273.

89 Evers-Kiebooms G, Zoeteweij M, Harper P (eds): Prenatal Testing for Late-onset Neurogenetic Diseases. BIOS Scientific Publishers Ltd: Oxford, 2002; 220pp.

90 De Die C, De Heurckmans N: Case histories about preimplantation genetic diagnosis; in Evers-Kiebooms G, Zoeteweij M, Harper P (eds): Prenatal Testing for Late-onset Neurogenetic Diseases. BIOS Scientific Publishers Ltd: Oxford, 2002, pp 119-127.

91 Geraedts J, Liebaers I: Preimplantation genetic diagnosis for Huntington's disease; in Evers-Kiebooms G, Zoeteweij MW, Harper PS (eds): Prenatal testing for Late-onset Neurogenetic Diseases. BIOS Scientific Publishers Ltd: Oxford, 2002.

92 Sermon K, De Rijcke M, Lissens W et al: Preimplantation genetic diagnosis for Huntington's disease with exclusion testing. Eur J Hum Genet 2002; 10: 591-598.

93 Moutou C, Gardes N, Viville S: New tools for preimplantation genetic diagnosis of Huntington's disease and their clinical applications. Eur J Hum Genet 2004; 12: 1007-1014.

94 Stern HJ, Harton GL, Sisson ME et al: Non-disclosing preimplantation genetic diagnosis for Huntington's disease. Prenat Diagn 2002; 22: 503-507.

95 Braude PR, de Wert GMWR, Evers-Kiebooms G, Pettigrew RA, Geraedts JP: Non-disclosure preimplantation genetic diagnosis for Huntington's disease: practical and ethical dilemma. Prenat Diagn 1998; 18: 1422-1426.
96 Clarke AJ in Harper PS, Clarke AJ (eds): Genetics, Society and Clinical Practise: The Process of Genetic Counselling: Beyond NondirectIveness. BIOS Scientific Publishers Ltd: Oxford, 1997.

97 Munne S: Preimplantation genetic diagnosis of numerical and structural chromosome abnormalities. Reprod Biomed (online) 2002; 4: 183-196.

98 UNESCO: International Bioethics Committee (IBC): Report on PGD and Germ-Line Intervention, 2003.

99 Wilton L: Preimplantation genetic diagnosis and chromosome analysis of blastomeres using comparative genome hybridization. Hum Reprod Update 2004.

100 Flinter FA: Preimplantation genetic diagnosis [editorial]. BM) 2001; 322: 1008-1009.

101 Viville S, Pergament D: Results of a survey of the legal status and attitudes towards preimplantation genetic diagnosis conducted in 13 different countries. Prenat Diagn 1998; 1374-1380.

102 Briggs DA, Power NJ, Lamb V, Rutherford JA, Gosden RG: Amplification of DNA sequences in polar bodies from human oocytes for diagnosis of mitochondrial disease. Lancet 2000; 355: $1520-1521$.

103 Kuliev A, Verlinsky Y: Preimplantation genetic diagnosis: a realistic option for assisted reproduction and genetic practice. Obstet Gynecol 2005; 17: 179-183.

104 Goldman JM, Schmitz N, Niethammer D, Gratwohl A: Allogeneic and autologous transplantation for haematological diseases, solid tumours and immune disorders: current practice in Europe in 1998. Bone Marrow Transplant 1998; 21: 1-7.

105 Pennings G, Schots R, Liebaers I: Ethical considerations on preimplantation genetic diagnosis for HLA typing to match a future child as a donor of hematopoietic stem cells to a sibling. Hum Reprod 2002; 17: 534-538.

106 Rechitsky S, Kuliev A, Tur-Kaspa I, Morris R, Verlinsky Y: Preimplantation genetic diagnosis with HLA matching. Reprod Biomed (online) 2004; 9: 210-221.

107 Fiorentino F, Biricik A, Karadayi $\mathrm{H}$ et al: Development and clinical application of a strategy for preimplantation genetic diagnosis of single gene disorders combined with HLA matching. Mol Hum Reprod 2004; 10: 445-460.

108 Van de Velde H, Georgiou I, De Rycke M et al: Novel universal approach for preimplantation genetic diagnosis of beta-thalassaemia in combination with HLA matching of embryos. Hum Reprod 2004; 19: 700-708.

109 Kahraman S, Karlikaya G, Sertyel S, Karadayi H, Findikli N, Oncu $\mathrm{N}$ : Clinical aspects of preimplantation genetic diagnosis for single gene disorders combined with HLA typing. Reprod Biomed (online) 2004; 9: 529-532.

110 Kearney W, Caplan AL: Parity for donation; in Blank RH, Bonnicksen AL (eds): Emerging Issues in Biomedicine Policy. New York: Columbia University Press, 1992, vol 1, pp 263-285.

111 Verlinsky Y, Cohen J, Munne S et al: Reproductive Genetics Institute. Over a decade of experience with preimplantation genetic diagnosis: a multicenter report. Fertil Steril 2004; 82 : 292-294.

112 De Die-Smulders C, Land JA, Dreesen JC, Coonen E, Evers JL, Geraedts JP: Results from 10 years of preimplantation-genetic diagnostics in the Netherlands [in Dutch]. Ned Tijdschr v Geneesk 2004; 148: $2491-2496$.

113 Marjoribanks J, Farquhar C, Marshall C, Cook Rob/the Cochrane Menstrual Disorders and Subfertility Group (CMDSG) for the New Zealand Guidelines Group (NZGG): Systematic review of the quantifiable harms and benefits of preimplantation genetic diagnosis (PGD): Advice for the National Ethics Committee on Assisted Human Reproduction and the Ministry of Health, September 2004, New Zealand.

114 Lavery SA, Aurell R, Turner C et al: Preimplantation genetic diagnosis: patients' experiences and attitudes. Hum Reprod 2002; 17: $2464-2467$

115 Katz MG, Fitzgerald L, Bankier A, Savulescu J, Cram DS: Issues and concerns of couples presenting for preimplantation genetic diagnosis (PGD). Prenat Diagn 2002; 22: 1117-1122. 
116 Human Fertilisation and Embryology Authority (HFEA): Sex selection: options for regulation, 2003.

117 Harris J: Sex selection and regulated hatred. J Med Ethics 2005; 31: 291-294.

118 Harris J: No sex selection please, we're British. J Med Ethics 2005; 31: $286-288$.

119 Baldwin T: Reproductive liberty and elitist contempt: reply to John Harris. J Med Ethics 2005; 31: 288-290.

120 Dahl E: Boy or Girl: Should Parents be Allowed to Choose the Sex of Their Children? Cardiff Centre for Ethics Law \& Society. February 2005, http://www.ccels.cardiff.ac.uk/literature/ publications/2005/dahlpaper.html.

121 Ray PF, Munnich A, Nisand I, Frydman R, Vekemans M, Viville S: the French GET -DPI: the place of 'social sexing' in medicine and science. Hum Reprod 2002; 17: 248-249.

122 The Council of Europe: Convention on Human Rights and Biomedicine 1997.

123 Shakespeare T: Ethics watch, sex selection. Nat Genet Rev 2005; 6: 666.

124 Human Genetics Commission (HGC), www.hgc.gov.uk. Choosing the future: genetics and reproductive decision making; a discussion paper June 2004, http://www.hgc.gov.uk/ choosingthefuture/ChooseFuturefull.pdf.

125 Krones T, Schluter E, Manolopoulos K et al: Public, expert and patients, opinions towards preimplantation genetic diagnosis (PGD) in Germany. RBM Online Public, vol 10, no. 1, January 2005.

126 Pennings G: Legal harmonisation and reproductive tourism in Europe. Hum Reprod 2004; 19: 2689-2694.

127 King DS: Preimplantation genetic diagnosis and the new eugenics. J Med Ethics 1999; 25: 176-182.

128 Human Fertilisation and Embryology Authority (HFEA): Seed review, 2004.

129 Vance A, Zouves C: The importance of family risk assessment in infertility setting. Fertil Steril 2005; 84: S125.

130 Janssens PMW: No reason for a reduction in the number of offspring per sperm donor because of possible transmission of autosomal diseases. Hum Reprod 2003; 18: 669-671.

131 Jacquard A, Schoëvaërt D: Insémination et consanguinité. Ann Génét 1976; 19: 229-231.

132 Eydoux P, Thepot F, Fellmann F, et al: the Commission de Génétique de la Fédération Francaise des CECOS: How can the genetic risks of embryo donation be minimized? Proposed guidelines of the French Federation of CECOS (Centre dÉtude et de Concervation des Oeufs et du Sperme). Hum Reprod 2004; 19 : $1685-1688$

133 Shiloh S, Saxe L: Perception of risk in genetic counselling. Psychol Health 1989; 3: 45-61.

134 Godard B, Kääriäinen H, Kristoffersson U, Tranebjaerg L, Coviello D, Aymé S: Provision of genetic services in Europe: current practises and issues. Eur J Hum Genet 2003; 11 (Suppl 2): S13-S48.

135 IPTS - Institute of Prospective Technological Studies: Towards quality assurance and harmonisation of genetic testing services in the EU, 2003.

136 Emery M, Béran M-D, Darwiche $\mathrm{J}$ et al: Results from a prospective, randomized, controlled study evaluating the acceptability and effects of routine pre-IVF counselling. Hum Reprod 2003; 18: 2647-2653.

137 Van Zuuren FJ, van Schie ECM, van Baaren NK: Uncertainty in the information provided during genetic counseling. Patient Educ Counsel 1997; 32: 129-139.

138 Kessler S: Psychological aspects of genetic counselling. XI. Nondirectiveness revisited. Am J Med Genet 1997; 72: 164-171.

139 Rogers CR: Counseling and Psychotherapy: Newer Concepts in Practice. Boston: Houghton Mifflin, 1942.

140 Rogers CR: Client-Centered Therapy: Its Current Practice, Implications and Theory. Boston: Houghton Mifflin, 1951.

141 Welkenhuysen M, Evers-Kiebooms G, d'Ydewalle G: The language of uncertainty in genetic risk communication: framing and verbal versus numerical information. Patient Educ Counsel 2001; 43: 179-187.

142 Elwyn G, Gray J, Clarke A: Shared decision making and nondirectiveness in genetic counselling. J Med Genet (online) 2000; 37: $135-138$.

143 Edwards A, Elwyn G, Covey J, Matthews E, Pill R: Presenting risk information - a review of the effects of 'framing' and other manipulations on patient outcomes. J Health Commun 2001; 6 : 61-82.

144 Gekas J, Gondry J, Mazur S, Cesbron P, Thepot F: Informed consent to serum screening for Down syndrome: are women given adequate information. Prenat Diagn 1999; 19: 1-7.

145 Marteau TM, Nippert I, Hall S et al: Outcomes of pregnancies diagnosed with Klinefelter syndrome: the possible influence of health professionals. Prenat Diagn 2002; 22: 562-566.

146 Lambert RD: Safety issues in assisted reproduction technology: aetiology of health problems in singleton ART babies. Hum Reprod 2003; 10: 1987-1991.

147 Wennerholm UB: Infertility therapy-associated multiple pregnancies (births): an ongoing epidemic, report of the meeting. RBM (online) 2003; 7: 515-542.

148 Hazekamp J, Bergh C, Wennerholm UB, Hovatta O, Karlström PO, Selbing A: Debate: avoiding multiple pregnancies in ART: consideration of new strategies. Hum Reprod 2000; 15: $1217-1219$

149 Shenfield F: Infertility therapy-associated multiple pregnancies (births): an ongoing epidemic, report of the meeting. RBM (online) 2003; 7: 515-542.

150 Vilska S, Tiitinen A, Hyden-Granskog C, Hovatta O: Elective transfer of one embryo results in an acceptable pregnancy rate and eliminates the risk of multiple birth. Hum Reprod 1999; 14: 2392-2395.

151 Gerris Jan MR: Review: single embryo transfer and IVF/ICSI outcome: a balanced appraisal. Hum Reprod Update 2004, (advance access published online on October 28)

152 Salumets A, Hyden-Granskog C, Makinen S, Suikkari AM, Tiitinen A, Tuuri T: Early cleavage predicts the viability of human embryos in elective single embryo transfer procedures. Hum Reprod 2003; 18: 821-825.

153 Slotnick R et al: Monoamniotic twinning and zona manipulation: a survey of US IVF centers correlating zona manipulation procedures and high-risk twinning frequency. J Assist Reprod and Genet 1996; 13: 381

154 Schieve LA et al: Does assisted hatching pose a risk for monozygotic twinning in pregnancies conceived through in vitro fertilisation. Fertil Steril 2000; 74: 288-294.

155 Braude P, Rowell P: Assisted conception III - problems with assisted conception. BMJ 2003; 327: 920-923.

156 Hreinsson J, Rosenlund B, Fridén B et al: Recombinant LH is equally effective as recombinant hCG in promoting oocyte maturation in a clinical in-vitro maturation programme: a randomized study. Hum Reprod 2003; 18: 2131-2136.

157 Gauthier E, Paoletti X, Clavel-Chapelon F, the E3N group: Breast cancer risk associated with being treated for infertility: results from the French E3N cohort study. Hum Reprod 2004; 19: 22162221.

158 Brinton LA, Lamb EJ, Moghissi KS et al: Ovarian cancer risk after the use of ovulation-stimulating drugs. Obstet Gynecol 2004; 103: $1194-1203$.

159 Hansen M, Kurinczuk JJ, Bower C, Webb S: The risk of major birth defects after intracytoplasmic sperm injection and in vitro fertilization. N Engl J Med 2002; 346: $725-730$.

160 Schieve L, Meikle S, Ferre Cym: Low and very low birth weight in infants conceived with use of assisted reproductive technology. N Engl J Med 2002; 346: 731-737.

161 Pinborg A, Loft A, Nyboe Andersen A: Neonatal outcome in a Danish national cohort of 8602 children born after in vitro fertilization or intracytoplasmic sperm injection: the role of twin pregnancy. Acta Obstet Gynecol Scand 2004; 83: $1071-1078$ 
162 Wennerholm UB: Cryopreservation of embryos and oocytes: obstetric outcome and health in children. Hum Reprod 2000; 15 (Suppl 5): 18-25.

163 Koivurova S, Hartikainen A-L, Gissler M, Hemminki E, Sovio U, Järvelin M-R: Neonatal outcome and congenital malformations in children born after in-vitro fertilization. Hum Reprod 2002; 17: 1391-1398.

164 Hampton T: Panel reviews health effects data for assisted reproductive technologies. JAMA 2004; 292: 2961-2962.

165 Lidegaard O, Pinborg A, Andersen AN: Imprinting diseases and IVF: Danish National IVF cohort study. Hum Reprod 2005; 20: 950-954.

166 Moll AC, Ihhof SM, Cruysberg JRM, Schouten-van Meeteren AYN, Boers M, Van Leeuwen FE: Incidence of retinoblastoma in children born after in-vitro fertilisation. Lancet 2003; 361: 309-311.

167 De Rycke M, Liebaers I, Van Steirteghem A: Epigenetic risks related to assisted reproductive technologies. Risk analysis and epigenetic inheritance. Hum Reprod 2002; 17: 2487-2494.

168 Shi W, Haaf T: Aberrant methylation patterns at the two-cell stage as an indicator of early developmental failure. Mol Reprod Dev 2002; 63: 329-334.

169 Cox GF, Bürger J, Lip V, Mau UA, Wu BL, Horsthemke B: Intracytoplasmic sperm injection may increase the risk of imprinting defects. Am J Hum Genet 2002; 71: 162-164.

170 Orstavik KH, Eiklid K, Van der Hagen CB et al: Another case of imprinting in a girl with Angelman syndrome who was conceived by ICSI. Am J Hum Genet 2003; 72: 218-219.

171 De Baun MR, Niemitz EL, Feinburg AP: Association of in vitro fertilisation with Beckwith-Wiedemann syndrome and epigenetic alterations of LIT1 anh H19. Am J Hum Genet 2003; 72: $156-160$.

172 Maher ER, Brueton LA, Bowdin SC et al: Beckwith-Wiedemann syndrome and assisted reproduction technology (ART). J Med Genet 2003; 40: 62-64.

173 Gicquel C, Gaston V, Mandelbaum J, Siffroi JP, Flahault A, Le Bouc Y: In vitro fertilisation may increase the risk of BeckwithWiedemann syndrome related to abnormal imprinting of the KCN1OT gene. Am J Hum Genet 2003; 72: 1338-1341, (PMID: 12772698 (PubMed)).

174 Halliday J, Oke K, Breheny S, Algar E, Amor D: BeckwithWiedemann syndrome and IVF: a case-control study. Am J Hum Genet 2004; 75: 526-528, (PMID: 15284956 (PubMed)).

175 Marques CJ, Carvalho F, Souza M, Barros A: Genomic imprinting in disruptive spermatogenesis. Lancet 2004; 363: 1700-1702.

176 Gosden R, Trasler J, Lucifero D, Faddy M: Rare congenital disorders, imprinted genes, and assisted reproductive technology. Lancet 2003; 361: 1975-1977.

177 Winston RM, Hardy K: Are we ignoring potential dangers of in vitro fertilization and related treatments? Nat Cell Biol Nat Med 2002; 8: S14-S18.

178 Lucifero D, Chaillet J, Richard M, Trasler J: Potential significance of genomic imprinting defects for reproduction and assisted reproductive technology. Hum Reprod Update 2004; 10: 3-18.

179 Dumoulin JC, Derhaag JG, Bras M et al: Growth rate of human preimplantation embryos is sex dependent after ICSI but not after IVF. Hum Reprod 2005; 20: 484-491.

180 Golombok S: Parenting and the psychological development of the child in ART families; in Vayena E, Rowe PJ, Griffin PD (eds): Current Practices and Controversies in Assisted Reproduction, Report of a WHO Meeting 2001. Geneva: WHO, 2002, pp 287-302.

181 Braude P, Rowell P: Assisted conception. II - in vitro fertilisation and intracytoplasmic sperm injection. BMJ 2003; 327: 852-855.

182 Bondulle M, Bergh C, Niklasson A et al: Medical follow-up study of 5-year-old ICSI children. RBM (online) 2004; 9: 91-101.

183 CCNE - Comité consultatif national d'éthique pout les sciences de la vie et de la santé (CCNE): Ethical issues raised by the development of ICSI. Opinion no: 75, 12 December 2002.

184 CCNE - Comité consultatif national d'éthique pour les sciences de la vie et de la santé (CCNE): Reflections concerning an extension of Preimplantation Genetic Diagnosis. No. 72, 2002.

185 Devroey P, Van Steirteghem A: A review of ten years experience of ICSI. Hum Reprod Update 2004; 10: 19-28.

186 Ponjaert-Kristoffersen I, Tjus T, Nekkebroeck J et al: Psychological follow-up study of 5-year-old ICSI children. Hum Reprod 2004; 19: 2791-2797.

187 Van Steirteghem A: ICSI: micromanipulation in assisted fertilization in 'Current Practices and controversies in assisted reproduction'. Report of a WHO Meeting. Geneva: WHO, 2002.

188 Vandervorst M, Staessen C, Sermon K et al: The Brussels' experience of more than 5 years of clinical preimplantation genetic diagnosis. Hum Reprod 2000; 6: 364-373.

189 Iwarsson E, Lundqvist $\mathrm{M}$, Inzunza $\mathrm{J}$ et al: A high degree of aneuploidy in frozen-thawed human preimplantation embryos. Hum Genet 1999; 104: 376-382.

190 Boiso I, Marti M, Santalo J, Ponsa M, Barri PN, Veiga A: A confocal microscopy analysis of the spindle and chromosome configurations of human oocytes cryopreserved at the germinal vesicle and metaphase II stage. Hum Reprod 2002; 17: $1885-1891$

191 Chen SU, Lien YR, Chao KH, Ho HN, Yang YS, Lee TY: Effects of cryopreservation on meiotic spindles of oocytes and its dynamics after thawing: clinical implications in oocyte freezing - a review article. Mol Cell Endocrinol 2003; 202: 101-107.

192 Chian RC, Buckett WM, Abdul Jalil AK et al: Natural-cycle in vitro fertilization combined with in vitro maturation of immature oocytes is a potential approach in infertility treatment. Fertil Steril 2004; 82: 1168-1675.

193 Cohen J, Scott R, Schimmel T, Levron J, Willadsen S: Birth of infant after transfer of anucleate donor oocyte cytoplasm into recipient eggs. Lancet 1997; 350: 186-187.

194 Barritt J, Willadsen S, Brenner C, Cohen J: Epigenetic and experimental modifications in early mammalian development: Part II. Cytoplasmic Transfer in Assisted Reproduction. Hum Reprod Update 2001; 7: 428-435

195 Schatten G: Safeguarding ART. Nat Cell Biol Nat Med 2002; S19-S22.

196 Bonduelle M, Liebaers I, Deketelaere V et al: Neonatal data on a cohort of 2889 infants born after ISCI (1991-1999) and of 2995 infants born after IVF (1983-1999). Hum Reprod 2002; 17: 671.

197 Bonduelle M, Bergh C, Niklasson A et al: Medical follow-up study of 5-year-old ICSI children. RBM Online 2004; 9: 91-101.

198 Bonduelle M, Ponjaert I, Steirteghem AV, Derde MP, Devroey P, Liebaers I: Developmental outcome at 2 years of age for children born after ICSI compared with children born after IVF. Hum Reprod 2003; 18: 342-350.

199 European Molecular Genetics Quality Network - EMQN, www.emqn.org.

200 Bateman S: When reproductive freedom encounters medical responsibility: changing conceptions of reproductive choice, in Current Practices and controversies in assisted reproduction. Report of a WHO Meeting 2001. WHO: Geneva, 2002.

201 Nys H, Casabona CM, Desmet C: Legal aspects of prenatal testing for late-onset neurological diseases; in Evers-Kiebooms G, Zoeteweij MW, Harper PS (eds): Prenatal Testing for Late-Onset Neurogenetic Diseases. BIOS Scientific Publishers Ltd: Oxford, 2002.

202 Mason JK, McCall Smith RA, Laurie GT: Law and Medical Ethics. Butterworths, 2002.

203 PCBE: The President's Council on Bioethics: Reproduction and responsIbility: The Regulation of New Biotechnologies. Washington, 2004.

204 Paoloni-Giacobino A, Chaillet RJ: Genomic imprinting and assisted reproduction. Reprod Health 2004; 1: 6.

205 Hardy K, Wright C, Rice S et al: Future developments in assisted reproduction in humans. Reproduction 2002; 123: $171-183$.

206 Kanavakis E, Traeger-Synodis J: Preimplantation genetic diagnosis in clinical practise. J Med Genet 2002; 39: 6-11. 
207 Ola B et al: Should ICSI be a treatment of choice for all cases of in-vitro conception? Considerations of fertilization and embryo development, cost effectineness and safety. Hum Reprod 2001; 16: $2485-2490$.

208 Montfoort APA, Dumoulin JCM, Land JA, Coonen E, Derhaag JG, Evers JLH: Elective single embryo transfer (eSET) policy in the first three IVF/ICSI treatment cycles. Hum Reprod 2005; 20: $433-436$.

209 De Wert G, Mummery C: Human embryonic stem cells: research, ethics and policy. Hum Reprod 2003; 18: 672-682.

210 European Union: Commission Directorate E, Biotechnology, Agriculture and Food: survey on opinions from National Ethics Committees or similar bodies, public debate and national legislation in relation to human embryonic stem cell research and use. VOL I in EU Member states and VOL II Countries associated to FP6 and Third Countries, 2004.

211 Expert group, a community research: 25 recommendations on the ethical, legal and social implications of genetic testing, Brussels, 2004.

212 Communication from the Commission: follow-up to the highlevel reflection process on patient mobility and healthcare developments in the European Union (COM (2004) 301 final).

213 Joint Research Center/IPTS: Report: towards quality assurance and harmonisation of genetic testing services in the EU, 2003.

214 CIOMS - Council for International Organizations of Medical Sciences: International Ethical Guidelines for Biomedical Research Involving Human Subject. Geneva, 2002.

215 Pennings G: personal desires of patients and social obligations of geneticists: applying preimplantation genetic diagnosis for non-medical sex selection. Prenat Diagn 2002; 22: 1123-1129.

216 EURORDIS: European organisation of rare diseases www.eurordis.org.

217 Nys H: Comparative health law and the harmonisation of patients' rights in Europe. Eur J Health Law 2001; 8: 317-331.

218 Nielsen L: Room for harmonization; in Donald E (ed): Conceiving the Embryo. Dordrecht: Kluwer Law International, 1996.

219 WMA - World Medical Asscociation: Medical Ethics Manual 2005.

220 Turoni G: New law forces Italian couple with genetic disease to implant all their IVF embryos. BMJ 2004; 328: 1334.

221 Pembrey ME: In the light of preimplantation genetic diagnosis: some ethical issues in medical genetics revisited. Eur J Hum Genet 1998; 6: 4-11.

222 Fasouliotis SJ, Schenker JG: Ethics and assisted reproduction. Eur J Obstet Gynecol Reprod Biol 2000; 90: 171-180.

223 Savulescu J: Procreative beneficence: why we should select the best children. Bioethics 2001; 15: 413-426.

224 Boyle RJ, Savulescu J: Ethics of using preimplantation genetic diagnosis to select a stem cell donor for an existing person. BMJ 2001; 323: 1240-1243.

225 ESHRE: The ESHRE ETHICS and Law Task Force, Taskforce 9: the application of preimplantation genetic diagnosis for human leukocyte antigen typing of embryos. Hum Reprod 2005; 20: $845-847$;

ESHRE PGD Consortium steering committee: data collection III. Hum Reprod 2002; 17: 233-246.

Guidelines for Counselling in Infertility. ESHRE Monographs, vol 2002, no 1. Oxford Journals.

226 Pattinson S: Designing donors. Cardiff Centre for Ethics, Law and Society. Issue of the Month 2003, http://www.ccels.cardiff. ac.uk/issue.html.

227 Verlinsky Y, Rechitsky S, Schoolcraft W, Strom C, Kuliev A: Designer babies - are they a reality yet? Case report: simultaneous preimplantation genetic diagnosis for Fanconi anemia and HLA typing for cord blood transplantation. Reprod Biomed $31,2000$.

228 American Society for Reproductive Medecine (ASRM): Practise committee: ovarian tissue and oocyte cryopreservation. Fertil Steril 2004; 82.

229 Practise committee: guidelines on number of embryos transferred. Fertil Steril 2004; 82: 773-774.
230 Ethics committee: child-rearing ability and the provision of fertility services. Fertil Steril 2004; 82: 564-567.

231 Golombok S, Brewaeys A, Giavazzi MT, Guerra D, MacCallum F, Rust J: The European study of assisted reproduction families: the transition to adolescence. Hum Reprod 2002; 17: 830-840.

232 Koropatnick S, Daniluk J, Pattinson HA: Infertility: a non-event transition. Fertil Steril 1993; 59: 163-171.

233 Packman WL: Psychosocial impact of pediatric BMT on siblings. Bone Marrow Transplant 1999; 24: 701-706.

234 The Danish Council of Ethics: Ethical problems concerning assisted reproduction. Part II. Anonymity and selection in the context of sperm donation, Report 2002.

235 Hardy E, Makuch MY: Gender, infertility and ART in Current Practices and controversies in assisted reproduction. Report of a WHO Meeting 2001. Geneva: WHO, 2002.

236 Bermudez MG, Wells D, Malter H, Munne S, Cohen J, Steuerwald NM: Expression profiles of individual human oocytes using microarray technology. Reprod Biomed (online) 2004; 8: 325-337.

237 Mortimer D, Mortimer ST: Quality and Risk Management in the IVF Laboratory. Cambridge (UK): Cambridge University Press, 2005.

238 Kmietowics Z: UK clinic allowed to screen embryos for rare bowel cancer. BMJ 2004; 329: 1061.

239 Simpson JL: Changing indications for preimplantation genetic diagnosis (PGD). Mol Cell Endocrinol 2001; 183 (Suppl 1): S69-S75.

240 Robertson JA: Extending preimplantation genetic diagnosis: medical and non-medical uses. J Med Ethics 2003; 29: 213-216.

241 Robertson JA: Extending preimplantation genetic diagnosis: the ethical debate. Ethical issues in new uses of preimplantation genetic diagnosis. Hum Reprod 2003; 18: 465-471.

242 Klonoff-Cohen H: Female and male lifestyle habits and IVF: what is known and unknown. Hum Reprod 2005; 11: 180-204.

243 Kovacs GT, Breheny SA, Dear Mj: Embryo donation at an Australian university in vitro fertilisation clinic: issues and outcomes. Med J Aust 2003; 178: 127-129.

244 Schiffino N, Varone F: ART policy in Belgium: a bioethical paradise?; in Bleiklie I, Goggin ML, Rothmayer C (eds): Comparative Biomedical Policy Governing Assisted Reproductive Technologies. London: Routlegde, 2004.

245 Rothmayr C, Ramjoue C: Germany: ART policies as embryo protection; in Bleiklie I, Goggin ML, Rothmayer C (eds): Comparative Biomedical Policy, Governing Assisted Reproductive Technologies. London: Routlegde, 2004.

246 Robertson JA: Protecting embryos and burdening women: assisted reproduction in Italy. Hum Reprod 2004; 19: 1693-1696.

247 Timmermans A: The Netherlands: conflict and consensus on ART policy; in Bleiklie I, Goggin ML, Rothmayer C (eds): Comparative Biomedical Policy Governing Assisted Reproductive Technologies. London: Routlegde, 2004.

248 Dubouchet J, Klöti U: ART in Spain: technocratic inheritance and modernist aspirations; in Bleiklie I, Goggin ML, Rothmayer C (eds): Comparative Biomedical Policy, Governing Assisted Reproductive Technologies. London: Routlegde, 2004.

249 The Swedish National Council on Medical Ethics: Opinion of PGD 2004

250 Schenker JG: Asssited reproductive practice: religious perspectives. RBM (online) 2005; 10: 310-319.

251 Zlotogora J: Parental decisions to abort or continue a pregnancy with an abnormal finding after an invasive prenatal test. Prenat Diagn 2002; 22: 1102-1106.

252 Serour GI: Attitudes and cultural perspectives on infertility and its alleviation in the Middle East area; in WHO (ed): Current Practises and Controversies in Assisted Reproduction. Geneva: WHO, 2002.

253 Landau R: Israel: every person has the right to have children; in Blyth E, Landau R (eds): Third Party Assisted Conception Across Cultures. Social, Legal and Ethical Pesrpectives. Jessica Kingsley Publishers: UK \& USA, 2004. 


\section{Appendix A}

\section{LEGAL FRAMEWORK}

Countries have adopted very different legal approaches to regulation of ART as well as to the jurisdiction of authority, the nature of enforcement and other particulars. Some have enacted very restrictive laws (Austria, Germany, Italy, Norway, Switzerland), some have adopted middle course regulation (Canada, France, the Netherlands, the UK, Spain) and some have rather permissive laws or no legal regulation at all, but ART issues are regulated by professional standards (Belgium, Finland, USA).

The autonomy of the professionals to practise ART varies from country to country, as well as access to treatments (see Chapter 11 of the background document). In some countries, practise of forbidden ART may lead to criminal sanctions.

Regulation at its best creates guidelines and is flexible to welcome new technologies, when they are scientifically justified. At its worse, the regulation prevents adoption of novel techniques, shifts decision-making in ART issues to courts, limits autonomy of patients and access to treatments, and leads to import and export of ART services. Professional self-regulation is often a good solution, but some of the potential applications of ART need clear guidelines, for example, limits for the use of PGD.

For regulation to be effective, there must be an authority responsible for licensing and controlling medical facilities that provide ART treatments. A frequently cited model for an effective structure of regulation is the Human Fertilization and Embryology Authority (HFEA) in the United Kingdom. ${ }^{6}$ However, it has been argued also that the system is bureaucratic and inefficient.

Whereas national regulations are very miscellaneous, the numerous different international organisations, national committees and professional associations have addressed the bioethical issues, given recommendations, guidelines and policies regarding concepts, methods and practises. Although lacking certain legal authority, this 'soft law' is still widely applied and seem to provide rather similar views on what can be considered acceptable. Soft law has significant relevance in bioethics and medical law. However, it may be difficult to recognise its potential legal status and hierarchical order of application.

These different approaches and international along with national professional and ethical guidelines will be presented below. This appendix does not aim at being exhaustive, but rather collecting mostly central European international guidelines and policies, as well present different national approaches on how issues relating to ART are being settled in various countries. The selection of countries presented is based on practical factors, mainly access to information, but not on prioritising any country over another. Therefore, also the contents of information regarding different countries vary.

\section{INTERGOVERNMENTAL ORGANISATIONS}

1. United Nations (UN) UN Convention on the Right of the Child (1990), Article 3 states that in all actions concerning children, whether undertaken by public or private social welfare institutions, courts of law, administrative authorities or legislative bodies, the best interests of the child shall be of primary consideration. The convention has been ratified almost universally; however, not by USA. According Article 7, paragraph 1: The child shall be registered immediately after birth and shall have the right from birth to a name, the right to acquire a nationality and, as far as possible, the right to know and be cared for by his or her parents. Article 8, paragraph 1: parties undertake to respect the right of the child to preserve his or her identity, including nationality, name and family relations as recognised by law without unlawful interference. Article 8 , paragraph 2: where a child is illegally deprived of some or all of the elements of his or her identity, Parties shall provide appropriate assistance and protection, with a view to re-establishing speedily his or her identity.

The physical, psychological and social well-being of children produced by ART has raised lots of discussion. In particular, the right to know one's genetic background has been debated. The donor identification has gained stronger position in the Western legislation. Whether the paragraphs of the UN Children's convention support a child's right to know its genetic background or not has been subject to different and sometimes opposite interpretations.

\section{World Health Organisation}

- Reproductive Health Strategy from 2004 mentions infertility services as a part of the five core aspects of reproductive and sexual health. Among the specific targets are reductions of maternal mortality ratio and under-5 mortality rate, as well as halting of HIV/AIDS. ${ }^{2}$

- Review of Ethical issues in Medical genetics of 2003 contains guidelines on ethical issues in medical genetics and genetic services, the items including genetic screening and testing; autonomy and informed consent; presymptomatic and susceptibility testing; disclosure and confidentiality; PND and PGD and prior and posttest counselling. ${ }^{29}$

- Based on a WHO meeting in 2002 'Current practises and controversies in Assisted Reproduction', the participants agreed upon six recommendations on the following topics: (1) infertility and ART in the developing world; (2) infertility and ART from a regional perspective; (3) recent medical developments and unresolved issues in ART; (4) social and psychological issues in infertility and ART; (5) ethical aspects of infertility and ART, and (6) national and international surveillance of ART and their outcomes. ${ }^{18}$

United Nations Educational, Scientific and Cultural Organisation (UNESCO) has also aimed to create standards in 
bioethics. It has already contributed to the formulation of basic principles in bioethics through, in particular the Universal Declaration on the Human Genome and Human Rights of 1997 and the International Declaration on Human Genetic Data of 2003. The Member States should take appropriate measures to promote the principles set out in the Declarations and encourage their implementation.

Upon the Article 17 of the Declaration on the Human Genome and Human Rights, 'States should respect and promote the practice of solidarity towards individuals, families and population groups who are particularly vulnerable to or affected by disease or disability of a genetic character. They should foster, inter alia, research on the identification, prevention and treatment of genetically based and genetically influenced diseases, in particular rare as well as endemic diseases which affect large numbers of the world's population'.

The importance of genetic counselling is emphasised in Article 11 of the International Declaration of Human Genetic Data, according to which it is 'ethically imperative that when genetic testing that may have significant implications for a person's health is being considered, genetic counselling should be made available in an appropriate manner. Genetic counselling should be nondirective, culturally adapted and consistent with the best interest of the person concerned'.

International Bioethics Committee (IBC) is a permanent committee of UNESCO established in 1998. It has published several reports relevant to the subject such as:

- Report on Genetic Screening and Testing 1994.

- Report on Human Gene Therapy 1994.

- Report on Genetic Counselling 1995.

- Report on PGD and Germ-Line Intervention 2003.

The IBC reached the following conclusions on PGD and Germ-Line Intervention in its latest report of 2003: GermLine Intervention is strongly discouraged or legally banned. PGD may be an additional option for parents at increased risk of having a child with a genetically caused disease or malformation. PGD is still considered an experimental procedure requiring highly specialised skills and a multidisciplinary approach. IBC does not make a general statement about the moral acceptability of PGD. It is recommended that PGD be limited to medical indications. Embryonic HLA typing to save a sibling with a genetic blood disease or leukaemia is considered ethically acceptable only if carried out simultaneously with PGD for the disease concerned and if mismatching of the HLA type is not considered in itself as a basis for selecting against the embryo unaffected by the disease concerned. PGD to select and implant embryos with a similar genetic disease or condition as one of the parents is considered unethical. Aneuploidy testing is considered ethically acceptable. PGD for DNA sequences should be restricted to cases involving high genetic risk and clinically severe diseases. PGD for normal physical and mental characteristics is rejected.

The IGBC (Inter-Governmental Bioethics Committee) was created in 1998, under Article 11 of the Statutes of the IBC. The IGBC is comprised of 36 Member States whose representatives meet at least once every 2 years to examine the advice and recommendations of the IBC. It informs the IBC of its opinions and submits these opinions along with proposals for follow-up of the IBC's work to the DirectorGeneral for transmission to Member States, the Executive Board and the General Conference.

UNESCO has been drafting an International declaration on Universal Norms on Bioethics, which is aimed at being finalised in the General Conference in October 2005 (www.unesco.org/bioethics).

2. Council of Europe The Council of Europe, a body set up in 1949 and now composed of 46 Member States, considers ethical issues in the field of biomedicine through a Steering Committee on Bioethics (CDBI). Its Working Party on the Protection of the Human Embryo and Foetus has published a report on 19 June 2003 of the protection of the human embryo in vitro. The report aimed to reflection on ART and PGD in particular, by outlining the various existing positions in Europe, without taking a stance on the issues raised. It shows a broad consensus on the need for the protection of the embryo in vitro. However, the definition of the status of the embryo remains an area where fundamental differences are encountered, based on strong arguments. These differences largely form the basis of most divergences around the other issues related to the protection of the embryo in vitro. Common approaches were desired to ensure proper conditions for the application of procedures involving the creation and use of embryos in vitro.

Convention for the Protection of Human Rights and Fundamental Freedoms (1950), Article 8 addresses the right to respect for private and family life, personal identity, the home and correspondence. Article 12 declares that 'Men and women of marriageable age have the right to marry and to found a family, according to the national laws governing the exercise of this right.' Some have argued referring to these articles and to Article 14 that prohibits discrimination, that infertility treatments should be available to everyone disregarding marital status, sex, age, etc. Case law of the European Court of Human Rights is limited, but in some cases other than ART, the court has ruled that countries can allocate limited health care resources, for instance, based on medical reasons, which would hence leave nonmedical purposes of ART outside this right (eg, single women, lesbian couples). This convention is very significant in practice because individuals can bring cases to the European Court of Human Rights in case they feel their rights have been breached by their governments. 
Convention for the Protection of Human Rights and Dignity of The Human Being with regard to the Application of Biology and Medicine (Convention on Human Rights and Biomedicine Oviedo 4.4.1997) is significant in the field of biomedicine, even though not so many countries have ratified it yet. (When a country ratifies certain convention, it assumes a legal obligation to implement the rights recognised in the treaty.) Biomedical convention does not constitute individual rights, but in cases pending in the European Court of Justice, also the provisions of the Biomedical convention can be appealed to. The convention of biomedicine addresses among other things the following:

Article 4, Professional Standards: Any intervention in the health field, including research, must be carried out in accordance with relevant professional obligations and standards.

Article 12, Predictive genetic tests: Tests that are predictive of genetic diseases or which serve either to identify the subject as a carrier of a gene responsible for a disease or to detect a genetic predisposition or susceptibility to a disease may be performed only for health purposes or for scientific research linked to health purposes, and subject to appropriate genetic counselling.

Article 13, Interventions on the human genome: An intervention seeking to modify the human genome may only be undertaken for preventive, diagnostic or therapeutic purposes, and only if its aim is not to introduce any modification in the genome of any descendants.

Article 14, Nonselection of sex: The use of techniques of medically assisted procreation shall not be allowed for the purpose of choosing a future child's sex, except where serious hereditary sex-related disease is to be avoided.

Article 21, Prohibition of financial gain: The human body and its parts shall not, as such, give rise to financial gain.

A new additional protocol to the Convention on Human Rights and Biomedicine concerning biomedical research was introduced in June 2004. The protocol covers full range of research activities in the health field involving interventions on human beings. It does not apply to research on embryos in vitro, but it does apply to research on foetuses and embryos in vivo. General provisions include that research may only be undertaken if there is no alternative of comparable effectiveness. Article 18 sets conditions on research during pregnancy or breastfeeding: research on a pregnant woman, of which she or her embryo, foetus or future child do not have direct benefit, may only be undertaken, (1) if the research has the aim of contributing benefit to other women in relation to reproduction or to other embryos, foetuses and children; (2) research of comparable effectiveness cannot be carried out on women who are not pregnant, and the research entails only minimal risk and minimal burden. New developments are addressed in Article 24.
Working Party on Human Genetics has made a working paper, under the responsibility of CDBI, with a view to the elaboration of an additional Protocol concerning genetics to the convention on Human Rights and Biomedicine. It was open for comments till 30 April 2003. According to the information received form the Treaty Office, CDBI started, at its plenary meeting in October 2004, the examination of the first part of the draft Protocol, which concerns general provisions and the health field, prepared by the Working Party taking into account the comments received on the working document. It agreed to focus the Protocol on genetic tests. According to the document, however, the protocol is not supposed to extend to the applications of genetics to the human embryo and foetus or any biological material derived from them. In 2005, discussion on this part of the draft Protocol will continue at the level of the CBDI with view to its finalisation.

3. European Union Charter of Fundamental rights of the European Union, approved by the member states in 2000 was further accepted as a part of the EU Constitutional Convention in June 2004. It is not yet in force. The Charter has provisions applicable to biomedicine and research, such as personal integrity; informed consent requirement; prohibitions against the use of methods for genetic enhancement and selection of people; and ban on deriving economical beneficence from human body or its parts and reproductive cloning.

European Union has not adopted directive or other regulation on particularly applicable to ART. Some parts of the following directives shall be applied also when using ART with respect to safety of the use of gametes and performance of tests.

(1) 'Tissue Directive'

Directive 2004/23/EC of the European Parliament and of the Council of 31 March 2004 on setting standards of quality and safety for the donation, procurement, testing, processing, preservation, storage and distribution of human tissues and cells, 'the Tissue Directive', entered into force on 7 April 2004. The member countries shall implement it by 7 April 2006. The objective of the directive is namely to ensure high level of health protection and to prevent transmission of diseases by human cells and tissues. According to its Recital 7, the Directive is also, among other things, applied to reproductive cells, foetal tissues and cells as well as adult and embryonic stem cells. Recital 12 allows Member States make own decisions concerning the use or nonuse of any specific type of human cells. According to Recital 18, tissue and cell application should be founded on the philosophy of voluntary and unpaid donation, anonymity of both donor and recipient. However, in Recital 29 is stated that Member States could legislate otherwise in exceptional cases, notably regarding donation of gametes. Recital 22 refers to the Charter of Fundamental Rights of the European 
Union and Convention on Human Rights and Biomedicine and claims to respect them.

Article 8 sets a requirement that all tissues and cells meant by the Directive shall be traceable. The data shall be coded and kept for a minimum of 30 years after clinical use. Article 14 concerns data protection and confidentiality and states that all data, including genetic information, collated within the scope of the Directive and to which third party have access, shall be rendered anonymous so that neither donors nor recipients remain identifiable. Article 9 stipulates the import and export of tissues and cells. Only licensed establishments can be used, traceability shall be ensured and equal standards of quality and safety requirements shall be met as in the Directive. According to Article 19, all donations of tissues and cells shall be tested in accordance with the requirements referred to in Article 28(e) and selection and acceptance of tissues and cells shall comply with the requirements referred to in Article 28(f).

An annex of the Directive sets following requirements for information to be given to the donor:

1. The person in charge of the donation process shall ensure that the donor has been properly informed of at least those aspects relating to the donation and procurement process outlined in paragraph 3 of the Directive. Information must be given before the procurement.

2. The information must be given by a trained person able to transmit it in an appropriate and clear manner, using terms that are easily understood by the donor.

3. The information must cover the purpose and nature of the procurement, its consequences and risks; analytical tests, if they are performed; recording and protection of donor data, medical confidentiality; therapeutic purpose and potential benefits; and information on the applicable safeguards intended to protect the donor.

4. The donor must be informed that he/she has the right to receive the confirmed results of the analytical tests, clearly explained.

5. Information must be given on the necessity for requiring the applicable mandatory consent, certification and authorisation in order that the tissue and/or cell procurement can be carried out.

The Tissue Directive requires establishment of special authority for inspection, licensing and accreditation; a quality system approach, proper expertise and a monitoring system for adverse incidents and reactions. The criteria for these will be set in the following documents:

\section{(2) 'Technical Documents'}

The Tissue Directive empowers the Commission to establish and update technical requirements in relation to quality and safety of human tissues and cells. The Commission conducted an open consultation for this and has prepared two documents, which have not been adopted yet. The latest outline of the first document dates from November 2004. Both documents are still in a consultation phase. The Council of Europe and WHO were consulted to ensure coherence between guidelines.

The first document aims to cover all human cells and tissues and all manufactured products derived from them, which are used for application to the human body, during the first phases of the process - donation, procurement and testing - in order to ensure their quality and safety.

A second document will address the technical requirements for processing, preservation, storage and distribution of human tissues and cells and will incorporate the criteria for accreditation/designation/authorisation/licensing of tissue establishments, the quality system requirements, the tissue coding requirements and the requirements for adverse event reporting.

The future Directive will introduce definitions of reproductive cells, partner donation and direct use and set technical criteria for the assessment of donor eligibility. It contains minimum testing levels to be carried out on potential donors and requires thorough examination of the health and background of potential donors of reproductive cells. The testing device has to be CE marked. In the case of partner donation for direct use, selection criteria and tests are not applied, whereas if the cells are not to be used directly, several serology tests must be carried out. According to Section 'The main indications of PGD' of the outlined draft, the use of reproductive cells from third party requires immense testing and genetic screening depending on the donor's medical, familial or ethnical background. For instance, genetic screening for autosomal recessive genes known to be prevalent in the donor's ethnic background and an assessment of the risk of transmission of inherited conditions known to be present in the family shall be carried out, after obtaining consent and providing complete information, in accordance with the requirements in force in Member States. Complete information on the associated risk and on the measures undertook to its prevention shall be communicated and clearly explained to the recipient.

(3) In vitro diagnostic directive 98/79/EC aims at protecting safety of the in vitro medical devices and obliges mainly the manufacturers by setting the essential requirements devices must meet before being placed on the market. The directive refers to ethical provisions of Biomedical convention by stating in the Article: 'For the purposes of this Directive, the removal, collection and use of tissues, cells and substances of human origin shall be governed, in relation to ethics, by the principles laid down in the Convention of the Council of Europe for the protection of human rights and dignity of the human being with regard to the application of biology and medicine and by any Member States' regulations on this matter'.

(4) Proposal of Directive on Services (COM (2004) 2) is at present largely debated among the member states (Spring 
2005). First drafts aimed at ruling also of the free movement of healthcare services and reimbursement issues, but a suggestion has been made to remove healthcare services from the scope of application. The patient mobility and the access to health care within EU have been addressed in Chapter 11 of the background document.

The issues of assisted reproduction have not been much addressed to within the European Union. Several committees under the European Commission and the European Parliament have taken following initiatives in reports and opinions, which might have certain impact indirectly in the field of ART and genetics as well:

- European Parliament Temporary Committee on Human Genetics and Other New Technologies in Modern Medicine (November 2001) identified certain questions relating to PGD and other testing, but did not end up in any conclusion. (Report on the ethical, legal, economic and social implications of human genetics http:// europa.eu.int/comm/research/biosociety/pdf/pe_genetics. pdf)

- A multidisciplinary expert group in the field of law, philosophy, ethics and medicine, invited by the European Commission, has released a set of 25 recommendations on human genetic testing for medical purposes in 2004. http://europa.eu.int/comm/research/conferences/ 2004/genetic/pdf/recommendations_en.pdf

- Commissioner David Byrne launched a reflection process for a new EU Health Strategy in July 2004 'Enabling Good Health for all'.

The Joint Research Centre (JRC) is a body of the European Commission providing scientific and technical advice to support EU policies and the Institute for Prospective Technological Studies (IPTS) is one of its seven scientific institutes. JCR/IPTS has released a report on quality standards of genetic testing in Europe in 2003 (Report: Towards quality assurance and harmonisation of genetic testing services in the EU).

European Group on Ethics in Science and New Technologies (EGE) is an independent, pluralist and multidisciplinary body which advices the European Commission on ethical aspects of science and new technologies in connection with the preparation and implementation of Community legislation or policies.

\section{National regulation \\ European countries \\ Austria}

Austrian Federal Law of 1992 (Serial 275) regulating Medically Assisted Procreation (The Reproductive Medicine Law), and Amending the General Civil Code, is restrictive. Its central principle is that reproductive medicine is acceptable only within a stable heterosexual relationship for the purpose of reproduction. The law provides that embryos can be used only for implantation in the woman whose oocytes are being used and cannot be used for other purposes. The donation of embryos or gametes is explicitly prohibited, excluding semen donation in partner IVF. PGD is not allowed currently. Two alternative approaches to allow the use of PGD were presented in an opinion of Bioethics committee in July 2004: the other would limit the use of PGD only for testing of nonviability of an embryo when treating infertility, and the other testing of severe genetic illnesses on a case-by-case consideration of an authority. Austria has not signed the Convention on Human Rights and Biomedicine.

\section{Belgium}

In Belgium, the professional codes of conduct and ethical principles of hospitals and clinics have for long time ruled the practise. The law on embryo research was adopted in 2003, but ART remained unregulated excluding PGD which is allowed for therapeutic purposes. ${ }^{244}$ Most ART are being practised.

According to Article 3 of the Law on embryo research, research on embryos is allowed if following conditions are met: (1) research has to have a therapeutic aim or has to increase knowledge about reproductive fertility, transplantation, disease treatment, (2) is based on recent research findings and fulfils demands of correct methodology of medical research, (3) is performed in an accredited lab associated to a university care unit, (4) is carried out under the supervision of a specialist medical doctor or scientist, (5) is performed before day 14 and (6) there are no other methods of research. Article 4 states that (1) creating embryos for research (surnumerary embryos) is forbidden, unless the aim of the research cannot be reached by other means, (2) ovarian stimulation is allowed if the woman is of age, has given written consent and the stimulation is performed as scientifically justified. Under Article 5, it is forbidden (1) to implant human embryos in animals or to create chimera or hybrids, (2) to replace embryos that have been used for research in humans, unless the research was performed with a therapeutic aim for the embryo or when an observation method that does not harm the embryo has been used, (3) to use embryos, gametes or embryonic stem cells for commercial ends, (4) to perform research or treatment with a eugenic aim and (5) to perform research or treatment aimed at sex selection, unless it is for sexlinked diseases. Reproductive cloning is forbidden. Research on embryos is subject to approval of local ethical committees and the Federal Commission for medical and scientific research. The contents and procedures of informed consent are described in the law. The law also established a Federal Commission for medical and scientific research, consisting of four MDs, four PhDs, two lawyers, four experts in ethical and social sciences, to which every researcher must send an annual report. Violations of the law are subject to penalty.

Belgium has not signed the Convention on Human Rights and Biomedicine. 


\section{Denmark}

The Danish rules governing ART are laid down in the Act on Assisted Reproduction of 1997 (Lov om kunstig befrugtning) as well as various guidelines and executive orders, primarily the National Board of Health's guideline from September 1997, the Guideline on assisted reproduction and other reproduction-promoting treatment - for Danish doctors. The legislation applies solely to the treatment provided by a doctor or under the responsibility of a doctor. In addition, the Children Act of 2002 contains some relevant provisions regarding the assignment of paternity in the case of assisted reproduction.

Most ART is allowed in Denmark at present. Nevertheless, surrogacy and ART after the age of 45 in the woman is not allowed. Doctors are not allowed to perform insemination of lesbian couples. Oocyte donation is restricted to women undergoing IVF owing to their own infertility. Gamete donors are guaranteed anonymity, if insemination or oocyte donation is provided by professional medical services. The issue of whether a child has a right to know its genetic parents has been actually debated also in Denmark. The Danish Council of Ethics has published several reports on issues relating to ART and genetics, inter alia, it has addressed thoroughly the issue of anonymity and selection in the context of sperm donation in its report of 2002. ${ }^{234}$

The National Board of Health has issued guidelines of the tests a donor has to undergo before use of his sperm (tests include HIV, syphilis and gonorrhoea, and hereditary disorders that can be detected). Donor's phenotype can be considered when choosing sperm for recipient to allow preferences of ethnic and familial similarity. Private sperm banks have also used other personal counter-selection criteria, such as personality, motives and physical appearance, which are not supported by legislation, though.

A Health Technology Assessment report, covering technological, patient-related, ethical and health economic aspects of PGD was published in 2002 under the auspices of the National Board of Health. ${ }^{10}$ After law amendment in 2004, the National Board of Health can give a license to use PGD for HLA matching upon consideration case by case.

PGD for severe inherited diseases and for aneuploidy screening is allowed within the framework of a research protocol, approved by the research ethics committee system. A more general allowance is presently under consideration.

Embryonic stem cell research is allowed, and stem cell lines may be produced from excess embryos from IVF treatments, after ethical permission and informed consent from the couple.

Denmark has ratified the Convention on Human Rights and Biomedicine in 1999.

\section{Finland}

In the absence of legislation on ART, Finland has until now offered a very high level and broad access to ART subject to professional ethical standards, under license of the National Authority for Medicolegal Affairs (TEO). However, the new public healthcare project will bring some limitations to the access in the public sector or to reimbursement in the private sector (prognosis, age of woman, number of cycles), as all the treatments are as of March 2005 subject to a specific relevance criteria (point award system). Similar system is used at least in the New Zealand. Moreover, the largely debated governmental proposition of 2002 for legislation on assisted reproduction will be presented again to the Parliament during 2006. The proposal was withdrawn after the first presentation, as certain controversial issues could not be resolved, mainly the right of homosexuals and single women to get fertility treatment, and donor identity. Under the proposal access to treatments are targeted for involuntary childlessness owing to medical reasons and prevention of certain severe hereditary illnesses (PGD). Also, single women and lesbian couples are suggested to have a right to ART. Donor anonymity will be abolished. The law would not rule of the techniques as such. Basically all the treatments would be allowed, as long they are scientifically justified. Same donor could be used five times plus siblings.

Finland has signed, but not ratified, the Convention on Human Rights and Biomedicine.

\section{France}

In France le code civil and le code de la santé publique contain provisions of the bioethical issues. ART-related issues became regulated by the Laws on Bioethics passed in 1994. Legal framework in France is considered to be situated in the middle course among other European laws. A recent large amendment to the current law (Loi ${ }^{\circ} 2004-$ 800 du 6 août 2004 relative à la bioéthique) stipulates very thoroughly on ART. The authorities under this law are Comité consultatif national d'éthique pour les sciences de la vie et de la santé (CCNE) and a new 'Agence de Biomedicine' that substitutes the former 'Etablissement francais des greffes'. Annual reports from centres are required to be submitted to the Agency, which has at its aim to oversee ART issues. CCNE has issued several relevant opinions on the subject matter.

The law permits many forms of ART and related techniques, but under the surveillance of Agence de Biomedicine and only to heterosexual couples on therapeutical medical grounds. The law can be considered a predecessor in an ethically difficult situation of PGD-HLA, as it enables 'saviour sibling' with a consent of l'Agence de Biomedicine (Article L. 2131-4-1). PGS, however, is not allowed. The new law allows furthermore research on surplus embryos with no implantation potential under the consent of the parents. Both therapeutic and reproductive cloning remained prohibited. Surrogacy is forbidden as well. Gamete and embryo donations are allowed. Social security policy covers the main costs of the necessary ART treatments. 
France has signed, but not ratified, the Convention on Human Rights and Biomedicine in 1997.

\section{Germany}

In Germany ART are governed by the Embryo Protection Act of 1990 (Embryonenschutzgesetz, EschG). This law declares different types and uses of ART to be criminal and thereby formulates very restrictive policies. EschG fully prohibits oocyte donation, embryo donation and declares the transfer of more than three embryos to a woman within a cycle to be a punishable offence. It also prohibits the creation of an embryo for any other purpose than transferring it to a woman from whom the oocyte comes in order to induce pregnancy. Any research on embryos and totipotent cells is criminalised. Deriving stem cells from embryos for research purposes is forbidden by EschG.

The Stem Cell Act (Stammzellengesetz, StZG) was adopted in 2002. Regulation is somewhat controversial, as EschG forbids deriving the stem cells from embryos, but StZG allows their import in certain conditions for research purposes.

The German Medical Chamber has given the Guidelines on Assisted Reproduction, which have legally binding character.

Consequently, the German regime regarding ART can be considered very restrictive. The protection of the embryo is emphasised. The access to ART is limited to marital status and sexual orientation, on a case-by-case basis, to stable unmarried couples. Insurance coverage for married couples is relatively broad, but denied for unmarried couples, if granted access. ${ }^{245}$

Manipulation of the embryo after syngamy of the two pronuclei is not allowed in Germany. The law as such does not forbid PGD, but the way it is interpreted at the moment prevents performance of PGD on cleavage stage embryos and blastocysts, whereas PGD on polar bodies is applied in Germany. Whether polar body biopsy violates the meaning of the law is debated. ${ }^{83}$ The German National Ethics Committee has addressed the issue of PGD in its opinion of 2003. ${ }^{84}$ Recent surveillance indicates that the public opinion is in favour of allowing PGD in Germany. ${ }^{125}$

Germany has not signed the Convention on Human Rights and Biomedicine.

\section{Greece}

Greece has since 2002 a legal framework on ART. The first law (Law 3089/23-12-2002) provides broad framework, wheres the latest one (Law 3305/27-1-2005) provides for more detailed framework: ART should be applied taking into account mainly the best interests of the child to be born; the upper age limit for the woman is 50 years; informed consent is mandatory after providing detailed information on all aspect of treatment; the number of embryos to be transferred is limited: in women up to 40 years up to three embryos and over 40 years up to four embryos. Furthermore, the law allows PGD, PGS and emrbryo research after the approval from the Authority. An independent ART authority is established to oversee the whole field. It audits and licences the ART Centres and Cryobanks, renews the licences, gives permission for specific techniques and research, etc. For violations of the two laws, there are specific penalties. ART will be covered by the state insurances.

Greece has ratified the Convention on Human Rights and Biomedicine in 1998.

\section{Italy}

ART procedures have experienced 20 years of activity in this field, with more than 10000 couples who have been treated. Although a wide debate in the field was present from long time no specific law was present and only in 2004 , the first law on medically assisted reproduction has been approved by the Italian parliament. This law is said to be the most restrictive in Europe. The law has been criticised both in and outside of the country because of its excessive concern with the status of embryos in comparison to the interests of women and infertile couples. ${ }^{246}$ Donation of embryos and gametes is banned, as well as cryopreservation. A maximum of three oocytes can be fertilised and every embryo, without testing, has to be transferred into uterus regardless of the quality of the embryo. PGD is absolutely prohibited. In this new situation, nonsterile couples at high genetic risk have started to seek PGD abroad. ${ }^{126,220}$ The ART procedures performed within public hospitals follow the same rules of the other medical treatments within the National Health System. The centres needs to fulfil the accreditation criteria according to the national law, but are more specifically regulated by Guidelines on Assisted Reproduction approved by each regional government.

The existing IVF embryos in frozen storage in Italy will be put up 'for adoption' if unclaimed, and a storage facility has been set up as a national centre in Milan.

A complete change of the law was asked in the spring 2005, but the national consultation 'referendum' did not reach the number of votes needed, and therefore the request has been rejected. A national debate is still present and there is quite a wide opinion than less radical changes could improve the present law.

Italy has signed, but not ratified, the Convention on Human Rights and Biomedicine in 1997.

\section{The Netherlands}

The Netherlands has several laws and decrees to rule ART issues: Law on special medical acts (Wet op Bijzondere Medische Verrichtingen WBMV) rules that certain medical acts are forbidden, unless the Minister of Public Health, Welfare and Sports granted a licence. IVF, for instance, is subject to a licence (Decision specifying WBMV). Planning Decree on In Vitro Fertilisation of 1998, based on WBMV, set the maximum of 13 IVF centres in the country and determines that IVF should be performed according to the Guideline 'indication IVF' of the Dutch Association of 
Obstetrics and Gynaecology of 1998. Moreover, IVF should follow the protocol as referred to in Article 2 of the Embryo Act of 2002. The Embryo Act sets rules for embryos/ gametes used for own pregnancies and addresses the question what can be carried out with gametes and embryos when these are not used for own pregnancies (donation, scientific research, property and rights on gametes and embryos). Some acts are specifically forbidden, for instance, reproductive cloning, the creation of chimeras, sex selection in the absence of medical indication and changing the nucleus of germ cells, which will be used to establish a pregnancy (germline gene therapy). The Embryo Act only specifically prohibits changing the nucleus of germ cells, therefore leaving open the possibility of changing DNA outside the nucleus (mitochondria).

The Embryo Act prohibits the creation of embryos for the sole purpose of research (Article 24a). However, this prohibition may be temporary because Article 33 states that Article 24a will expire on a date which will be set by Royal Decree. At the time Article 24a expires, Article 11 will automatically enter into force, which would prohibit research on embryos specifically created for research, except in cases where research is intended to increase knowledge on infertility, ART, genetic diseases or transplantation (thus a limited prohibition). Within 5 years of entering into force of the Embryo Act, a proposal for the above-mentioned Royal Decree will be made. At the moment, such proposal has not yet been made, and it is not to be expected to be made in the very near future owing to arrangements made between the coalition partners of the current government. Therefore at the moment a full prohibition is in force.

Planning Decree on Clinical Genetic Investigation and Genetic Counselling is also based on the WBMV and determines that PGD can only be performed with licence of the Minister. For the time being, the University Hospital Maastricht is the only centre that is allowed to perform PGD. The Decree contains specific procedural instructions. The Central Committee on Research involving Human Subjects (CCMO) provides advice on procedures and acts as a monitoring authority. A positive judgement of the CCMO is needed for PGD, subject to conditions set by Embryo Act.

As of June 2004, the Netherlands deserted donor anonymity and AID children will at the age of 16 have the possibility to find out who their donors are. With regard to how many children can be conceived by one donor, the decision is left for donors. Most donors tend to prefer to donate to four or five women plus siblings.

Medical autonomy is limited through licensing, monitoring and reporting. The ART policy is considered at a medium position on the dimensions of autonomy and access. $^{247}$ The Dutch Association of Obstetrics and Gynaecology provides guidelines to be followed. The Minister of Public Health, Welfare and Sports, the Central Committee on Research involving Human Subjects (CCMO) and the Health Council are authoritative bodies advising, licensing and monitoring medical professionals and institutions operating in the field, for example, by annual reports and other procedures. Varying arrangements have been drawn up concerning the financial aspects of IVF treatment.

The Netherlands has signed the Convention on Human Rights and Biomedicine in 1997.

\section{Norway}

Law on Medical Application of Biotechnology of 1994, revised in 2003 (Lov om humanmedisinsk bruk av bioteknologi $\mathrm{m} . \mathrm{m}$ ), is considered rather restrictive. This law also regulates gene therapy and reproductive technology. The access to treatments is limited to medical reasons and prevention of severe diseases. Marital status or stable heterosexual relationship is required. Embryos and oocytes cannot be donated, but sperm donations are allowed. Surrogates are not allowed. PGD is only allowed in cases of serious hereditary sex-linked disease with no available treatment. However, there is a possibility to apply for exception from the law and recently a couple was allowed treatment including both PGD and tissue matching in order to have matching stem cells for a seriously sick sibling.

The Norwegian Biotechnology Advisory Board (www.bion.no) has evaluative and advisory role to the government on all cases involving use of biotechnology. ICSI has in 2003 been permitted by law and MESA/TESE is permitted on a temporary basis after application to the Health Authority. Research on embryos or embryonic stem cells is not allowed. As of January 2005, donor anonymity is abolished and the child has a right to know the identity of the donor when he/she reaches the age of 18. Each donor can give rise to six children.

Norway has signed the Convention on Human Rights and Biomedicine in 1997.

\section{Portugal}

ART issues in Portugal are regulated by professional standards, but a law proposal for a broad legislation is planned, which would include a creation of a technical Authority on Human Reproduction and Embryology.

At the moment, ART treatments are offered to any adult $(\geqslant 18$ years), heterosexual and stable (economically, socially and psychiatrically) couple, independently of the legal marriage status. All main central public hospitals offer ART treatments (five in Porto, one in Coimbra and two in Lisbon), which are covered by public funds up to four trials. Public funds, however, do not cover medication used for ovarian controlled hyperstimulation. Public hospitals refuse access to women with $>39$ years. There is no insurance covering of ART in Portugal.

All centres, public and private, are requested to comply with ESHRE Guidelines of good medical and laboratory ART practices. All centres, public and private, are requested to send each year a full report on procedures, patients, 
cycles of treatments, laboratory results and clinical outcomes. These data are published each year and sent to ESHRE.

Provided services compose a broad assortment, including ICSI and PGD/PGS and also ooplasm transplantation for mitochondrial diseases. All couples will have a full diagnostic procedure before a treatment cycle is initiated. These include the determination of the infertility causes and all infectious transmissible agents. Karyotypes of the couples will be detected before ICSI cycles. Y-microdeletions are screened in case of severe oligozoospermia or secretory azoospermia. In case of CBAVD, CFTR screening is performed and, if positive, the wife is also tested, and if both positive PGD is offered. In special cases, sperm is studied for screening aneuploidy (FISH), apoptosis, imprinting defects and CAG repeats, whereas transmission electron microscopy is performed if $>90 \%$ immobility. Genetic testing is performed under patient-informed consent. Genetic testing procedures are annually licensed by the European Molecular Genetic Quality Network (EMQN).

Portugal provides with oocyte, sperm and embryo cryopreservation, but also long-term embryo culture in order to enable blastocyst transfer, which is reported to decrease the number of frozen embryos, increase the pregnancy rate and decrease multiple pregnancies (70\% single, 20\% double).

Ovary and testicle tissue cryopreservation is offered before malignancy treatments or in case of TESE.

The rules for embryo transfer are the following: In the last 4 years (2000-2003), only 7\% of the cycles had cryopreserved surplus embryos. This low number is owing to a soft ovary hyperstimulation (mean: 6-8 oocytes/cycle). All embryos were later requested for replacement.

Even though sperm and oocyte donations belong to both public and private current practice, couples have went to Spain for DI, because the Portuguese state has not yet released the necessary funds for building a Portuguese tissue bank. General rules follow matching based on skin colour, height, hair, eyes and blood $\mathrm{ABO} / \mathrm{Rh}$ typing. Donors are checked by a full personal and family history (absence of all main known diseases, including oncologist and psychiatric), absent history of alcohol, tobacco and drug abuse, and absence of infectious transmissible agents. In general, the same donor can be used between five to 10 times.

Embryo donation is not accepted in the guidelines, but in the future law it will be allowed. Surrogacy is not accepted in the guidelines, but in the future law it may be allowed for women with congenital malformations and hysterectomy.

Treatment of single parents and homosexuals (men and women) and posthumous or postmenopausal conception are presently not accepted in the guidelines, and there has been no consensus to accept these couples in the next law either.

Pregnancy termination is allowed by law if the fetus has confirmed anatomic or genetic anomalies, or in case of violation.

It is agreed that creation of human embryos for research purposes is forbidden.

Research on unviable embryos is authorised, under patient informed consent.

It is agreed that reproductive cloning should continue to be forbidden.

Therapeutic cloning and the use of surplus blastocysts to derive embryonic stem cells for transplantation will be allowed in the next law.

All centres have closed to access to data bases, with full protection of personal data. All products, materials and cells have a traceable system.

Portugal has ratified the Convention on Human Rights and Biomedicine in 2001.

Spain

Spain was one of the first countries to adapt a law on ART and on the donation and use of human embryos and foetuses or their cells, tissue and organs in 1988, which was also rather liberal despite a strong catholic tradition (Ley sobre Técnicas de Reproducción Asistida: LTRA). The law was revised in 2003 (45/2003). Basically, any woman can have access to treatments and a variety of techniques are available. ${ }^{248}$ Reimbursement is limited to fertility criteria, however. Public hospitals have ART units, although a big amount of services are provided by private clinics. Spain has been target to reproductive tourism owing to its liberal policy. ${ }^{126}$ However, difficulties of integrating new technologies limit the available treatments in practise. Spain along with Belgium and Greece are the only European countries to allow posthumous insemination. PGD is allowed.

The Spanish legislation authorises research using unviable embryos, so that the main problem regards the concept of viability. The discussion focuses on whether surplus cryopreserved embryos should be considered unviable or not, when they have passed their legal date for use and have been discarded for any parental project, as their chances of developing in a liquid nitrogen tank are nil. The National Commission on Assisted Reproduction and the Observatory of Bioethics and Law, as well as many scientific groups, have all declared themselves in favour of this option. The Spanish government has in 2004 formally approved a decree clarifying the country's laws on human embryonic stem (ES) cell research.

The government of Spain will try to approve before the end of 2005 a new law on assisted reproduction in which PGD for tissue typing, late-onset diseases and susceptibility genes would be accepted, as well as research with spare embryos.

Spain has ratified the Convention on Human Rights and Biomedicine in 1999. 


\section{Sweden}

There is no special licensing body of IVF clinics in Sweden. Half of the clinics are private, and half officially funded.

Sweden has several laws regulating ART: the Act on Insemination of 1984 (Lag om insemination), the Act on IVF of 1988 (Lag om befrukting untanför kroppen) and the Act on procedures in research and use of fertilised human ova of 1991 (Lag om åtgärder i forsknings - eller behandlingssyfte med befruktade ägg från människa).

Another law that influences ART is the law from 2003 about tissue banks, stating that all cells, tissue, etc that are traceable and kept for more than 2 months, must be registered in a national register. The Swedish National Board of Health and Welfare (Socialstyrelsen) has also issued several guidelines concerning assisted reproduction and genetics. For instance, only one embryo shall be implanted, in 'special circumstances' two (eg, older women, several cycles performed without pregnancy).

Since 2003 oocytes and sperm may be donated to be used in IVF treatment, although only at University Hospitalbased clinics (ie, not at private). Embryo donation is not allowed. Since 1985, the donors are identifiable to children when they reach maturity. This has caused a variable shortage of donors. Each donor may give rise to six children plus siblings. Some patients still seek treatments abroad, mainly in the Denmark and also in Finland. A law amendment in summer of 2005 allows DI also to lesbian couples. Until that, access to ART treatments was limited to stable heterosexual couples.

The Swedish National Council on Medical Ethics (SMER) issued an opinion of PGD in The Swedish national council on medical ethics, ${ }^{249}$ when new indications for PGD kept on appearing. PGD has been performed in two clinics, one in Stockholm and one in Gothenburg. The Swedish Parliament set rather restrictive guidelines on PGD in 1995, which since then has ruled the practise. The indication criteria have been limited to serious, life threatening and incurable diseases. The Council now proposes a framework law governing PGD, which would follow the existing guidelines of the Parliament. This proposal suggests a more liberal use of PGD for medical purposes, but PGS only for research purposes, after permission from an ethical committee.

Embryonic stem cell research is allowed, and stem cell lines may be produced from excess embryos from IVF treatments, after ethical permission and informed consent from the couple.

Sweden has signed, but not ratified, the Convention on Human Rights and Biomedicine in 1997.

\section{Switzerland}

The Swiss Reproductive Medicine Act of 1998, in force since January 2001 (Fortpflanzungsmedizingesetz (FmedG)/Loi fédérale sur la procréation médicalement assistée (LPMA)/Legge sulla medicina della procreazione
(LPAM) of and Reproductive Medicine Ordinance of 2000, in force since January 2001 (Fortpflanzungsmedizinverordnung/Ordonnance sur la procréation médicalement assistée/Ordinanza sulla medicina della procreazione), govern the conditions under which medically assisted human reproduction is permissible in Switzerland. The Act declares the welfare of the child to be its primary principle, and requires every couple seeking treatment to undergo a comprehensive counselling process.

The fertilisation of an oocyte outside the female body is used extensively to start pregnancy. No more than three embryos may be produced per treatment cycle in order to prevent large-scale multiple pregnancies and the creation of surplus embryos. Data on the sperm donor are held at the Federal Civil Registry Office and must be made available to children conceived using this process.

The practice of medically assisted reproductive techniques requires a licence, as does the conservation of gametes and fertilised ova. Persons holding a licence are subject to a reporting obligation and the cantons must also maintain constant supervision over their activities.

The law prohibits preservation of embryos, oocyte donation, surrogacy and in vitro genetic testing of embryos (PGD). Polar body diagnosis is not forbidden, though, and a parliamentary committee has mandated the Swiss government to examine whether the interdiction of PGD should be changed. The improper collection of embryos and their development outside the female body beyond the time at which they can become attached to the uterus lining, as well as germ gene therapy (intervention to alter the genetic composition of gamete and embryos) and cloning are also punishable.

A recent amendment in the law allows stem cell research with surplus embryos.

A National Ethics Commission, appointed by the Federal Council, monitors scientific developments in the field of human medicine and draws up recommendations for medical practice. The commission exists in a purely advisory capacity. Its secretariat is attached to the Swiss Federal Office for Public Health.

Switzerland has signed, but not ratified, the Convention on Human Rights and Biomedicine in 1999.

\section{The United Kingdom}

The Human Fertilisation and Embryology Act of 1990 regulates some reproductive issues, such as donation and storage of gametes and embryos as well as creation and use of embryos outside the woman's body for both treatment and research purposes. Any use or storage of embryos and gametes conducted outside the terms of a licence is criminalised. The Act stipulated of the formation of the Human Fertilisation and Embryology Authority (HFEA), which is a statutory regulatory and licensing body. HFEA has published the 6th edition of very specific Code of Practise regarding the practise of ART (http://www.hfea.gov. 
uk/HFEAPublications/CodeofPractice). The Act will be reviewed by the Government in order to bring it up to date with the new techniques (Hansard Society's report 2004/UK House of Commons/Science and Technologies Committee).

The HFE Act has initiated the HFEA to take into the Code of Practice provisions on how to consider the welfare of the child, for example, parental capacity to bring up a child and social and psychological environment, etc. The United Kingdom removed the anonymity of the donor in April 2005, allowing thus a child to know its biological parent when reaching maturity. Semen donated before April 2005 from anonymous donors may be used for treatment until April 2006. This has caused a lot of concern as is feared it will lead to a shortage of sperm. As of April 2006, treatment with anonymous sperm is no longer allowed.

In the UK, for instance, only minority of the IVF clinics are licensed to freeze oocytes, because few requests have been received.

The HFEA has in November 2004 launched a public consultation on sperm, egg and embryo donation. The online web consultation (www.hfea.gov.uk), entitled 'The Regulation of Donor Assisted Conception', is seeking views on issues such as limits on the number of children per donor, how donor's characteristics should be matched with patients, and how much compensation donors should be paid. One proposal outlined in the document is to increase the compensation payment made to oocyte donors, up to as much as $£ 1000$. At the moment they receive $£ 15$, plus 'reasonable expenses', the same amount as sperm donors. The consultation forms part of the HFEA's ongoing SEED (sperm, egg and embryo donation) review, which includes a survey of UK clinics and a review of current scientific and clinical evidence in this area. Over 37000 children have now been born in the UK following donor-assisted conception.

Any clinic wishing to carry out PGD must first obtain a licence from the HFEA. The HFEA issues licences for PGD where the embryo is at significant risk of developing a serious condition. All PGD applications are sent out to a minimum of two peer reviewers and decisions are taken by HFEA licence committees who consider all the scientific, legal, ethical and medical information. On July 2004, HFEA decided to extend the rules allowing embryos to be tested in order for families to have a child who could be a tissue match for a seriously ill brother or sister. According to Suzi Leather, HFEA Chair 'Our review of the evidence available does not indicate that the embryo biopsy procedure disadvantages resulting babies compared to other IVF babies. It also shows that the risks associated with sibling to sibling stem cell donation are low and that this treatment can benefit the whole family.'

HFEA has issued a preimplantation genetic diagnosis (PGD) licence for Familial Adenomatous Polyposis Coli (FAP) to the Assisted Conception Unit at University College
Hospital, London. HFEA has informed of the fresh report Assisted reproduction: a safe, sound future issued on 23 November 2004 by the Medical Research Council (MRC). The HFEA approached the MRC in 2002 to review the current knowledge of IVF and its possible health effects and provide advice on what further research is necessary.

Human Genetics Commission (HGC) has in 2004 started preparing a report to the UK Government and arranged an open consultation on genetics and reproductive decisionmaking (Human Genetics Commission: Chooosing the future, 2004). The discussion paper summarised information and views so far considered, and was a base for public discussion and comments. Questions asked concern prenatal screening; counselling; risk of disrespect towards disabled owing to option to prevent birth of affected children; and PGD and its indications and choosing characteristics of a donor for a child. People were also encouraged to freely present issues and concerns of ART and genetics (www.hgc.gov.uk).

United Kingdom has not signed the Convention on Human Rights and Biomedicine.

\section{TRANS-ATLANTIC Canada}

Canada's new Assisted Human Reproduction Act (AHRA) of 2004 has been considered as to constitute a successful legislation on human genetic and reproductive technologies. As Canada is said to have a tradition of consensus politics, the new law required hard work and political sophistication. The AHRA will review the law after 3 years experience, which enabled many who were not completely satisfied with the law to support it nonetheless. (Source: the website of the Center for Genetics and Society).

The new law set clear lines between beneficial applications subject to development, and prohibited unacceptable applications of new human genetic and reproductive technologies. The prohibited practices include: The creation of human embryos solely for research; germline engineering (ie, inheritable genetic modification); the creation of human/non-human hybrids and chimeras; all use of somatic cell nuclear transfer (cloning), whether for research or reproduction; sex selection except to prevent, diagnose or treat a sex-linked disorder or disease; and commercial surrogate motherhood contracts and the sale of sperm, eggs and embryos.

Research involving human embryos, including embryonic stem cell research, is permitted using embryos created but not used during in vitro fertilization procedures. The AHRA establishes the Assisted Human Reproduction Agency of Canada (AHRAC) to develop and oversee regulations covering these and other permitted activities. The AHRAC is to license and monitor all private and public fertility clinics, research facilities and other institutions 
whose research or commercial activity involves human gametes or embryos.

USA

There is only one federal statute that aims at the regulation of assisted reproduction: the Fertility Clinic Success Rate and Certification Act of 1992. The purposes of the statute and its related regulations are two-fold: (1) to provide consumers with reliable and useful information about the efficacy of ART services offered by fertility clinics, and (2) to provide states with a model certification process for embryo laboratories. The clinics should give annul reports to the Society for Assisted Reproductive Technology (SART), but no penalties are set for the breach. Unlike the reporting system, adoption of the model programme is entirely voluntary. ${ }^{203}$

There is now no direct federal or state regulation of either PGD or sperm sorting as such. Three federal agencies within the US Department of Health and Human Services oversee areas related to PGD: the Centers for Disease Control and Prevention (CDC), the Food and Drug Administration (FDA) and the Center for Medicare and Medicaid Services (CMS, formerly known as the Health Care Financing Administration).

In 2003, two relevant Bills were introduced in the House of Representatives: a bill as to the Family Building Act (HR $3014 \mathrm{IH}$ ) and the Medicare Infertility Coverage Act (HR 969 $\mathrm{IH})$. However, these were not yet approved in December 2004. The Family Building Act aims to amend the Public Health Service Act and some other statutes in order to require coverage for the treatment of infertility, as the majority of the present group health plans do not provide coverage. One requirement is that the treatment is performed at a medical facility that conforms to the standards of ASRM. Under the Medicare Infertility Coverage Act, the approved technology procedures would be artificial insemination, IVF, embryo transfer, GIFT, intravaginal, intracervical and intrauterine inseminations, any other ART procedure identified by the Secretary as well as services and supplies related to such procedures.

The President's Council on Bioethics (PCBE) has on March 2004 published a report 'Reproduction and responsibility: the regulation of new biotechnologies', which provides with the following information: there are a variety of state laws that bear directly on the clinical practice of assisted reproduction. The vast majority of state statutes directly concerned with assisted reproduction, however, are concerned mostly with the question of access to such services. These states have legislative directives as to whether and to what extent assisted reproduction services will be covered as insurance benefits. Other state statutes regarding assisted reproduction aim to prevent the malfeasance of rogue practitioners (eg, California criminalises unauthorised use of sperm, ova and embryos). Still others focus on the regulation of gamete and embryo donation (eg, California sets forth screening requirements for donated sperm). Many of the state laws dictate parental rights and obligations in the context of assisted reproduction. There are a number of state and federal governmental authorities that do not explicitly aim at the regulation of ART, but indirectly and incidentally provide some measure of oversight and direction.

American Association of Reproductive Medicine (ASRM) is an organisation of close to 9000 physicians, researchers, nurses, technicians and other professionals dedicated to advancing knowledge and expertise in reproductive biology. Affiliated societies include the Society for Assisted Reproductive Technology (SART), The Society for Male Reproduction and Urology, the Society for Reproductive Endocrinology and Infertility, and the Society of Reproductive Surgeons.

The key sources of nongovernmental guidance and oversight for the practice of assisted reproduction are the standards propounded by ASRM, published in conjunction with its sister organization, SART. SART clinics must agree to adhere to these guidelines as a condition of the membership. SART additionally requires certification of its members' embryo labs by the College of American Pathologists, JCAHO, or the New York State Tissue Bank program. Moreover, SART requires its members to comply with the reporting provisions of the federal Fertility Clinic Success Rate and Certification Act. According to SART's website, 95\% of the nation's assisted reproduction clinics are SART members. The practice guidance documents provide direction as to minimal standards for IVF (such as personnel requirements, laboratory requirements, quality assurance and control standards). Specific examples of subjects covered by such documents include guidelines for gamete and embryo donation (ASRM 2002), ICSI, informed consent, induction of ovarian follicle development and ovulation with exogenous gonadotropins, number of embryos transferred and preimplantation genetic diagnosis. Practice committees also evaluate novel procedures. These committees review the existing literature on randomised clinical trials. If two peer-reviewed published studies show that the risk-benefit ratio is acceptable, the procedure is elevated from 'experimental' to 'practice'. ICSI has been elevated to practice status in this way, as have PGD and blastocyst transfer. ASRM 'actively discourages' some procedures on ethical grounds, for example, PGD for elective sex selection, oocyte donation after natural menopause, posthumous reproduction in the absence of advance directives and cloning for reproduction. ${ }^{203}$

ASRM Ethics Committee supports disclosure from parents to their offspring about the use of donor gametes in their conception (Fertility and Sterility 2004; 81: 527-531).

The Hastings Centre's report discusses how new techniques at the intersection of reproductive medicine and genetics raise complex ethical questions that should not be resolved by a largely unregulated market. Rather, they demand 
policies that have been publicly and transparently developed. ${ }^{19}$

\section{RELIGIOUS PERSPECTIVES}

In a pluralistic Europe, it is important to recognise different religious approaches to ART issues. Even the Christian world does not share same opinions. For instance, the Vatican does not approve assisted reproduction, whereas Protestant and Anglican churches accept many forms of ART. $^{250}$

\section{Islamic world}

A Muslim woman is entitled, and even expected, to seek medical fertility treatment and use new technologies in case she is childless. However, third-party assistance shall not be used and therefore donations of germ cells or surrogacy are not allowed in Islam. Embryos can be preserved, but they may be used only for the same couple. The basic concept of Islam is to avoid mixing genes, as Islam enjoins the purity of genes and heredity. It deems that each child should relate to a known father and mother. Multifetal pregnancy reduction is allowed, if it would endanger the pregnancy or if the life or health of the mother is jeopardy. Pregnancy in the postmenopause using donated oocytes is ethically unacceptable and is in general prohibited unless very exceptional reasons.

Islamic opinions vary as to whether elective abortion is allowed or not. In most cases, however, a pregnancy can be terminated if the foetus is found affected before 120 days of pregnancy. ${ }^{251}$ PGD for medical indications is allowed.

A former head of the Al-Azhar Mosque and University, Shaykh Mahmud Shaltut as cited by Abul Fadl Mohsin Ebrahim (1988) released a fatwa (religious decree) that condemns the act of DI and equates it to committing adultery. This fatwa is still in force.

The Fatwas from Al-Azhar, the Islamic Fikh Council in Mecca and the Church of Alexandria issued guidelines, which were adopted by the National Medical Councils and Ministries of Health in the various countries in the 1980s. The guidelines controlled the practices of ART centres and encouraged couples to seek infertility treatment if there was a medical indication. ${ }^{252}$ Recent debates and conference addressed new practices of ART in 2004, which were basically consistent with the previous guidelines

International Islamic Centre for Population Studies and Research, Al-Azhar University, Cairo, organised a workshop in November 2000 to consider use of assisted reproduction technologies (ART) in the Islamic world. The 'Cairo workshop' reinforced a 1997 recommendation that a Standing Committee for Shari's Medical Ethics be constituted to monitor and assess developments in ART practice. Among issues the workshop addressed were equitable access to services for infertile couples of modest means, and regulation of standards of equipment and personnel that ART centres should satisfy to gain approval to offer services. Acceptable uses of PGD were proposed, and follicular maturation research in animals, including in vitro maturation and in vitro growth of oocytes, was encouraged, leading to human applications. Embryo implantation after the death of the husband, postmenopausal pregnancy, uterine transplantation and gene therapy were addressed and human reproductive cloning condemned, but cloning human embryos for stem cell research was considered acceptable. ${ }^{252}$

Israel

Israel has the world's highest per capita rate of IVF clinics. Diverse cultural values affect fertility rates among the population and attitudes towards assisted conception. The duty of procreation, 'be fruitful and multiply', is the first commandment of the Jewish Torah. Many forms of ART are available, ${ }^{253}$ including PGD, even though many rabbinical authorities disapprove the use of donor gametes. In the Jewish Law, interruption of pregnancy is forbidden after the 40th day of pregnancy and therefore the ultraOrthodox community only rarely use the prenatal tests. ${ }^{251}$ PGD is allowed.

\section{CONTRIBUTIONS}

This document was drafted by Sirpa Soini, under the supervision of Professor Helena Kääriäinen. The organising committee and co-authors are the following:

\section{Members of ESHG:}

\section{Anastasiadou, Violetta}

MD, medical geneticist, Head of Clinical Genetics Department

Archibishop Hospital and Cyprus Institute of Neurology and Genetics, Nicosia, Cyprus

\section{Aymé, Ségolène}

MD, medical geneticist and epidemiologist, Project Leader of ORPHANET

INSERM, Paris, France

Braga, Suzanne

MD, medical geneticist, psychoterapist, family therapist and counsellor

SGMG/SSGM, Bern, Switzerland

\section{Cornel, Martina}

MD, PhD, Professor of Community Genetics, VU University Medical Center, Amsterdam, The Netherlands

\section{Coviello, Domenico}

$\mathrm{MD}, \mathrm{PhD}$, medical geneticist, Head of the Laboratory of Medical Genetics

Ospedale Maggiore Policlinico, Mangiagalli e Regina Elena, Milano, Italy

\section{Evers-Kiebooms, Gerry}

Professor, PhD Psychology, Head of the Psychosocial Genetics Unit University Hospital - University of Leuven, Belgium.

\section{Kosztolanyi, György}

Professor, Director of Department of Medical Genetics, University of Pécs, Hungary 


\section{Kääriäinen, Helena}

$\mathrm{MD}, \mathrm{PhD}$, Professor in medical genetics,

Department of Medical genetics, University of Turku, Turku, Finland

\section{Sequeiros, Jorge}

MD, Professor in medical genetics

Head of the Department of Human Genetics, University of Porto, Porto, Portugal

\section{Tranebjaerg, Lisbeth}

$\mathrm{MD}, \mathrm{PhD}$, Professor in genetic audiology

Department of Audiology, H:S Bispebjerg Hospital and Wilhem Johannsen Centre of Functional genomics, University of Kopenhagen, Denmark

\section{Members of ESHRE:}

\section{Geraedts, Joep}

Professor of Genetics and Cell Biology, University Maastricht, Head Department of Clinical Genetics, University Hospital Maastricht, Maastricht, The Netherlands.

Gianaroli, Luca

MD, Specialist in Obstetrics and Gynecology Scientific Director at SISMeR, Centre for Reproductive Medicine, Bologna, Italy

\section{Harper, Joyce}

BSc, PhD, preimplantation genetics

Deputy Director of UCL Centre for PGD, London, UK

\section{Lundin, Kersti}

Associate Professor, Laboratory Director, Reproductive Medicine

SU/Sahlgrenska, Göteborg, Sweden

\section{Sermon, Karen}

MD, Professor in Human and Experimental Genetics, Embryology and Developmental Biology, Medical Genetics and Centres of Medical Genetics and Reproductive Medicine at Brussels Free University, Brussels, Belgium

Two representatives of IPTS:

\section{Ibarreta, Dolores}

$\mathrm{PhD}$, genetics and molecular biology

Scientific officer in the European Commission's Joint Research Center (IPTS)

Seville, Spain

\section{Rodrigues-Cereso, Emilio}

PhD, Agronomist specilised to Molecular Plant Pathology

European Commission's Joint Research Center (IPTS), Seville, Spain

Research assistant:

\section{Soini, Sirpa}

Research assistant, LL.M

University of Turku, Department of Medical Genetics

Turku, Finland

\section{ACKNOWLEDGEMENTS}

The organising committee wishes to thank the following individual experts and institutions for their valuable comments and collaboration during the drafting process of this document and/or in the workshop held in Sevilla during 31 March and 1 April 2005.

\section{Belgium}

- Cassiman, Jean-Jacques. Center for Human Genetics, Leuven.

- DeHooghe, Thomas. Katholic University of Leuven.

- DePaepe, Anna. University of Gent.

- DeRycke, Martine. Centre for Medical Genetics, Brussels.

- Devriendt, Koenraad. Katholic University of Leuven.

- Diercickx, Kris. Katholic University of Leuven.

- Enzlin, Paul. Department of Psychiatry, University Hospital of Leuven.

- Legius, Eric. Katholic University of Leuven.

- Pennings, Guido. University of Gent, Centre for Environmental Philosophy and Bioethics.

- Tournaye, Herman. Dutch-Speaking Brussels Free University, Brussels.

- Welkenhuysen, Myriam. Psychosocial Genetics Unit, Center for Human Genetics, Leuven.

Canada

- Isasi, Rosario. University of Montreal, Centre de recherché en droit public, Quebec.

Check Republic

- Macek, Milan. Institute of Biology and Medical Genetics, University Hospital Motol, Prague.

Denmark

- Bryndorf, Thue. University of Copenhagen, Fertility Clinic.

- Ingerslev, Hans Jakob. Fertility Clinic, Aarhus

- Nyboe Anderssen, Anders. Head of the Fertility Clinic at the Rigshospitalet at Copenhagen University Hospital.

- Rasmussen, Per-Emil. Odense University Hospital, Institute of Clinical Research.

- Schou, Ole. Cryos International Sperm Bank, Aarhus.

Estonia

- Salumets, Andres. Institute of Molecular and Cell Biology, Tartu.

Finland

- Aittomäki, Kristiina. Helsinki University Hospital, Department of Clinical Genetics, Helsinki.

- Burrell, Riitta. Nordic Committee on Bioethics.

- Gissler, Mika. Stakes, Newborn Registry, Helsinki.

- Launis, Veikko. University of Turku.

- Louhiala, Pekka. University of Helsinki.

- Nieminen, Liisa. University of Helsinki.

- Ritvanen, Annukka. Stakes, Finnish Malformation Registry, Helsinki.

- Tiitinen, Aila. Helsinki University Hospital, Infertility Unit, Helsinki.

- Tuuri, Timo. Family Federation, Infertility Clinic, Helsinki. 
France

- Claustres, Mireille. Laboratoire de Génétique Moléculaire et Chromosomique, Institut Universitaire de Recherche Clinique, Montpellier.

- Fournier, Veronique. Hopital Cochin, Centre d'éthique clinique, Paris.

- Pellestor, Franc. IGH, Montpellier.

- Thepot, Francoise. Bureau de la Commission de Génétique de la Fédération Française des CECOS.

- Vekemans, Michel. Hôpital Necker - Enfants maladies, Paris.

- Viville, Stephane. Service de Biologie de la Reproduction, Schiltigheim.

Germany

- Eihenlaub-Rutter, Ursula. Gentechnology/Microbiology, Bielefeld.

- Horsthemke, Bernard. University of Essen, Essen.

- Nippert, Irma. Institute of Human Genetics, University of Münster, Münster.

- Schmidtke, Joerg. Institute of Human Genetics, Hannover.

- Schmutzler Andreas. University of Kiel, Women's Hospital, Kiel.

- Vogt, Peter. Section Molecular Genetics and Infertility, University of Heidelberg, Hedelberg.

- Wieacker, Peter. University of Magdeburg, Magdeburg.

Greece

- Tarlatzis, Basil. Infertility and IVF Center, Thessaloniki.

Hungary

- Mogyorosi, Dorottya. Ministry of Health, Budapest.

Israel

- Shiloh, Shoshana. Department of Psychology, Tel Aviv University, Tel Aviv.

Italy

- Baldi, Marina. Consultorio di Genetica, Rome.

- Costa, Mauro. Department of Genetic, Perinatal and Gynaecological Sciences, Ospedale Galliera, Genova.

- Crosignani, Piergiorgio. University of Milan, Milan.

- Dagna Bricarelli, Franca. Laboratorio di Genetica Humana, EO Ospedale Galliera, Genova.

- Foresta, Carlo. University of Padova, Padova.

- Neri, Demetrio. University of Messina, Reggio Calabria.

- Novelli, Giuseppe. Università degli Studi di Tor Vergata.

The Netherlands

- de Die-Smulders, Christina. Department of Clinical Genetics, University Hospital Maastricht.

- De Wert, Guido. Institute for Bioethics, University of Maastricht, Maastricht.

- Oomens, Marjan. University of Amsterdam.

- Oosterwijk, Cor. EPPOSI delegate, European Genetic Alliances Network, Dutch Genetic Alliance.

- Repping, Sjoerd. Academisch Medisch Centrum, Amsterdam.

- Somsen, Johannes. European University Institute.
Norway

- Hazekamp, Johan T. Volvat Medisinske senter AS, Oslo.

- Kolvik, Rita. Haugesund sjukehus, Helse Fonna

Portugal

- Arriscado, Nunes. Centro de Estudos Sociais, Coimbra.

- Carvalho, Filipa. University of Porto, Porto.

Spain

- Antiñolo, Guillermo. Unidad Clinica de Génetica y Reproducción, Hospital Virgen del Rocio, Seville.

- Borrego Lopez, Salud. Hospitales Universitarios Virgen del Rocio, Seville.

- Egozcue, Jose. Universitat Autonoma de Barcelona, Bellaterra.

- Guillen, Alfredo. Instituto Valenciana de Infertilidad, IVI, Madrid.

- Rocha Castilla, José Luis. Secretario General de Calidad y Modernización, Seville.

- Rubio, Carmen. PGD en IVI Valencia, Madrid.

- Vidal, Francesca. Universitat Autonoma de Barcelona, Bellaterra.

Sweden

- Bergh, Christina. Sahlgrenska University Hospital, Goethenburg.

- Hansson, Charles. Sahlgrenska University Hospital, Goetherburg.

- Hovatta, Outi. Karolinska institutet. Stockholm.

- Kristoffersson, Ulf. Department of Clinical Genetics, University hospital, Lund.

- Wahlstöm, Jan. Department of Clinical Genetics, Sahlgrenska University Hospital, Goethenburg.

Switzerland

- Germond, Mark. Department Obstetrics and Gynecology, University of Lausanne, Lausanne.

- Miny, Peter. Medizinische Genetik UKBB, Basel.

- Reusser, Ruth. EJPD Eidgenössisches Justiz und Polizeidept, Bern.

- Scully, Jackie. University of Basel, Basel.

The United Kingdom

- Braude, Peter. Department of Women's Health, King's College, London.

- Buxton, Jess. Progress Educational Trust and BioNews, London.

- Horsey, Kirsty. Progress Educational Trust and BioNews, London

- Kent, Alistair. GIG.

- Lawford-Davies, James. Epalan Ltd, London.

- Lister, Charles. Human Fertilisation and Embryology Authority, London

- Pembrey, Marcus. Director of Genetics within ALSPAC, Institute of Child Health, Surrey. 
- Richards, Martin. University of Campbridge, Centre for family research, Campbridge.

- Shenfield, Francoise. Elisabeth Garrett Anderson \& Obstetric Hospital, London.

- Tizzard, Juliet. Human Fertilisation and Embryology Authority, London.

- Winston, Robert. Hammersmith Hospital, London.

\section{European Union}

- Fernandez-Zincke, Eduardo. EU Commission, DG Sanco C.

- Forsse, Erik. EU Commission DG RTD

In addition, the organising committee gratefully acknowledges the sponsorship of Abbott to facilitate the organisation of the workshop and the writing of the document.

\section{Glossary and abbreviations}

\section{Glossary and definitions}

ART:

Assisted hatching. In vitro micromanipulation procedure in which the zona pellucida of an embryo (usually at eight-cell stage or a blastocyst) is perforated by chemical, mechanical or laser-assisted methods to assist separation ('hatching') of the blastocyst from the zona pellucida. ${ }^{5}$

CGH: Comparative genome hybridisation, an analysing method to detect genomic imbalances.

Cryopreservation: Freezing the gametes or the embryo has helped to make the IVF treatment more flexible. However, in particular, oocytes suffer from this procedure. All stages of an embryo from the zygote (two pronucleate) stage to the blastocyst stage can be cryopreserved.
PGS:

Fluorescence in situ hybridisation, an analysing method to detect chromosomal anomalies.

GIFT:

HLA:

ICSI:

IVF:

Gamete intrafallopian transfer. Both types of gametes (sperm and oocytes) are transferred to the fallopian tubes.

Human leukocyte antigen.

Intracytoplasmic sperm injection. Method to assist fertilisation, variant of IVF. A single spermatozoon is injected through the zona pellucida into the ooplasm of the oocyte. ICSI is indicated in cases of severe male-factor infertility, in which male patients have either malformed sperm or an abnormally low sperm count, and in cases of previous failed fertilisation with conventional IVF.

In vitro fertilization. Method used to assist fertilisation and help a couple achieve a pregnancy. The woman is superovulated to produce multiple oocytes, which are collected and mixed with sperm. After fertilisation, the oocytes are kept in culture. Usually, one to two resulting embryos are transferred to the uterus between days 2 and 5 of development. Indications include blocked fallopian tubes, infertility of unknown cause, etc.

MESA:

Microscopic epididymal sperm aspiration, a procedure in which spermatozoa is obtained from the epididymis by surgical excision.

PCR:

Polymerase chain reaction (a method that can be used in PGD for analysing genes in cases of monogenic diseases).

PESA:

Percutaneous epididymal sperm aspiration, where sperm are aspirated from the epididymis using needle biopsy.

PGD: $\quad$ Preimplantation genetic diagnosis. Method used to determine a specific abnormality in embryos generated by parents carrying that abnormality. Indications include patients carrying X-linked disease, monogenic disorders and chromosomal abnormalities such as translocations, etc. Preimplantation genetic screening (for aneuploidy). Method used to 
PND:

SET:

TESA:

TESE:

TVOD:

ZIFT:

ZP:

\section{Institutions and organisations and their websites}

ASRM:

American Society for Reproductive Medicine, www.asrm.org
CCNE: normal embryo for transfer in an IVF/ICSI cycle. Usually, between five and nine chromosomes are examined.

Prenatal diagnosis is a (molecular, cytogenetic, biochemical) diagnostic test carried out on a developing foetus after amniocentesis, chorionic villus sampling or foetal blood sampling.

Single embryo transfer (eSET elective SET).

Testicular sperm aspiration. Spermatozoa is obtained directly from the testicle, by needle aspiration of testicular tissue.

Testicular sperm extraction, where spermatozoa is obtained from the testicle by use of surgical excision. Transvaginal ovarian drilling, a surgical treatment for polycystic ovary syndrome to improve the clinical outcome in ART.

Zygote intrafallopian transfer. The zygote is transferred into the fallopian tube in its pronuclear stage of development. In ZIFT, the embryo is placed (via laparoscopy) directly into the fallopian tube, rather than into the uterus. In this way, it is similar to the transfer of gametes in GIFT. Some opt for ZIFT on the theory that it enhances the likelihood of implantation, given that the embryo matures on its way to the uterus, presumably as it would in natural conception and implantation. Additionally, many patients prefer ZIFT to GIFT because the process of fertilization and early development of the embryo may be monitored.

Zona pellucida.

WMA:

UNESCO:

GIG:

HGC:

HFEA:

IBC
Comité counsultatif national d'éthique pour les sciences de la vie et de la santé, www.ccne-ethique.fr

CIOMS: Council for International Organizations of Medical Sciences, www.cioms.ch

COE: Council of Europe, www.coe.int

EDDNAL: $\quad$ European Directory of DNA Diagnostic Laboratories, www.eddnal.com

EGE: $\quad$ European Group on Ethics in Science and New Developments, http://europa.eu.int/ comm/european_group_ethics/index_en. htm

EMQN: $\quad$ European Molecular Genetics Quality European Society of Human Genetics www.eshg.org

ESHRE: $\quad$ European Society of Human Reproduction and Embryology www.eshre.com

Ethikrat: German National Ethics Council, www.ethikat.org

EUROCAT: European Registration of Congenital Anomalies, www.eurocat.ulster.ac.uk

EURORDIS: European Organisation of Rare Diseases, www.eurordis.com

ICBDSR: International Clearinghouse for Birth Defects Research and Surveillance, www.icbd.org

IFFS: International Federation of Fertility Societies, www.iffs-reproduction.org Institute for Prospective Technological Studies, www.jrc.es

Joint Research Center, www.jcr.coe.eu.int President's Council on Bioethics, www.bioethics.gov

Preimplantation Genetic Diagnosis International Society, www.pgdis.org

World Health Organisation, www.who. int

World Medical Association, www.wma. net

United Nations Educational, Scientific and Cultural Organisation, www.portal. unesco.org 